\title{
The two-nucleon system at next-to-next-to-next-to-leading order ${ }^{\text {is }}$
}

\author{
E. Epelbaum ${ }^{\mathrm{a}, *}$, W. Glöckle ${ }^{\mathrm{b}}$, Ulf-G. Meißner ${ }^{\mathrm{c}, \mathrm{d}}$ \\ a Jefferson Laboratory, Theory Division, Newport News, VA 23606, USA \\ ${ }^{\mathrm{b}}$ Ruhr-Universität Bochum, Institut für Theoretische Physik II, D-44870 Bochum, Germany \\ ${ }^{\mathrm{c}}$ Universität Bonn, Helmholtz-Institut für Strahlen- und Kernphysik (Theorie), D-53115 Bonn, Germany \\ ${ }^{\mathrm{d}}$ Forschungszentrum Jülich, Institut für Kernphysik (Theorie), D-52425 Jülich, Germany
}

Received 19 May 2004; received in revised form 7 September 2004; accepted 30 September 2004

Available online 26 October 2004

\begin{abstract}
We consider the two-nucleon system at next-to-next-to-next-to-leading order $\left(\mathrm{N}^{3} \mathrm{LO}\right)$ in chiral effective field theory. The two-nucleon potential at $\mathrm{N}^{3} \mathrm{LO}$ consists of one-, two- and three-pion exchanges and a set of contact interactions with zero, two and four derivatives. In addition, one has to take into account various isospin-breaking and relativistic corrections. We employ spectral function regularization for the multi-pion exchanges. Within this framework, it is shown that the three-pion exchange contribution is negligibly small. The low-energy constants (LECs) related to pion-nucleon vertices are taken consistently from studies of pion-nucleon scattering in chiral perturbation theory. The total of 26 four-nucleon LECs has been determined by a combined fit to some $n p$ and $p p$ phase shifts from the Nijmegen analysis together with the $n n$ scattering length. The description of nucleonnucleon scattering and the deuteron observables at $\mathrm{N}^{3} \mathrm{LO}$ is improved compared to the one at NLO and NNLO. The theoretical uncertainties in observables are estimated based on the variation of the cut-offs in the spectral function representation of the potential and in the regulator utilized in the Lippmann-Schwinger equation.
\end{abstract}

(C) 2004 Elsevier B.V. All rights reserved.

\footnotetext{
Wh Work supported in part by US Department of Energy under contract number DE-AC05-84ER40150.

* Corresponding author.

E-mail addresses: epelbaum@jlab.org (E. Epelbaum), walter.gloeckle@tp2.ruhr-uni-bochum.de (W. Glöckle), meissner@itkp.uni-bonn.de (U.-G. Meißner).
} 


\section{Introduction}

Since the seminal work of Weinberg [1] to derive the forces between two, three, ... nucleons from chiral effective field theory, there has been a flurry of activities to work out the consequences of such an approach, to improve that scheme or to construct alternatives, for reviews see $[2,3]$. Here, we will be dealing with a modified Weinberg scheme, in which pions are treated nonperturbatively and the power counting is applied to the nucleon-nucleon potential. The potential consists of one-, two- , ... pion-exchanges (1PE, 2PE, ...) and a string of contact interactions with an increasing number of derivatives (zero, two, four, ...) that parameterize the shorter ranged components of the nuclear force (the precise framework is specified in more detail below). Such an approach has a variety of advantages over more conventional schemes or phenomenological models. First, it offers a systematic method to improve calculations by going to ever increasing orders in the power counting and it allows to give theoretical uncertainties. Second, one can consistently derive twoand three-nucleon forces (see, e.g., [4]), which has never been achieved before and paves, e.g., the way for a new look at the problem of nuclear matter. Third, nucleon and nuclear properties can be calculated from one effective Lagrangian, which is of particular importance if one intends to extract neutron properties from (electromagnetically induced) measurements on light nuclei in a controlled theoretical way. In this paper, we present the nucleon-nucleon potential at next-to-next-to-next-to-leading order $\left(\mathrm{N}^{3} \mathrm{LO}\right)$ in the chiral expansion, extending our earlier work, and we apply this potential to observables in two-nucleon systems. Our work differs from the one of Entem and Machleidt (EM), who first presented an $\mathrm{N}^{3} \mathrm{LO}$ potential in Ref. [5], in various ways. First, they use the two-pion exchange contributions based on dimensional regularization, which have a very singular short-range behavior. We employ spectral function regularization, which allows for a better separation between the long- and short-distance contributions. Second, EM present results only for one choice of the cut-off necessary to regulate the high-momentum components in the Lippmann-Schwinger equation to generate the scattering and the bound states. ${ }^{1}$ We perform systematic variations of this cut-off and other parameters which allows us to give not only central values but also theoretical uncertainties. Third, our treatment of the isospin breaking effects differs from the one of EM (which is based on our earlier work [7]). Fourth, we employ a relativistic version of the Schrödinger equation, which allows to calculate consistently relativistic corrections also in three and four nucleon systems. Other less significant differences will be discussed in due course. We believe that with the material presented here an important step has been made to put precision calculations in nuclear physics on a firm theoretical basis, which not only allows to readdress many issues that have already been investigated in quite a detail but will also open new areas of testing chiral dynamics in few-nucleon systems or shed more light on the issue of the nuclear forces in the limit of vanishing quark masses (see [8-11] for earlier work on that topic).

Our manuscript is organized as follows. In Section 2, we explicitly give the potential at $\mathrm{N}^{3}$ LO. The contributions up to NNLO have already been extensively discussed in [12-17]. The $\mathrm{N}^{3} \mathrm{LO}$ corrections due to two- and three-pion exchange have been derived recently in

\footnotetext{
1 The results for two different choices of the cut-off in the Lippmann-Schwinger equation are shown in [6].
} 
[18-20] using dimensional regularization to regularize divergent loop integrals. Throughout this work, we use a different regularization scheme for the potential, namely the spectral function regularization (SFR). This approach has been recently proposed and successfully applied at NLO and NNLO [17,21]. As demonstrated in these references, the SFR scheme allows to significantly improve the convergence of chiral effective field theory (EFT) for the two-nucleon system. We also give an overview of various isospin-breaking interactions including electromagnetic forces and discuss the regularization procedure necessary to render the (iterated) potential finite. In Section 3 we deal with the scattering equation. In order to account for the relativistic corrections to the nucleon kinetic energy, we have decided to use the Lippmann-Schwinger equation with the relativistic expression for the kinetic energy. Such an approach can naturally be extended to few-nucleon systems and to processes with external probes. We also discuss how to cast the relativistic Lippmann-Schwinger equation into a nonrelativistic form, which might be useful in certain applications. Various deuteron properties are considered in Section 4 using both relativistic and nonrelativistic Schrödinger equations. The fitting procedure to determine the low-energy constants (LECs) and the accuracy of the fits are detailed in Section 5. Results for phase shifts and the deuteron (bound state) properties are displayed and discussed in Section 6. Our findings are summarized in Section 7. The appendices contain details on the kinematics, the partial wave decomposition, the momentum space treatment of the Coulomb interaction and the effective range expansion.

\section{The two-nucleon potential at $\mathbf{N}^{3} \mathrm{LO}$}

\subsection{General remarks}

Before going into details of calculations, we would like to make certain general remarks. As already pointed out in the introduction, we strictly follow the scheme suggested by Weinberg [1]. In this approach one uses the EFT technique to derive nuclear forces from the most general (approximately) chiral invariant effective Lagrangian. The $N N S$-matrix is obtained via (nonperturbative) solution of the Lippmann-Schwinger (LS) equation. In most practical calculations (including the present one), the later step can only be performed numerically.

Starting from the most general chiral invariant effective Hamiltonian density for pions and nucleons one can derive energy-independent and hermitian nuclear forces by a variety of methods including the method of unitary transformation, see, e.g., [10]. The resulting nucleonic forces are ordered by the power of the generic low-momentum scale $Q$ related to the three-momenta of nucleons, the pion mass and typical four-momenta of virtual pions:

$$
V \sim \mathcal{O}\left[\frac{1}{F_{\pi}^{2}}\left(\frac{Q}{\Lambda_{\chi}}\right)^{\nu}\right],
$$

where $F_{\pi}$ is the pion decay constant and $\Lambda_{\chi}$ is the chiral symmetry breaking scale or, more generally, the smaller of the chiral symmetry breaking scale and the scale $\Lambda_{\text {LEC }}$ associated 
with the LECs accompanying four-nucleon contact interactions (as discussed below). The power $v$ for a given diagram can be calculated using the rules of dimensional analysis [1]

$$
v=-2+2 E_{n}+2(L-C)+\sum_{i} V_{i} \Delta_{i},
$$

where $E_{n}, L, C$ and $V_{i}$ are the numbers of nucleons, loops, separately connected pieces and vertices of type $i$, respectively. Further, the quantity $\Delta_{i}$, which defines the dimension of a vertex of type $i$, is given by

$$
\Delta_{i}=d_{i}+\frac{1}{2} n_{i}-2
$$

with $d_{i}$ the number of derivatives or $M_{\pi}$ insertions and $n_{i}$ the number of nucleon lines at the vertex $i$. One has $\Delta_{i} \geqslant 0$ as a consequence of chiral invariance. This leads to $v \geqslant 0$ for connected diagrams with two and more nucleons. One also recognizes that the graphs with loops are suppressed and that $(n+1)$-nucleon forces appear at higher orders than the $n$-nucleon ones. We note, however, that the formula (2.2) does not apply to a specific sort of diagrams, sometimes referred to as reducible, whose contributions are enhanced due to the presence of anomalously small energy denominators resulting from purely nucleonic intermediate states. Such reducible diagrams are responsible for the nonperturbative aspect in the few-nucleon problem and must be summed up to infinite order. They, however, do not contribute to the nuclear potential and result from iteration of the potential in the Lippmann-Schwinger equation.

It remains to specify our way of counting the nucleon mass. In the single-nucleon sector it appears to be natural to treat the nucleon mass $m$ in the same way as the chiral symmetry breaking scale $\Lambda_{\chi} \sim 1 \mathrm{GeV}$. As argued in [1], in the few-nucleon sector consistency requires that the nucleon mass is considered as a much larger scale compared to the chiral symmetry breaking scale. If one adopts the counting rule $m \sim \Lambda_{\chi}$, no nonperturbative resummation of the amplitude is required from the point of view of the chiral power counting. In this work we adopt the counting rule $m / Q \sim\left(\Lambda_{\chi} / Q\right)^{2}$, which has also been used in [12].

In the following sections we will discuss various contributions to the NN potential up to $\mathrm{N}^{3} \mathrm{LO}$ including isospin-breaking corrections.

\subsection{Contact terms}

We consider first the contact terms of the two-nucleon potential. To the accuracy we are working, the potential in the center-of-mass system (cms) for initial and final nucleon momenta $\vec{p}$ and $\vec{p}^{\prime}$, respectively, takes the form: ${ }^{2}$

$$
\begin{aligned}
& V_{\text {cont }}=V_{\text {cont }}^{(0)}+V_{\text {cont }}^{(2)}+V_{\text {cont }}^{(4)}, \\
& V_{\text {cont }}^{(0)}=C_{S}+C_{T} \vec{\sigma}_{1} \cdot \vec{\sigma}_{2},
\end{aligned}
$$

$\overline{2}$ We use the notation of Ref. [16] (Ref. [6]) for $V^{(0)}$ and $V^{(2)}\left(V^{(4)}\right)$. 


$$
\begin{aligned}
V_{\mathrm{cont}}^{(2)}= & C_{1} \vec{q}^{2}+C_{2} \vec{k}^{2}+\left(C_{3} \vec{q}^{2}+C_{4} \vec{k}^{2}\right)\left(\vec{\sigma}_{1} \cdot \vec{\sigma}_{2}\right)+i C_{5} \frac{1}{2}\left(\vec{\sigma}_{1}+\vec{\sigma}_{2}\right) \cdot(\vec{q} \times \vec{k}) \\
& +C_{6}\left(\vec{q} \cdot \vec{\sigma}_{1}\right)\left(\vec{q} \cdot \vec{\sigma}_{2}\right)+C_{7}\left(\vec{k} \cdot \vec{\sigma}_{1}\right)\left(\vec{k} \cdot \vec{\sigma}_{2}\right), \\
V_{\mathrm{cont}}^{(4)}= & D_{1} \vec{q}^{4}+D_{2} \vec{k}^{4}+D_{3} \vec{q}^{2} \vec{k}^{2}+D_{4}(\vec{q} \times \vec{k})^{2} \\
& +\left(D_{5} \vec{q}^{4}+D_{6} \vec{k}^{4}+D_{7} \vec{q}^{2} \vec{k}^{2}+D_{8}(\vec{q} \times \vec{k})^{2}\right)\left(\vec{\sigma}_{1} \cdot \vec{\sigma}_{2}\right) \\
& +i\left(D_{9} \vec{q}^{2}+D_{10} \vec{k}^{2}\right) \frac{\vec{\sigma}_{1}+\vec{\sigma}_{2}}{2} \cdot(\vec{q} \times \vec{k}) \\
& +\left(D_{11} \vec{q}^{2}+D_{12} \vec{k}^{2}\right)\left(\vec{\sigma}_{1} \cdot \vec{q}\right)\left(\vec{\sigma}_{2} \cdot \vec{q}\right)+\left(D_{13} \vec{q}^{2}+D_{14} \vec{k}^{2}\right)\left(\vec{\sigma}_{1} \cdot \vec{k}\right)\left(\overrightarrow{\sigma_{2}} \cdot \vec{k}\right) \\
& +D_{15}\left(\vec{\sigma}_{1} \cdot(\vec{q} \times \vec{k}) \vec{\sigma}_{2} \cdot(\vec{q} \times \vec{k})\right)
\end{aligned}
$$

with $\vec{q}=\vec{p}^{\prime}-\vec{p}$ and $\vec{k}=\left(\vec{p}+\vec{p}^{\prime}\right) / 2$. The superscripts denote the corresponding chiral order as defined in Eq. (2.2). Notice that the contact operator basis in Eq. (2.4) represents just one particular choice among many others. One could equally well use another set of 24 independent contact operators including for instance terms which contain the product of isospin matrices $\vec{\tau}_{1} \cdot \vec{\tau}_{2}$. A one-to-one correspondence between different sets of contact operators can be established upon performing antisymmetrization of the potential, see Ref. [22] for more details. Notice that we have only shown isospin-invariant terms in Eq. (2.4). Isospin-breaking short-range corrections will be specified below.

The terms in Eq. (2.4) feed into the matrix-elements of the two $S$-waves $\left({ }^{1} S_{0},{ }^{3} S_{1}\right)$, the four $P$-waves $\left({ }^{1} P_{1},{ }^{3} P_{1},{ }^{3} P_{2},{ }^{3} P_{0}\right)$, the four $D$-waves $\left({ }^{1} D_{2},{ }^{3} D_{2},{ }^{3} D_{3},{ }^{3} D_{1}\right)$ and the two lowest transition potentials $\left({ }^{3} D_{1}-{ }^{3} S_{1},{ }^{3} F_{2}-{ }^{3} P_{2}\right)$ in the following way:

$$
\begin{aligned}
& \left\langle{ }^{1} S_{0}\left|V_{\text {cont }}\right|{ }^{1} S_{0}\right\rangle=\tilde{C}_{{ }^{S_{0}}}+C_{{ }^{1} S_{0}}\left(p^{2}+p^{\prime 2}\right)+D_{{ }_{1} S_{0}}^{1} p^{2} p^{\prime 2}+D_{{ }_{1} S_{0}}^{2}\left(p^{4}+p^{\prime 4}\right) \text {, } \\
& \left\langle{ }^{3} S_{1}\left|V_{\text {cont }}\right|{ }^{3} S_{1}\right\rangle=\tilde{C}_{{ }^{3} S_{1}}+C_{{ }^{3} S_{1}}\left(p^{2}+p^{\prime 2}\right)+D_{{ }^{3} S_{1}}^{1} p^{2} p^{\prime 2}+D_{{ }^{2} S_{1}}^{2}\left(p^{4}+p^{\prime 4}\right) \text {, } \\
& \left\langle{ }^{1} P_{1}\left|V_{\text {cont }}\right|{ }^{1} P_{1}\right\rangle=C^{{ }_{1} P_{1}} p p^{\prime}+D^{{ }^{1} P_{1}} p p^{\prime}\left(p^{2}+p^{\prime 2}\right), \\
& \left\langle{ }^{3} P_{1}\left|V_{\text {cont }}\right|^{3} P_{1}\right\rangle=C_{3} P_{1} p p^{\prime}+D_{3} P_{1} p p^{\prime}\left(p^{2}+p^{\prime 2}\right), \\
& \left\langle{ }^{3} P_{0}\left|V_{\text {cont }}\right|^{3} P_{0}\right\rangle=C^{3} P_{0} p p^{\prime}+D^{3} P_{0} p p^{\prime}\left(p^{2}+p^{\prime 2}\right), \\
& \left\langle{ }^{3} P_{2}\left|V_{\text {cont }}\right|{ }^{3} P_{2}\right\rangle=C_{3} P_{2} p p^{\prime}+D^{3} P_{2} p p^{\prime}\left(p^{2}+p^{\prime 2}\right), \\
& \left\langle{ }^{1} D_{2}\left|V_{\text {cont }}\right|{ }^{1} D_{2}\right\rangle=D^{1} D_{2} p^{2} p^{\prime 2} \text {, } \\
& \left\langle{ }^{3} D_{2}\left|V_{\text {cont }}\right|{ }^{3} D_{2}\right\rangle=D^{3} D_{2} p^{2} p^{\prime 2} \text {, } \\
& \left\langle{ }^{3} D_{1}\left|V_{\text {cont }}\right|{ }^{3} D_{1}\right\rangle=D^{3} D_{1} p^{2} p^{\prime 2} \text {, } \\
& \left\langle{ }^{3} D_{3}\left|V_{\text {cont }}\right|{ }^{3} D_{3}\right\rangle=D^{3} D_{3} p^{2} p^{\prime 2} \text {, }
\end{aligned}
$$

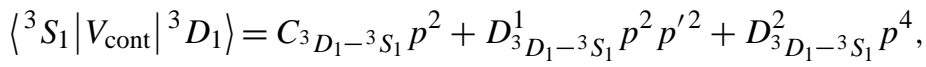

$$
\begin{aligned}
& \left\langle{ }^{3} D_{1}\left|V_{\text {cont }}\right|{ }^{3} S_{1}\right\rangle=C^{3} D_{1}-{ }^{3} S_{1} p^{\prime 2}+D^{1}{ }^{1} D_{1}-{ }^{3} S_{1} p^{2} p^{\prime 2}+D_{3}^{2} D_{1}-{ }^{3} S_{1} p^{\prime 4} \text {, } \\
& \left\langle{ }^{3} P_{2}\left|V_{\text {cont }}\right|{ }^{3} F_{2}\right\rangle=D^{3} F_{2}-{ }^{3} P_{2} p^{3} p^{\prime} \text {, } \\
& \left\langle{ }^{3} F_{2}\left|V_{\text {cont }}\right|{ }^{3} P_{2}\right\rangle=D^{3} F_{2}-{ }^{3} P_{2} p p^{\prime 3},
\end{aligned}
$$

with $p=|\vec{p}|$ and $p^{\prime}=\left|\vec{p}^{\prime}\right|$. The spectroscopic LECs are related to the ones in Eq. (2.4) according to the following relations: 


$$
\begin{aligned}
& \tilde{C}_{{ }^{S} S_{0}}=4 \pi\left(C_{S}-3 C_{T}\right), \\
& \tilde{C}_{{ } S_{1}}=4 \pi\left(C_{S}+C_{T}\right) \text {, } \\
& C^{{ }^{1} S_{0}}=\pi\left(4 C_{1}+C_{2}-12 C_{3}-3 C_{4}-4 C_{6}-C_{7}\right) \text {, } \\
& C_{3 S_{1}}=\frac{\pi}{3}\left(12 C_{1}+3 C_{2}+12 C_{3}+3 C_{4}+4 C_{6}+C_{7}\right) \text {, } \\
& C^{{ } P_{1}}=\frac{2 \pi}{3}\left(-4 C_{1}+C_{2}+12 C_{3}-3 C_{4}+4 C_{6}-C_{7}\right), \\
& { }^{C_{3} P_{1}}=\frac{2 \pi}{3}\left(-4 C_{1}+C_{2}-4 C_{3}+C_{4}+2 C_{5}-8 C_{6}+2 C_{7}\right) \\
& C_{3 P_{2}}=\frac{2 \pi}{3}\left(-4 C_{1}+C_{2}-4 C_{3}+C_{4}-2 C_{5}\right), \\
& { }^{C_{3} P_{0}}=\frac{2 \pi}{3}\left(-4 C_{1}+C_{2}-4 C_{3}+C_{4}+4 C_{5}+12 C_{6}-3 C_{7}\right) \text {, } \\
& C^{C_{D_{1}}-{ }^{3} S_{1}}=C_{\epsilon 1}=\frac{2 \sqrt{2} \pi}{3}\left(4 C_{6}+C_{7}\right), \\
& D_{1_{0}}^{1}=\frac{\pi}{6}\left(80 D_{1}+5 D_{2}+4 D_{3}+16 D_{4}-240 D_{5}-15 D_{6}-12 D_{7}-48 D_{8}\right. \\
& \left.-80 D_{11}-4 D_{12}-4 D_{13}-5 D_{14}-16 D_{15}\right) \text {, } \\
& D_{S_{0}}^{2}=\frac{\pi}{4}\left(16 D_{1}+D_{2}+4 D_{3}-48 D_{5}-3 D_{6}-12 D_{7}-16 D_{11}-4 D_{12}\right. \\
& \left.-4 D_{13}-D_{14}\right) \\
& D_{{ }^{3} S_{1}}^{1}=\frac{\pi}{18}\left(240 D_{1}+15 D_{2}+12 D_{3}+48 D_{4}+240 D_{5}+15 D_{6}+12 D_{7}+48 D_{8}\right. \\
& \left.+80 D_{11}+4 D_{12}+4 D_{13}+5 D_{14}+16 D_{15}\right) \text {, } \\
& D_{3 S_{1}}^{2}=\frac{\pi}{12}\left(48 D_{1}+3 D_{2}+12 D_{3}+48 D_{5}+3 D_{6}+12 D_{7}+16 D_{11}+4 D_{12}\right. \\
& \left.+4 D_{13}+D_{14}\right) \\
& { }^{D^{1} P_{1}}=-\frac{\pi}{3}\left(16 D_{1}-D_{2}-48 D_{5}+3 D_{6}-16 D_{11}+D_{14}\right) \text {, } \\
& D^{3} P_{1}=-\frac{\pi}{6}\left(32 D_{1}-2 D_{2}+32 D_{5}-2 D_{6}-8 D_{9}-2 D_{10}+48 D_{11}+4 D_{12}\right. \\
& \left.-4 D_{13}-3 D_{14}\right) \\
& { }^{D_{3} P_{2}}=-\frac{\pi}{30}\left(160 D_{1}-10 D_{2}+160 D_{5}-10 D_{6}+40 D_{9}+10 D_{10}+16 D_{11}\right. \\
& \left.-4 D_{12}+4 D_{13}-D_{14}\right) \\
& { }^{D_{3} P_{0}}=-\frac{\pi}{3}\left(16 D_{1}-D_{2}+16 D_{5}-D_{6}-8 D_{9}-2 D_{10}-32 D_{11}-4 D_{12}\right. \\
& \left.+4 D_{13}+2 D_{14}\right) \\
& { }^{D_{1} D_{2}}=\frac{2 \pi}{15}\left(16 D_{1}+D_{2}-4 D_{3}-4 D_{4}-48 D_{5}-3 D_{6}+12 D_{7}+12 D_{8}\right. \\
& \left.-16 D_{11}+4 D_{12}+4 D_{13}-D_{14}+4 D_{15}\right) \text {, } \\
& { }^{D_{3} D_{2}}=\frac{\pi}{15}\left(32 D_{1}+2 D_{2}-8 D_{3}-8 D_{4}+32 D_{5}+2 D_{6}-8 D_{7}-8 D_{8}-8 D_{9}\right. \\
& \left.+2 D_{10}+48 D_{11}-12 D_{12}-12 D_{13}+3 D_{14}+16 D_{15}\right) \text {, }
\end{aligned}
$$




$$
\begin{aligned}
& D^{{ }^{3} D_{1}}= \frac{\pi}{45}\left(96 D_{1}+6 D_{2}-24 D_{3}-24 D_{4}+96 D_{5}+6 D_{6}-24 D_{7}-24 D_{8}\right. \\
&\left.-72 D_{9}+18 D_{10}-80 D_{11}+20 D_{12}+20 D_{13}-5 D_{14}-64 D_{15}\right), \\
& D^{{ }_{3} D_{3}}= \frac{2 \pi}{15}\left(16 D_{1}+D_{2}-4 D_{3}-4 D_{4}+16 D_{5}+D_{6}-4 D_{7}-4 D_{8}+8 D_{9}\right. \\
&\left.-2 D_{10}-4 D_{15}\right), \\
& D_{{ }^{3} D_{1}-{ }^{3} S_{1}}^{1}=D_{\epsilon 1}^{1}=\frac{\sqrt{2} \pi}{18}\left(112 D_{11}-4 D_{12}-4 D_{13}+7 D_{14}-16 D_{15}\right), \\
& D_{{ }^{2} D_{1}-{ }^{3} S_{1}}^{2}=D_{\epsilon 1}^{2}=\frac{\sqrt{2} \pi}{6}\left(16 D_{11}+4 D_{12}+4 D_{13}+D_{14}\right), \\
& D_{3{ }_{F}-{ }^{3} P_{2}}=D_{\epsilon 2}=-\frac{\sqrt{6} \pi}{15}\left(16 D_{11}-4 D_{12}+4 D_{13}-D_{14}\right) .
\end{aligned}
$$

These 24 constants are not fixed by chiral symmetry and have to be determined by a fit to data or phase shifts and mixing parameters in the corresponding channels. From each of the two $S$-waves, we can determine four parameters, whereas each of the four $P$-waves and the mixing parameter $\epsilon_{1}$ contain two free parameters. Further, one free parameter contributes to each of the four $D$-waves and to the mixing parameter $\epsilon_{2}$. Of course, we have to account for the channel coupling in the mixed spin-triplet partial waves. Once the spectroscopic LECs have been determined, the original $C_{S}, C_{T}, C_{1}, \ldots, C_{7}$ and $D_{1}, \ldots, D_{15}$ are fixed uniquely.

\subsection{One-, two- and three-pion exchange}

Consider now one-, two- and three-pion exchange (3PE) contributions $V_{1 \pi}, V_{2 \pi}$ and $V_{3 \pi}$, respectively. At $\mathrm{N}^{3} \mathrm{LO}\left(Q^{4}\right)$ in the low-momentum expansion $Q$ they can be written as

$$
\begin{aligned}
& V_{1 \pi}=V_{1 \pi}^{(0)}+V_{1 \pi}^{(2)}+V_{1 \pi}^{(3)}+V_{1 \pi}^{(4)}+\cdots, \\
& V_{2 \pi}=V_{2 \pi}^{(2)}+V_{2 \pi}^{(3)}+V_{2 \pi}^{(4)}+\cdots, \\
& V_{3 \pi}=V_{3 \pi}^{(4)}+\cdots
\end{aligned}
$$

Here the superscripts denote the corresponding chiral order and the ellipses refer to $Q^{5}$ and higher order terms which are not considered in the present work. Contributions due to exchange of four and more pions are further suppressed: $n$-pion exchange diagrams start to contribute at the order $Q^{2 n-2}$, see, e.g., [1].

In the following we will give explicit expressions for the individual contributions in Eq. (2.7). The pion-exchange $N N$ potential in the two-nucleon center-of-mass system (c.m.s) takes the form:

$$
\begin{aligned}
V= & V_{C}+\boldsymbol{\tau}_{1} \cdot \boldsymbol{\tau}_{2} W_{C}+\left[V_{S}+\boldsymbol{\tau}_{1} \cdot \boldsymbol{\tau}_{2} W_{S}\right] \vec{\sigma}_{1} \cdot \vec{\sigma}_{2}+\left[V_{T}+\boldsymbol{\tau}_{1} \cdot \boldsymbol{\tau}_{2} W_{T}\right] \vec{\sigma}_{1} \cdot \vec{q} \vec{\sigma}_{2} \cdot \vec{q} \\
& +\left[V_{L S}+\boldsymbol{\tau}_{1} \cdot \boldsymbol{\tau}_{2} W_{L S}\right] i\left(\vec{\sigma}_{1}+\vec{\sigma}_{2}\right) \cdot(\vec{q} \times \vec{k}) \\
& +\left[V_{\sigma L}+\boldsymbol{\tau}_{1} \cdot \boldsymbol{\tau}_{2} W_{\sigma L}\right] \vec{\sigma}_{1} \cdot(\vec{q} \times \vec{k}) \vec{\sigma}_{2} \cdot(\vec{q} \times \vec{k}),
\end{aligned}
$$


where the superscripts $C, S, T, L S$ and $\sigma L$ of the scalar functions $V_{C}, \ldots, W_{\sigma L}$ refer to central, spin-spin, tensor, spin-orbit and quadratic spin-orbit components, respectively.

The leading order 1PE potential is given by

$$
W_{T}^{(0)}(q)=-\left(\frac{g_{A}}{2 F_{\pi}}\right)^{2} \frac{\vec{\sigma}_{1} \cdot \vec{q} \vec{\sigma}_{2} \cdot \vec{q}}{q^{2}+M_{\pi}^{2}} .
$$

At NLO one has to take into account various corrections which result from one-loop diagrams with the leading vertices and tree graphs with one insertion of the $d_{16^{-}}$and $\tilde{d}_{28}$-vertices (in the notation of Ref. [23]) from the dimension three Lagrangian $\mathcal{L}_{\pi N}$ and $l_{3,4}$-vertices from the dimension four Lagrangian $\mathcal{L}_{\pi}$. All these graphs lead just to renormalization of the LECs $g_{A}, F_{\pi}$ and the pion mass $M_{\pi}$, see [10] for more details. In addition, one has a contribution from the 1PE graphs with one $g_{A}$-vertex replaced by the $d_{18}$-vertex from the dimension three $\pi N$ Lagrangian. This correction leads to the so-called Goldberger-Treiman discrepancy and can be accounted for by the replacement

$$
g_{A} \rightarrow g_{A}-2 d_{18} M_{\pi}^{2}
$$

in Eq. (2.9). The corrections at NNLO arise from one-loop diagrams with one subleading $\pi N N$ vertex and lead to renormalization of the LEC $g_{A}$ [14]. The corrections to 1PE at $\mathrm{N}^{3} \mathrm{LO}$ are due to two-loop diagrams with all vertices of the lowest chiral dimension, one-loop graphs with one subleading vertex from $\mathcal{L}_{\pi N}$ or $\mathcal{L}_{\pi}$ and tree graphs with two subleading vertices or one sub-subleading vertex. After performing renormalization of the LECs, one finds the $\mathrm{N}^{3} \mathrm{LO}$ contribution to the 1PE potential to be proportional to [24]

$$
W_{T}^{(4)}(q) \propto \frac{1}{q^{2}+M_{\pi}^{2}} F\left(q^{2}\right),
$$

where the function $F\left(q^{2}\right)=\alpha_{1} M_{\pi}^{4}+\alpha_{2} M_{\pi}^{2} q^{2}+\alpha_{3} q^{4}$ can be viewed as the pion-nucleon form-factor. The latter does not represent an observable quantity. Expressing the function $F(q)$ as

$$
F\left(q^{2}\right)=\left(\alpha_{1}-\alpha_{2}+\alpha_{3}\right) M_{\pi}^{4}+\left(q^{2}+M_{\pi}^{2}\right)\left(\alpha_{2} M_{\pi}^{2}+\alpha_{3}\left(q^{2}-M_{\pi}^{2}\right)\right)
$$

the $\mathrm{N}^{3} \mathrm{LO}$ contribution reduces to a renormalization of the 1PE potential (2.9) and contact interactions. Notice that one also encounters an additional correction to the GoldbergerTreiman discrepancy. In addition, one has to take into account relativistic $\left(1 / \mathrm{m}^{2}\right)$ corrections to the static 1PE potential, which depend on a particular choice of the unitary transformation in the NN system and the form of the scattering (or bound state) equation, see Ref. [25] for details. The final expression for the 1PE potential adopted in the present work takes the form

$$
V_{1 \pi}(q)=-\left(\frac{g_{A}}{2 F_{\pi}}\right)^{2}\left(1-\frac{p^{2}+p^{\prime 2}}{2 m^{2}}\right) \vec{\tau}_{1} \cdot \vec{\tau}_{2} \frac{\vec{\sigma}_{1} \cdot \vec{q} \vec{\sigma}_{2} \cdot \vec{q}}{q^{2}+M_{\pi}^{2}},
$$

where, as in our previous work [21], we take the larger value $g_{A}=1.29$ instead of $g_{A}=$ 1.26 in order to account for the Goldberger-Treiman discrepancy. This corresponds to the pion-nucleon coupling constant $g_{\pi N}=13.1$.

We now turn to the $2 \mathrm{PE}$ contributions. The $2 \mathrm{PE}$ potential $V_{2 \pi}^{(2)}+V_{2 \pi}^{(3)}$ is discussed in [13-16] and in [12] using an energy-dependent formalism. While dimensional regularization or equivalent schemes have been used in $[13,14,16]$ to calculate matrix elements of 
the potential, a finite momentum cut-off approach has been applied in [12]. The $\mathrm{N}^{3} \mathrm{LO}$ corrections have been recently obtained by Kaiser using dimensional regularization [18]. In the following, we will adopt the SFR method to obtain the nonpolynomial contributions to the 2PE potential with the short-range components being explicitly excluded, see [17] for more details. The expressions for the 2PE potential in the SFR scheme up to NNLO have already been given in [21]. To keep the presentation self-contained, we give below the corresponding (nonpolynomial) terms at NLO

$$
\begin{aligned}
W_{C}^{(2)}(q)= & -\frac{1}{384 \pi^{2} F_{\pi}^{4}} L^{\tilde{\Lambda}}(q) \\
& \times\left\{4 M_{\pi}^{2}\left(5 g_{A}^{4}-4 g_{A}^{2}-1\right)+q^{2}\left(23 g_{A}^{4}-10 g_{A}^{2}-1\right)+\frac{48 g_{A}^{4} M_{\pi}^{4}}{4 M_{\pi}^{2}+q^{2}}\right\} \\
V_{T}^{(2)}(q)= & -\frac{1}{q^{2}} V_{S}^{(2)}(q)=-\frac{3 g_{A}^{4}}{64 \pi^{2} F_{\pi}^{4}} L^{\tilde{\Lambda}}(q)
\end{aligned}
$$

and at NNLO

$$
\begin{aligned}
& V_{C}^{(3)}(q)=-\frac{3 g_{A}^{2}}{16 \pi F_{\pi}^{4}}\left\{2 M_{\pi}^{2}\left(2 c_{1}-c_{3}\right)-c_{3} q^{2}\right\}\left(2 M_{\pi}^{2}+q^{2}\right) A^{\tilde{\Lambda}}(q), \\
& W_{T}^{(3)}(q)=-\frac{1}{q^{2}} W_{S}^{(3)}(q)=-\frac{g_{A}^{2}}{32 \pi F_{\pi}^{4}} c_{4}\left(4 M_{\pi}^{2}+q^{2}\right) A^{\tilde{\Lambda}}(q),
\end{aligned}
$$

where the NLO and NNLO loop functions $L^{\tilde{\Lambda}}(q)$ and $A^{\tilde{\Lambda}}(q)$ are given by

$$
\begin{aligned}
& L^{\tilde{\Lambda}}(q)=\theta\left(\tilde{\Lambda}-2 M_{\pi}\right) \frac{\omega}{2 q} \ln \frac{\tilde{\Lambda}^{2} \omega^{2}+q^{2} s^{2}+2 \tilde{\Lambda} q \omega s}{4 M_{\pi}^{2}\left(\tilde{\Lambda}^{2}+q^{2}\right)}, \\
& \omega=\sqrt{q^{2}+4 M_{\pi}^{2}}, \quad s=\sqrt{\tilde{\Lambda}^{2}-4 M_{\pi}^{2}}
\end{aligned}
$$

and

$$
A^{\tilde{\Lambda}}(q)=\theta\left(\tilde{\Lambda}-2 M_{\pi}\right) \frac{1}{2 q} \arctan \frac{q\left(\tilde{\Lambda}-2 M_{\pi}\right)}{q^{2}+2 \tilde{\Lambda} M_{\pi}} .
$$

The $\mathrm{N}^{3} \mathrm{LO}$ corrections to the $2 \mathrm{PE}$ potential $V_{2 \pi}^{(4)}$ have been recently calculated by Kaiser [18]. They arise from the one-loop "bubble" diagrams with both dimension two $\pi \pi N N$ vertices of the $c_{1, \ldots, 4}$-type and from the diagrams which contain the third order pionnucleon amplitude and lead to one-loop and two-loop graphs. We begin with the first group of corrections, for which one finds:

$$
\begin{aligned}
& V_{C}^{(4)}(q)=\frac{3}{16 \pi^{2} F_{\pi}^{4}} L^{\tilde{\Lambda}}(q)\left\{\left[\frac{c_{2}}{6} \omega^{2}+c_{3}\left(2 M_{\pi}^{2}+q^{2}\right)-4 c_{1} M_{\pi}^{2}\right]^{2}+\frac{c_{2}^{2}}{45} \omega^{4}\right\}, \\
& W_{T}^{(4)}(q)=-\frac{1}{q^{2}} W_{S}^{(4)}(q)=\frac{c_{4}^{2}}{96 \pi^{2} F_{\pi}^{4}} \omega^{2} L^{\tilde{\Lambda}}(q) .
\end{aligned}
$$


No closed expressions can be given for some of the corrections from the second group. ${ }^{3}$ It appears to be convenient to give the contributions to the potential using the (subtracted) spectral function representation:

$$
\begin{aligned}
& V_{C, S}(q)=-\frac{2 q^{6}}{\pi} \int_{2 M_{\pi}}^{\infty} d \mu \frac{\rho_{C, S}(\mu)}{\mu^{5}\left(\mu^{2}+q^{2}\right)}, \\
& V_{T}(q)=\frac{2 q^{4}}{\pi} \int_{2 M_{\pi}}^{\infty} d \mu \frac{\rho_{T}(\mu)}{\mu^{3}\left(\mu^{2}+q^{2}\right)} \\
& W_{C, S}(q)=-\frac{2 q^{6}}{\pi} \int_{2 M_{\pi}}^{\infty} d \mu \frac{\eta_{C, S}(\mu)}{\mu^{5}\left(\mu^{2}+q^{2}\right)}, \\
& W_{T}(q)=\frac{2 q^{4}}{\pi} \int_{2 M_{\pi}}^{\infty} d \mu \frac{\eta_{T}(\mu)}{\mu^{3}\left(\mu^{2}+q^{2}\right)} .
\end{aligned}
$$

For the spectral functions $\rho_{i}(\mu)\left(\eta_{i}(\mu)\right)$ one finds [18]

$$
\begin{aligned}
& \rho_{C}^{(4)}(\mu)=-\frac{3 g_{A}^{4}\left(\mu^{2}-2 M_{\pi}^{2}\right)}{\pi \mu\left(4 F_{\pi}\right)^{6}} \theta(\tilde{\Lambda}-\mu) \\
& \times\left\{\left(M_{\pi}^{2}-2 \mu^{2}\right)\left[2 M_{\pi}+\frac{2 M_{\pi}^{2}-\mu^{2}}{2 \mu} \ln \frac{\mu+2 M_{\pi}}{\mu-2 M_{\pi}}\right]\right. \\
& \left.+4 g_{A}^{2} M_{\pi}\left(2 M_{\pi}^{2}-\mu^{2}\right)\right\}, \\
& \eta_{S}^{(4)}(\mu)=\mu^{2} \eta_{T}^{(4)}(\mu) \\
& =-\frac{g_{A}^{4}\left(\mu^{2}-4 M_{\pi}^{2}\right)}{\pi\left(4 F_{\pi}\right)^{6}} \theta(\tilde{\Lambda}-\mu) \\
& \times\left\{\left(M_{\pi}^{2}-\frac{\mu^{2}}{4}\right) \ln \frac{\mu+2 M_{\pi}}{\mu-2 M_{\pi}}+\left(1+2 g_{A}^{2}\right) \mu M_{\pi}\right\}, \\
& \rho_{S}^{(4)}(\mu)=\mu^{2} \rho_{T}^{(4)}(\mu) \\
& =-\theta(\tilde{\Lambda}-\mu)\left\{\frac{g_{A}^{2} r^{3} \mu}{8 F_{\pi}^{4} \pi}\left(\bar{d}_{14}-\bar{d}_{15}\right)-\frac{2 g_{A}^{6} \mu r^{3}}{\left(8 \pi F_{\pi}^{2}\right)^{3}}\left[\frac{1}{9}-J_{1}+J_{2}\right]\right\}, \\
& \eta_{C}^{(4)}(\mu)=\theta(\tilde{\Lambda}-\mu)\left\{\frac{r t^{2}}{24 F_{\pi}^{4} \mu \pi}\left[2\left(g_{A}^{2}-1\right) r^{2}-3 g_{A}^{2} t^{2}\right]\left(\bar{d}_{1}+\bar{d}_{2}\right)\right. \\
& +\frac{r^{3}}{60 F_{\pi}^{4} \mu \pi}\left[6\left(g_{A}^{2}-1\right) r^{2}-5 g_{A}^{2} t^{2}\right] \bar{d}_{3}
\end{aligned}
$$

\footnotetext{
3 Entem and Machleidt were able to calculate most of the integrals in Eqs. (2.20) analytically (for $\tilde{\Lambda}=\infty$ ) and to express the corresponding contributions to the potential in terms of the loop functions $L^{\infty}(q)$ and $A^{\infty}(q)$ [26].
} 


$$
\begin{aligned}
& -\frac{r M_{\pi}^{2}}{6 F_{\pi}^{4} \mu \pi}\left[2\left(g_{A}^{2}-1\right) r^{2}-3 g_{A}^{2} t^{2}\right] \bar{d}_{5} \\
& -\frac{1}{92160 F_{\pi}^{6} \mu^{2} \pi^{3}}\left[-320\left(1+2 g_{A}^{2}\right)^{2} M_{\pi}^{6}+240\left(1+6 g_{A}^{2}+8 g_{A}^{4}\right) M_{\pi}^{4} \mu^{2}\right. \\
& -60 g_{A}^{2}\left(8+15 g_{A}^{2}\right) M_{\pi}^{2} \mu^{4} \\
& \left.+\left(-4+29 g_{A}^{2}+122 g_{A}^{4}+3 g_{A}^{6}\right) \mu^{6}\right] \ln \frac{2 r+\mu}{2 M_{\pi}} \\
& -\frac{r}{2700 \mu\left(8 \pi F_{\pi}^{2}\right)^{3}}\left[-16\left(171+2 g_{A}^{2}\left(1+g_{A}^{2}\right)\left(327+49 g_{A}^{2}\right)\right) M_{\pi}^{4}\right. \\
& +4\left(-73+1748 g_{A}^{2}+2549 g_{A}^{4}+726 g_{A}^{6}\right) M_{\pi}^{2} \mu^{2} \\
& \left.-\left(-64+389 g_{A}^{2}+1782 g_{A}^{4}+1093 g_{A}^{6}\right) \mu^{4}\right] \\
& \left.+\frac{2 r}{3 \mu\left(8 \pi F_{\pi}^{2}\right)^{3}}\left[g_{A}^{6} t^{4} J_{1}-2 g_{A}^{4}\left(2 g_{A}^{2}-1\right) r^{2} t^{2} J_{2}\right]\right\}
\end{aligned}
$$

where we have introduced the abbreviations

$$
r=\frac{1}{2} \sqrt{\mu^{2}-4 M_{\pi}^{2}}, \quad t=\sqrt{\mu^{2}-2 M_{\pi}^{2}},
$$

and

$$
\begin{aligned}
& J_{1}=\int_{0}^{1} d x\left\{\frac{M_{\pi}^{2}}{r^{2} x^{2}}-\left(1+\frac{M_{\pi}^{2}}{r^{2} x^{2}}\right)^{3 / 2} \ln \frac{r x+\sqrt{M_{\pi}^{2}+r^{2} x^{2}}}{M_{\pi}}\right\}, \\
& J_{2}=\int_{0}^{1} d x x^{2}\left\{\frac{M_{\pi}^{2}}{r^{2} x^{2}}-\left(1+\frac{M_{\pi}^{2}}{r^{2} x^{2}}\right)^{3 / 2} \ln \frac{r x+\sqrt{M_{\pi}^{2}+r^{2} x^{2}}}{M_{\pi}}\right\} .
\end{aligned}
$$

We use the scale-independent LECs $\bar{d}_{1}, \bar{d}_{2}, \bar{d}_{3}, \bar{d}_{5}, \bar{d}_{14}$ and $\bar{d}_{15}$ defined in [23]. In addition, one has to take into account the leading relativistic $(1 / \mathrm{m})$-corrections to the $2 \mathrm{PE}$ potential:

$$
\begin{aligned}
V_{C}^{(4)}(q)= & \frac{3 g_{A}^{4}}{512 \pi m F_{\pi}^{4}}\left\{\frac{2 M_{\pi}^{5}}{\omega^{2}}-3\left(4 M_{\pi}^{4}-q^{4}\right) A^{\tilde{\Lambda}}(q)\right\} \\
W_{C}^{(4)}(q)= & \frac{g_{A}^{2}}{128 \pi m F_{\pi}^{4}}\left\{\frac{3 g_{A}^{2} M_{\pi}^{5}}{\omega^{2}}-\left[4 M_{\pi}^{2}+2 q^{2}-g_{A}^{2}\left(7 M_{\pi}^{2}+\frac{9}{2} q^{2}\right)\right]\right. \\
& \left.\times\left(2 M_{\pi}^{2}+q^{2}\right) A^{\tilde{\Lambda}}(q)\right\} \\
V_{T}^{(4)}(q)= & -\frac{1}{q^{2}} V_{S}^{(4)}(q)=\frac{9 g_{A}^{2}}{512 \pi m F_{\pi}^{4}}\left(4 M_{\pi}^{2}+\frac{3}{2} q^{2}\right) A^{\tilde{\Lambda}}(q), \\
W_{T}^{(4)}(q)= & -\frac{1}{q^{2}} W_{S}^{(4)}(q) \\
= & -\frac{g_{A}^{2}}{256 \pi m F_{\pi}^{4}}\left[8 M_{\pi}^{2}+2 q^{2}-g_{A}^{2}\left(8 M_{\pi}^{2}+\frac{5}{2} q^{2}\right)\right] A^{\tilde{\Lambda}}(q),
\end{aligned}
$$




$$
\begin{aligned}
& V_{L S}^{(4)}(q)=-\frac{3 g_{A}^{4}}{64 \pi m F_{\pi}^{4}}\left(2 M_{\pi}^{2}+q^{2}\right) A^{\tilde{\Lambda}}(q), \\
& W_{L S}^{(4)}(q)=-\frac{g_{A}^{2}\left(1-g_{A}^{2}\right)}{64 \pi m F_{\pi}^{4}}\left(4 M_{\pi}^{2}+q^{2}\right) A^{\tilde{\Lambda}}(q) .
\end{aligned}
$$

Notice that these relativistic corrections differ from the ones given in Ref. [14]. In fact, the specific form of the terms in Eq. (2.23) depends on the form of the Schrödinger (or Lippmann-Schwinger) equation, see [25] for more details. The relativistic corrections given in Eq. (2.23) are consistent with the relativistic Schrödinger equation (A.4) and with the $\left(1 / \mathrm{m}^{2}\right)$-corrections to the 1PE potential in Eq. (2.13).

Three-pion exchange starts to contribute at $\mathrm{N}^{3} \mathrm{LO}$. The corresponding expressions for the spectral functions and the potential (obtained using dimensional regularization) have been given by Kaiser in [19,20,27]. It has been pointed out in these references that the $3 \mathrm{PE}$ potential is much weaker than the $\mathrm{N}^{3} \mathrm{LO} 2 \mathrm{PE}$ contributions at physically interesting distances $r>1 \mathrm{fm}$. Having the explicit expressions for the 3PE spectral functions, it is easy to calculate the potential in the SFR scheme. It is obvious even without performing the explicit calculations that the finite-range part of the 3PE potential in the SFR scheme is strongly suppressed at intermediate and short distances compared to the result obtained using DR. This is because the short range components which dominate the 3PE spectrum are explicitly excluded in this approach. To illustrate that let us consider the isoscalar spinspin contribution proportional to $g_{A}^{4}$, which has been found in [20] to provide the strongest $3 \mathrm{PE}$ potential for $0.6 \mathrm{fm}<r<1.4 \mathrm{fm}$. The corresponding DR spectral functions $\rho_{S, 3 \pi}^{\mathrm{DR}}(\mu)$ and $\rho_{T, 3 \pi}^{\mathrm{DR}}(\mu)$ are given by [20]

$$
\begin{aligned}
\rho_{S, 3 \pi}^{\mathrm{DR}}(\mu)= & -\frac{g_{A}^{4}\left(\mu-3 M_{\pi}\right)^{2}}{35 \pi\left(32 F_{\pi}^{3}\right)^{2}}\left[2 M_{\pi}^{2}-12 \mu M_{\pi}-2 \mu^{2}+15 \frac{M_{\pi}^{3}}{\mu}+2 \frac{M_{\pi}^{4}}{\mu^{2}}+3 \frac{M_{\pi}^{5}}{\mu^{3}}\right], \\
\rho_{T, 3 \pi}^{\mathrm{DR}}(\mu)= & -\frac{g_{A}^{4}\left(\mu-3 M_{\pi}\right)}{35 \pi\left(32 \mu F_{\pi}^{3}\right)^{2}} \\
& \times\left[\mu^{3}+3 \mu^{2} M_{\pi}+2 \mu M_{\pi}^{2}+6 M_{\pi}^{3}+18 \frac{M_{\pi}^{4}}{\mu}-9 \frac{M_{\pi}^{5}}{\mu^{2}}-27 \frac{M_{\pi}^{6}}{\mu^{3}}\right] .
\end{aligned}
$$

The finite-range part of the spin-spin potential $V_{S}(r)$ can be obtained from the corresponding spectral functions via

$$
V_{S}(r)=-\frac{1}{6 \pi^{2} r} \int_{3 M_{\pi}}^{\infty} d \mu \mu e^{-\mu r}\left(\mu^{2} \rho_{T}(\mu)-3 \rho_{S}(\mu)\right) .
$$

Using the spectral functions in Eq. (2.24) one then gets for $V_{S, 3 \pi}^{\mathrm{DR}}(r)$ :

$$
V_{S, 3 \pi}^{\mathrm{DR}}(r)=\frac{g_{A}^{4}}{2\left(8 \pi F_{\pi}^{2}\right)^{3}} \frac{e^{-3 M_{\pi} r}}{r^{7}}\left(1+M_{\pi} r\right)^{2}\left(2+M_{\pi} r\right)^{2} .
$$

Introducing the cut-off $\tilde{\Lambda}$ in the spectral functions via $\rho_{S, 3 \pi}^{\mathrm{SFR}}(\mu)=\rho_{S, 3 \pi}^{\mathrm{DR}}(\mu) \theta(\tilde{\Lambda}-\mu)$ and $\rho_{T, 3 \pi}^{\mathrm{SFR}}(\mu)=\rho_{T, 3 \pi}^{\mathrm{DR}}(\mu) \theta(\tilde{\Lambda}-\mu)$ we obtain for the SFR potential $V_{S, 3 \pi}^{\mathrm{SFR}}(r)$ : 


$$
\begin{aligned}
V_{S, 3 \pi}^{\mathrm{SFR}}(r)= & V_{S, 3 \pi}^{\mathrm{DR}}(r)-\frac{g_{A}^{4}}{30\left(32 \pi F_{\pi}^{2}\right)^{3}} \frac{e^{-3 y}}{r^{7}}\left[120+120 y+60 y^{2}+20 y^{3}+5 y^{4}+y^{5}\right. \\
& -25 M_{\pi}^{2} r^{2}\left(6+6 y+3 y^{2}+y^{3}\right)+45 M_{\pi}^{3} r^{3}\left(2+2 y+y^{2}\right) \\
& \left.+30 M_{\pi}^{4} r^{4}(1+y)-63 M_{\pi}^{5} r^{5}\right]
\end{aligned}
$$

where we have introduced the abbreviation $y=\tilde{\Lambda} r$. In Fig. 1 we plot $V_{S, 3 \pi}^{\mathrm{DR}}(r)$ and $V_{S, 3 \pi}^{\mathrm{SFR}}(r)$ for $r$ from 0.5 to $2 \mathrm{fm}$. The potential calculated using the spectral function regularization is much smaller in magnitude compared to the one obtained using dimensional regularization. Clearly, such a suppression does not take place at very large $r$, where $V_{S, 3 \pi}^{\mathrm{SFR}}(r)$ approaches $V_{S, 3 \pi}^{\mathrm{DR}}(r)$. At such distances, however, the 3PE potential becomes negligibly small compared to the 1PE and 2PE contributions simply due to its shorter range. As a consequence, the 3PE potential can be neglected everywhere except the region of very small $r$, where it anyhow becomes unreliable. This is further exemplified in Fig. 2, where we show the ratio of the $\mathrm{N}^{3} \mathrm{LO}$ isoscalar spin-spin contributions of 3PE and 2PE using both regularization schemes for a wide range of $r$. It turns out that the 3PE contribution reaches for $r>0.5 \mathrm{fm}$ at most $2 \%-8 \%$ of the corresponding $\mathrm{N}^{3} \mathrm{LO} 2 \mathrm{PE}$ contribution depending on the choice of the spectral function cut-off. We therefore neglect all 3PE contributions in the present analysis.

Although we have regularized the $2 \mathrm{PE}$ contributions by cutting off the large-mass components in the spectrum (or, equivalently, by explicitly shifting the corresponding shortdistance components to contact terms), the resulting potential still behaves incorrectly at large momenta (or equivalently at short distances). The effective potential is valid for small values of the momentum transfer $q$ and becomes meaningless for momenta $q \gtrsim \Lambda_{\chi}$. Moreover, since the potential $V$ grows with increasing momenta $q$, the scattering equation is ultraviolet divergent and needs to be regularized. Following the standard procedure, see,

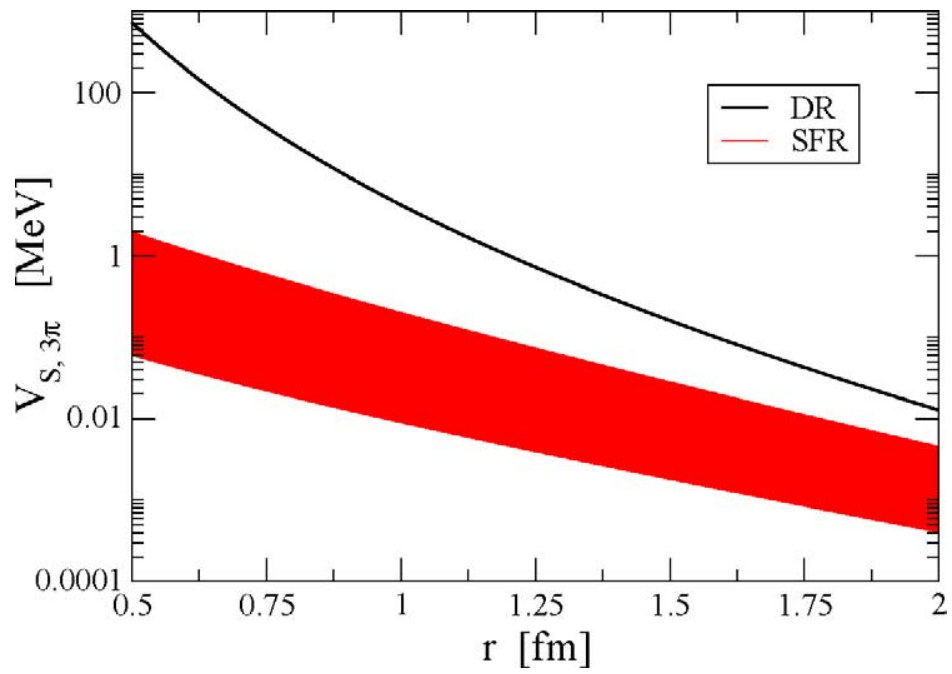

Fig. 1. Isoscalar spin-spin 3PE potential using dimensional (DR) and spectral function regularization (SFR). The cut-off in the spectral function varies in the range $\tilde{\Lambda}=500-700 \mathrm{MeV}$. 


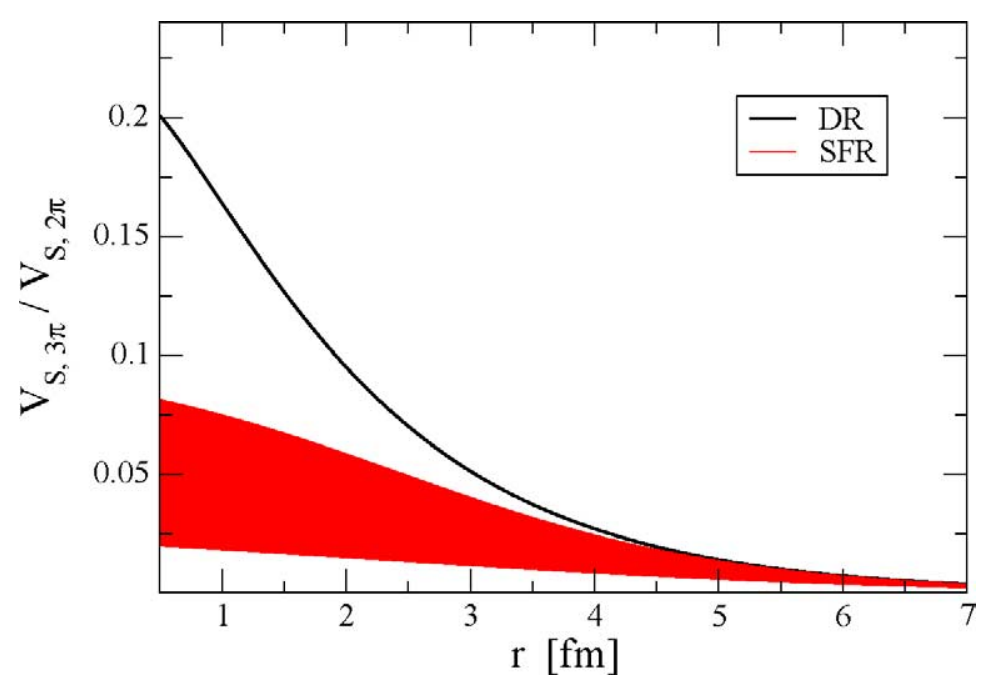

Fig. 2. The ratio of the isoscalar spin-spin $3 \mathrm{PE}$ and $2 \mathrm{PE} \mathrm{N}{ }^{3} \mathrm{LO}$ contributions using dimensional (DR) and spectral function regularization (SFR). The cut-off in the spectral function varies in the range $\tilde{\Lambda}=500-700 \mathrm{MeV}$.

e.g., [16], we introduce an additional cut-off in the LS equation by multiplying the potential $V\left(\vec{p}, \vec{p}^{\prime}\right)$ with a regulator function $f^{\Lambda}$,

$$
V\left(\vec{p}, \vec{p}^{\prime}\right) \rightarrow f^{\Lambda}(p) V\left(\vec{p}, \vec{p}^{\prime}\right) f^{\Lambda}\left(p^{\prime}\right) .
$$

In what follows, we use the exponential regulator function

$$
f^{\Lambda}(p)=\exp \left[-p^{6} / \Lambda^{6}\right]
$$

We will specify the values of the cut-offs below.

It should be understood that our treatment of the effective potential is based on the heavy baryon formalism. As demonstrated in [28,29], heavy baryon expansion becomes formally invalid for certain two-pion exchange contributions at very large distances. This problem with the heavy baryon formalism has been first observed in the single-nucleon sector and can be dealt with using, e.g., the Lorentz invariant scheme proposed by Becher and Leutwyler [30]. It is clear, however, that the $N N$ interaction due to two-pion exchange becomes very weak at large distances, so that the problem with the formal inconsistency of the heavy baryon approach is expected to have little relevance for practical applications.

Last but not least, we would like to comment on some key features of the SFR scheme adopted in the present work. First of all, it is crucial to understand that this approach does not affect the "chiral features" of the $N N$ potential. We remind the reader that the resulting effective $N N$ potential consists of the long- and short-range pieces. Spontaneously broken approximate chiral symmetry of QCD leads to highly nontrivial constraints for the longrange part of the potential, which is given by the terms nonpolynomial in momenta. The short-range part of the potential given by a series of the most general contact interactions with increasing power of momenta is not affected by chiral symmetry with the exception of the quark-mass dependence of the corresponding LECs, which is not relevant for the present analysis. In other words, only the long-distance asymptotics of the potential is 
constrained by chiral symmetry. The SFR scheme does, per construction, not affect the long-distance asymptotics of the potential and leads to the same result as obtained using DR, see [17] for more details. The only difference to the DR result is given by a series of the short-range interactions. It is, therefore, obvious, that the SFR method does not affect the constraints of the chiral symmetry implemented in the $N N$ potential. Further, we point out that the equivalence of the SFR and the finite cut-off regularization has only been established at a one-loop level and does not hold true for both loop integrals of the $\mathrm{N}^{3} \mathrm{LO}$ 2PE contribution. The prominent feature of the applied regularization scheme is given by the fact, that it only affects the two-nucleon interaction. One can, therefore, directly adopt the values for various LECs resulting from the single-nucleon sector analyses, where dimensional regularization has been used. On the contrary, if a finite momentum cut-off regularization would be applied to both loop integrals entering the $\mathrm{N}^{3} \mathrm{LO} 2 \mathrm{PE}$ contribution, one would need to re-extract the values of the corresponding LECs from pion-nucleon scattering and the process $\pi N \rightarrow \pi \pi N$ using the same regularization scheme.

\subsection{Isospin-breaking effects}

Isospin-breaking nuclear forces have been extensively studied within effective field theory approaches, see, e.g., [7,31-35], as well as using more phenomenological methods, see, e.g., [36,37] for some recent references. In the Standard Model, isospin-violating effects have their origin in both strong (i.e., due to the different masses of the up and down quarks) and electromagnetic interactions (due to different charges of the up and down quarks). The electromagnetic effects can be separated into the ones due to soft and hard photons. While effects of hard photons are incorporated in effective field theory by inclusion of electromagnetic short distance operators in the effective Lagrangian, soft photons have to be taken into account explicitly.

In the present analysis we are rather limited in the treatment of isospin-violating interaction, which have to be included precisely in the way it is done by the Nijmegen group [38]. This is due to the fact that we are using the Nijmegen phase shifts instead of the real data as an input to fit the unknown LECs. Let us explain this point in more detail. With the only exception of the ${ }^{1} S_{0}$ partial wave, the $n p$ isovector phase shifts in the Nijmegen PWA are not obtained independently from $n p$ data, but rather extracted from the proton-proton $(p p)$ phase shifts using the assumption that the differences in the phase shifts result entirely due to isospin-breaking effects associated with $m_{p} \neq m_{n}$ and $M_{\pi^{ \pm}} \neq M_{\pi^{0}}$ in the 1PE potential as well as due to electromagnetic interactions. In order to be consistent with the Nijmegen phase shift analysis, we therefore have to neglect various isospin-breaking corrections and adopt the same isospin-breaking and electromagnetic interactions as in [38]. Nevertheless, we have decided to overview the dominant isospin-breaking contributions and to remind the reader on their relative size following mainly the lines of Ref. [7] but extending the consideration to higher orders. For a detailed review of charge-symmetry breaking in the nucleon-nucleon interaction the reader is referred to [35].

Consider first isospin breaking in the strong interaction. The QCD quark mass term can be expressed as

$$
\mathcal{L}_{\text {mass }}^{\mathrm{QCD}}=-\frac{1}{2} \bar{q}\left(m_{u}+m_{d}\right)\left(1-\epsilon \tau_{3}\right) q,
$$


where

$$
\epsilon \equiv \frac{m_{d}-m_{u}}{m_{d}+m_{u}} \sim \frac{1}{3} .
$$

The above numerical estimation is based on the light quark mass values utilizing a modified $\overline{\mathrm{MS}}$ subtraction scheme at a renormalization scale of $1 \mathrm{GeV}$. The isoscalar term in Eq. (2.30) breaks chiral but preserves isospin symmetry. It leads to the nonvanishing pion mass, $M_{\pi}^{2}=\left(m_{u}+m_{d}\right) B \neq 0$, where $B$ is a low-energy constant that describes the strength of the bilinear light quark condensates. All chiral symmetry breaking interactions in the effective Lagrangian are proportional to positive powers of $M_{\pi}^{2}$. The isovector term $\left(\propto \tau_{3}\right)$ in Eq. (2.30) breaks isospin symmetry and generates a series of isospin-breaking effective interactions $\propto\left(\epsilon M_{\pi}^{2}\right)^{n}$ with $n \geqslant 1$. It therefore appears to be natural to count strong isospin violation in terms of $\epsilon M_{\pi}^{2}$. However, we note already here that isospin-breaking effects are in general much smaller than indicated by the numerical value of $\epsilon$, because the relevant scale for the isospin-conserving contributions is $\Lambda_{\chi}$ rather than $m_{u}+m_{d}$.

Electromagnetic terms in the effective Lagrangian can be generated using the method of external sources, see, e.g., [39-41] for more details. All such terms are proportional to the nucleon charge matrix $Q_{\mathrm{ch}}=e\left(1+\tau_{3}\right) / 2$, where $e$ denotes the electric charge. ${ }^{4}$ More precisely, the vertices which contain (do not contain) the photon fields are proportional to $Q_{\mathrm{ch}}^{n}\left(Q_{\mathrm{ch}}^{2 n}\right)$, where $n=1,2, \ldots$ Since we are interested here in nucleon-nucleon scattering in the absence of external fields, so that no photon can leave a Feynman diagram, it is convenient to introduce the small parameter $e^{2} \sim 1 / 10$ for isospin-violating effects caused by the electromagnetic interactions.

Due to its perturbative nature induced by the small parameters $\epsilon M_{\pi}^{2}$ and $e^{2}$, we treat the strong and electromagnetic isospin violation in addition to the power counting of the isospin symmetric potential mentioned in Section 2.1. Although not necessary, in practical applications it often appears to be more convenient to have a single expansion parameter. Thus, one has to relate the quantities $\epsilon, e$ to the generic low-momentum scale $Q$ related to external three-momenta of nucleons and the pion mass $\left(p \sim p^{\prime} \sim M_{\pi} \sim Q\right)$ and introduced before. Here and below, we will make use of the following simple counting rules: ${ }^{5}$

$$
\epsilon \sim e \sim \frac{Q}{\Lambda_{\chi}} .
$$

The counting of the electric charge is consistent with the one commonly used in the pion and pion-nucleon sectors, see, e.g., [39,42-44] (it differs, however, from what is commonly used in the description of extremely nonrelativistic hadronic bound state, see, e.g., [45]). In addition to the above mentioned counting rules, we need to deal with the extra $1 /(4 \pi)^{2}$ factors, which typically arise when calculating loop integrals. For pion loops, such factors

\footnotetext{
4 Or equivalently, one can use the quark charge matrix $e\left(1 / 3+\tau_{3}\right) / 2$.

5 This suggests a slightly different counting of the strong isospin-breaking effects as compared to [7]. Most of the conclusions of [7] remain, however, unchanged. The important difference is that the leading isospin-violating short-range interaction is now proportional to the quark mass difference, while electromagnetic contact terms are shifted to higher orders.
} 
are naturally incorporated in the chiral power counting through the relation $\Lambda_{\chi} \sim 4 \pi F_{\pi}$. For photon loops we will further assume, that

$$
\frac{e^{2}}{(4 \pi)^{2}} \sim \frac{Q^{4}}{\Lambda_{\chi}^{4}},
$$

which simply means that the factors $1 /(4 \pi)^{2}$ provide two additional powers of the small parameter. In the following, we will denote the order of various isospin-violating interactions by " $\mathrm{\phi}$ ", "NL $\varnothing ",, \ldots$ in order to distinguish the above mentioned phenomenological extension of the counting rules from the usual chiral power counting in the isospinconserved case. Certainly, one has always the option to discard this generalization of the chiral counting rules and to perform separate expansions in $\epsilon, e$ and $Q / \Lambda_{\chi}$. Notice further that Eqs. (2.32) and (2.33) suggest a different counting of the strong isospin-breaking effects compared to Ref. [7]. In that work strong and electromagnetic effects have been classified using a separate expansion without introducing a unified expansion scheme. The $\mathrm{L} \phi, \mathrm{NL} \phi, \ldots$ contributions in the present work should therefore not be confused with the corresponding terms in [7]. Last but not least, the above counting scheme is similar to the one adopted, e.g., in [31,33], where effects $\sim \alpha / \pi$ were also considered as being one order suppressed compared to the ones $\sim \epsilon M_{\pi}^{2} / \Lambda_{\chi}^{2}$.

Let us now apply the power counting rules to estimate isospin-violating corrections to hadronic masses, see also [35] for a similar estimation. We begin with the pion mass. It is well known that the pion mass does not receive contributions linear in the quark mass difference and the strong contribution to the pion mass starts at the second order in $m_{d}-m_{u}$. Consequently, the leading strong term can be estimated as $\left(\Delta M_{\pi}^{2}\right)_{\operatorname{str}} \equiv$ $\left(M_{\pi^{ \pm}}^{2}-M_{\pi^{0}}^{2}\right)_{\text {str }} \propto\left(\epsilon M_{\pi}^{2}\right)^{2} \Lambda_{\chi}^{-2}$, which is of the order $v=6$ to be compared to $v=2$ for the isospin-symmetric term $\propto M_{\pi}^{2}$. One thus expects the strong contribution to the pion mass difference $\left(\Delta M_{\pi}\right)_{\text {str }} \equiv\left(M_{\pi^{ \pm}}-M_{\pi^{0}}\right)_{\text {str }}$ to have the size $\sim 0.1-0.3 \mathrm{MeV}$ depending on whether one substitutes in the numerical estimation $M_{\rho}$ or $4 \pi F_{\pi}$ for $\Lambda_{\chi}$. Clearly, we cannot predict whether the shift is positive or negative. The leading electromagnetic contribution to $\Delta M_{\pi}^{2}$ is of the order $\left(\Delta M_{\pi}^{2}\right)_{\mathrm{em}} \sim e^{2}(4 \pi)^{-2} \Lambda_{\chi}^{2}$, which is a $(v=4)$-effect according to our counting rules. Numerically, one estimates the size of the pion mass difference to be $\left(\Delta M_{\pi}\right)_{\mathrm{em}} \sim 1-3 \mathrm{MeV}$. We see that both power counting arguments and numerical estimations suggest that the pion mass difference is mainly of electromagnetic origin. Furthermore, the estimated size of the electromagnetic shift agrees well with the observed value $M_{\pi^{ \pm}}-M_{\pi^{0}}=4.6 \mathrm{MeV}$. All these statements can also be backed by hard calculations, for a classical review see [46]. For the nucleon mass difference, the strong contribution is linear in the quark mass differences and can be estimated as: $(\Delta m)_{\text {str }} \equiv\left(m_{n}-m_{p}\right)_{\text {str }} \sim\left(\epsilon M_{\pi}^{2}\right) \Lambda_{\chi}^{-1} \sim 6-9 \mathrm{MeV}{ }^{6}$ According to the counting rules, this is the $(v=3)$-effect. Electromagnetic shift appears at $v=4$ and is expected to be of the or$\operatorname{der}(\Delta m)_{\mathrm{em}} \sim e^{2}(4 \pi)^{-2} \Lambda_{\chi} \sim 0.5-0.7 \mathrm{MeV}$. In reality, the effects are of opposite sign and the difference between them is less pronounced. One observes $\left(m_{n}-m_{p}\right)_{\text {str }} \simeq 2.1 \mathrm{MeV}$

\footnotetext{
6 This too large value reflects our earlier statement about the use of the parameter $\epsilon$ to estimate isospin-breaking corrections.
} 
and $\left(m_{n}-m_{p}\right)_{\mathrm{em}} \simeq-0.8 \mathrm{MeV}$, leading to the physical value of $m_{n}-m_{p}=1.3 \mathrm{MeV}$ (see again [46] for more details).

We are now in the position to discuss various isospin-breaking contributions to the two-nucleon force. As explained in [7], the leading-order (i.e., L $\phi$ ) isospin-breaking interactions are due to the pion mass difference in the 1PE potential and the static Coulomb interaction. The latter is clearly of the order ${ }^{7} \sim e^{2} Q^{-2} F_{\pi}^{2}$, while the former is

$$
\sim \frac{\Delta M_{\pi}^{2}}{M_{\pi}^{2}} \sim\left(\frac{e^{2}}{(4 \pi)^{2}} \Lambda_{\chi}^{2}\right) \frac{1}{M_{\pi}^{2}}=\mathcal{O}\left[\frac{Q^{2}}{\Lambda_{\chi}^{2}}\right],
$$

where we used of the counting rules (2.32) and (2.33) together with $M_{\pi} \sim Q$. Thus, the $L \varnothing$ isospin-breaking force is of the order $v=2$. Consider now NL $\varnothing$ corrections to this result, which appear at $v=3$. The pion-nucleon coupling constant receives strong isospin-violating contributions of the order $\epsilon M_{\pi}^{2} / \Lambda_{\chi}^{2}$. The corresponding LECs in the pion-nucleon Lagrangian are denoted by $d_{17}, d_{18}$ and $d_{19}$ in the notation of Ref. [23]. This charge dependence of the pion-nucleon coupling constant leads to isospin-violating $1 \mathrm{PE}$ of the order $v=3$. In addition, one has to take into account strong isospin-breaking contact interaction of the kind

$$
\epsilon M_{\pi}^{2}\left(N^{\dagger} \tau_{3} N\right)\left(N^{\dagger} N\right),
$$

which leads to charge symmetry breaking. We can check the accuracy of our estimation numerically using the values for the LECs found in [7]. According to Eq. (2.35), we expect the ratio of the isospin-breaking terms to isospin-conserving ones to be typically of the size: $\epsilon M_{\pi}^{2} / \Lambda_{\chi}^{2} \sim 0.5 \%-1.1 \%$, where the uncertainty results again from using two different estimations for $\Lambda_{\chi}$. Picking up the numbers from Table 2 in [7] we find for this ratio the values $0.8 \%, 0.8 \%$ and $3.1 \%$ for three different values of the (sharp) cut-off $\Lambda$ in the Lippmann-Schwinger equation: $\Lambda=300, \Lambda=400$ and $\Lambda=500 \mathrm{MeV}$. Thus, our numerical estimation is consistent with the results of [7]. The NNL $\varnothing$ corrections are of the order $v=4$ and arise from various sources. First, one has to take into account isospin-breaking in the 2PE potential due to electromagnetic corrections to the pion-nucleon coupling, see, e.g., [33]. The correction due to the pion mass difference in the leading $2 \mathrm{PE}$ potential can be estimated as

$$
\sim \frac{\Delta M_{\pi}^{2}}{M_{\pi}^{2}} \frac{Q^{2}}{\Lambda_{\chi}^{2}}=\mathcal{O}\left[\frac{Q^{4}}{\Lambda_{\chi}^{4}}\right] .
$$

Another isospin-violating two-pion exchange interaction at this order is generated by the triangle and football diagrams with one insertion of the isospin-breaking $\pi \pi N N$ vertex with the LEC $c_{5}$ (using again the notation of [23]). This vertex is proportional to $\epsilon M_{\pi}^{2}$ and is thus formally of the lower order than the electromagnetic $\pi \pi N N$ vertices $\propto e^{2} /(4 \pi)^{2}$. As we have seen on an example of the nucleon mass difference, in practice, both effects might be of a comparable size. For a recent work on this kind of isospin-breaking forces

\footnotetext{
7 The factor $F_{\pi}^{2}$ results from the common normalization of the isospin-symmetric part of the two-nucleon potential adopted in this work. This factor can be understood, e.g., from looking at the 1PE potential in Eq. (2.9).
} 
see [37]. The remaining contributions are given by the static $\pi \gamma$-exchange of the order $\sim e^{2} /(4 \pi)^{2}$ and by two independent contact interactions

$$
\frac{e^{2}}{(4 \pi)^{2}}\left(N^{\dagger} \tau_{3} N\right)\left(N^{\dagger} \tau_{3} N\right) \text { and } \frac{e^{2}}{(4 \pi)^{2}}\left(N^{\dagger} \tau_{3} N\right)\left(N^{\dagger} N\right)
$$

which lead to both charge independence and charge symmetry breaking. Notice that, in practice, the effect of the second interaction cannot be disentangled from the effect of term in Eq. (2.35).

Let us now estimate the size of isospin violation in the NN scattering due to the nucleon mass difference. We first note that the first relativistic corrections to the isospin-symmetric part of the two-nucleon force appear at $\mathrm{N}^{3} \mathrm{LO}(v=4)$ and are given by $\left(1 / \mathrm{m}^{2}\right)$-corrections to the $1 \mathrm{PE}$ and $(1 / \mathrm{m})$-corrections to the leading $2 \mathrm{PE}$ potential. Consequently, the size of the corresponding isospin-violating terms can be estimated as

$$
\frac{\Delta m}{m} \frac{Q^{4}}{\Lambda_{\chi}^{4}} \sim \frac{\epsilon M_{\pi}^{2}}{m \Lambda_{\chi}} \frac{Q^{4}}{\Lambda_{\chi}^{4}}=\mathcal{O}\left[\frac{Q^{8}}{\Lambda_{\chi}^{8}}\right] .
$$

Such terms therefore contribute only at the order $v=8$. In addition to the above mentioned corrections, one has to account for the fact that the neutron-proton mass difference leads to energy shifts of virtual states when calculating two-pion exchange diagrams. This can also easily been understood in the language of the heavy baryon formalism: factoring out the exponential factor $\exp \left(\operatorname{im}_{p} v \cdot x\right)$ from the proton and neutron fields, where $v$ and $x$ denote the proton velocity and position, the neutron propagator receives a shift in the denominator $\propto\left(m_{n}-m_{p}\right)$ after integrating out the small field components. It is then easy to see that the isospin-violating $2 \mathrm{PE}$ is suppressed against its isospin-conserving part by a factor:

$$
\sim \frac{\Delta m}{Q} \sim \frac{\epsilon M_{\pi}^{2}}{\Lambda_{\chi} Q}=\mathcal{O}\left[\frac{Q^{2}}{\Lambda_{\chi}^{2}}\right] .
$$

Therefore, neutron-proton mass difference in 2PE starts to contribute at $v=4$. This sort of charge symmetry breaking corrections has been studied recently in [35,36]. Notice further that, as pointed out in [35], certain loop integrals in the $2 \mathrm{PE}$ contributions give only one power of $(4 \pi)$ instead of expected two powers and are, therefore, enhanced. We will not take this enhancement into account in the present work. Apart from the above mentioned corrections to the nucleon-nucleon force, the neutron-proton mass difference has to be taken into account in kinematical relations as discussed in Appendix A, as well as in the expression for the kinetic energy of the nucleons. Let us consider this last effect. Its contribution to the scattering amplitude can be estimated by looking at the Lippmann-Schwinger equation

$$
T=V+V G_{0} T,
$$

where $G_{0}$ refers to the free propagator of two nucleons. Both terms on the right-hand side of the above equation are of the same order $v=0$. Taking into account the nucleon mass difference in $G_{0}$ leads therefore to a correction to the $T$-matrix of the order

$$
\sim \frac{\Delta m}{m} \sim \frac{\epsilon M_{\pi}^{2}}{m \Lambda_{\chi}}=\mathcal{O}\left[\frac{Q^{4}}{\Lambda_{\chi}^{4}}\right],
$$


and thus contribute at $v=4$. Notice that this estimation is valid for both relativistic and nonrelativistic expressions for the two-nucleon propagator $G_{0}$.

All other isospin-violating corrections are suppressed by further powers of the small parameter. We would like, however, to point out an important limitation of our estimation due to the fact that we do not explicitly account for the long-range nature of electromagnetic forces. Consider, for example, the leading one-pion and one-photon exchange forces. For simplicity, we will restrict ourselves to the ${ }^{1} S_{0}$ proton-proton channel, where the $1 \mathrm{PE}$ potential takes the form

$$
V_{1 \pi}^{(0)}(q)=\left(\frac{g_{A}}{2 F_{\pi}}\right)^{2} \frac{q^{2}}{q^{2}+M_{\pi}^{2}} .
$$

The static Coulomb interaction

$$
V_{\text {Coulomb }}(q)=\frac{e^{2}}{q^{2}},
$$

is suppressed compared to $V_{1 \pi}^{(0)}(q)$ by two powers of the small parameter $Q / \Lambda_{\chi}$ according to the power counting. Such an estimation works fairly well for momenta $q$ of the order $q \sim M_{\pi}$, for which we get $V_{1 \pi}^{(0)}\left(q=M_{\pi}\right) \sim 23 \mathrm{GeV}^{-2}$ and $V_{\text {Coulomb }}\left(q=M_{\pi}\right) \sim 5 \mathrm{GeV}^{-2}$. The power counting, however, breaks down for small momenta $q \ll M_{\pi}$ due to the long-range nature of the Coulomb interaction. For example, for $q=M_{\pi} / 4$ one gets: $V_{1 \pi}^{(0)}\left(q=M_{\pi} / 4\right) \sim 3 \mathrm{GeV}^{-2}$ while $V_{\text {Coulomb }}\left(q=M_{\pi} / 4\right) \sim 82 \mathrm{GeV}^{-2}$. Consequently, the Coulomb interaction provides the dominant contribution to the potential for small momenta and requires a nonperturbative treatment at low energy. A possible way out of the above mentioned inconsistency would be to develop separate and systematic power counting for momenta much smaller than the pion mass. This is, however, beyond the scope of the present work. Notice that a similar idea with two different power counting regimes has been applied recently to the nucleon Compton scattering in order to extend the region of applicability of the effective field theory in the $\Delta$-region [47]. In the present analysis, we will simply take into account higher-order corrections to the long-range electromagnetic interactions when determining the values of the LECs in order to correct for the low-momentum behavior of the $N N$ potential. The first long-range corrections beyond the ones considered above result from two-photon exchange, whose size can be estimated as

$$
\sim \frac{e^{2}}{Q^{2}} \frac{e^{2}}{(4 \pi)^{2}} F_{\pi}^{2}=\mathcal{O}\left[\frac{Q^{6}}{\Lambda_{\chi}^{6}}\right] .
$$

It thus formally appears at the order $v=6$. In addition, at the same order $v=6$ one has to take into account relativistic $(1 / m)$ corrections to the static one-photon exchange, which provide a contribution of the following size:

$$
\sim \frac{e^{2}}{Q^{2}} F_{\pi}^{2} \frac{Q^{2}}{m^{2}}=\frac{e^{2}}{m^{2}} F_{\pi}^{2}=\mathcal{O}\left[\frac{Q^{6}}{\Lambda_{\chi}^{6}}\right] .
$$

The relative sizes of various isospin-breaking contributions discussed above are summarized in Table 1. In what follows, we will give explicit expressions for the above mentioned interactions. 
Table 1

Dominant contributions to the isospin-symmetric and isospin-breaking parts of the two-nucleon force

\begin{tabular}{|c|c|c|}
\hline Isospin-symmetric & Isospin-breaking, finite-range & Isospin-breaking, long-range \\
\hline $\begin{array}{l}\mathrm{LO}(v=0) \text { : } \\
\text { static 1PE, contact terms without } \\
\text { derivatives }\end{array}$ & - & - \\
\hline $\begin{array}{l}\mathrm{NLO}(v=2) \text { : } \\
\text { leading } 2 \mathrm{PE} \text {, contact terms with } 2 \\
\text { derivatives }\end{array}$ & $\begin{array}{l}\mathrm{L} \phi(v=2): \\
M_{\pi^{ \pm}} \neq M_{\pi^{0}} \text { in } 1 \mathrm{PE}\end{array}$ & $\begin{array}{l}\mathrm{L} \phi(\nu=2) \text { : } \\
\text { static } 1 \gamma \text {-exchange }\end{array}$ \\
\hline $\begin{array}{l}\text { NNLO }(v=3) \text { : } \\
\text { subleading } 2 \mathrm{PE}\end{array}$ & $\begin{array}{l}\operatorname{NL} \phi(v=3) \text { : } \\
\text { isospin breaking in } 1 \mathrm{PE}\left(\propto \in M_{\pi}^{2}\right), \\
\text { contact term without derivatives } \propto \\
\epsilon M_{\pi}^{2}\end{array}$ & - \\
\hline $\begin{array}{l}\mathrm{N}^{3} \mathrm{LO}(v=4) \text { : } \\
\text { subsubleading } 2 \mathrm{PE} \text {, leading } 3 \mathrm{PE} \text {, } \\
\left(1 / m^{2}\right) \text {-corrections to } 1 \mathrm{PE} \text {, } \\
(1 / m) \text {-corrections to } 2 \mathrm{PE} \text {, contact } \\
\text { terms with } 4 \text { derivatives }\end{array}$ & $\begin{array}{l}\operatorname{NNL} \phi(v=4) \text { : } \\
\text { isospin breaking in } 1 \mathrm{PE}\left(\propto e^{2} /\right. \\
\left.(4 \pi)^{2}\right), M_{\pi^{ \pm}} \neq M_{\pi^{0}} \text { in } 2 \mathrm{PE}, \text { tri- } \\
\text { angle and football } 2 \mathrm{PE} \text { diagrams } \\
\propto c_{5}, \pi \gamma \text {-exchange, } m_{n} \neq m_{p} \text { in } \\
2 \mathrm{PE} \text { and in the LS equation, con- } \\
\text { tact terms without derivatives } \propto \\
e^{2} /(4 \pi)^{2}\end{array}$ & - \\
\hline$\cdots$ & $\ldots$ & $\begin{array}{l}\mathrm{N}^{4} \mathrm{~L} \phi(v=6): 1 / m^{2} \text {-corrections } \\
\text { to the static } 1 \gamma \text {-exchange, } 2 \gamma \text { - } \\
\text { exchange. }\end{array}$ \\
\hline
\end{tabular}

\subsubsection{Finite-range isospin-breaking forces}

Let us now give the explicit expressions for the finite-range isospin-violating interactions up to NL $\varnothing$. The dominant $v=2$ contribution ( $\phi$ ) due to $M_{\pi^{ \pm}} \neq M_{\pi^{0}}$ can be taken into account by replacing the isospin-conserving expression $V_{1 \pi}(q)$ in Eq. (2.13) by

$$
\begin{aligned}
& V_{1 \pi, p p}(q)=V_{1 \pi, n n}(q)=-\left(\frac{g_{A}}{2 F_{\pi}}\right)^{2} \vec{\tau}_{1} \cdot \vec{\tau}_{2} \frac{\vec{\sigma}_{1} \cdot \vec{q} \vec{\sigma}_{2} \cdot \vec{q}}{q^{2}+M_{\pi^{0}}^{2}}\left(1-\frac{p^{2}+p^{\prime 2}}{2 m^{2}}\right), \\
& V_{1 \pi, n p, T=1}(q)=-\left(\frac{g_{A}}{2 F_{\pi}}\right)^{2} \vec{\tau}_{1} \cdot \vec{\tau}_{2} \vec{\sigma}_{1} \cdot \vec{q} \vec{\sigma}_{2} \cdot \vec{q}\left(\frac{2}{q^{2}+M_{\pi^{ \pm}}^{2}}-\frac{1}{q^{2}+M_{\pi^{0}}^{2}}\right) \\
& \times\left(1-\frac{p^{2}+p^{\prime 2}}{2 m^{2}}\right), \\
& V_{1 \pi, n p, T=0}(q)=-\left(\frac{g_{A}}{2 F_{\pi}}\right)^{2} \vec{\tau}_{1} \cdot \vec{\tau}_{2} \vec{\sigma}_{1} \cdot \vec{q} \vec{\sigma}_{2} \cdot \vec{q} \frac{1}{q^{2}+M_{\pi}^{2}}\left(1-\frac{p^{2}+p^{\prime 2}}{2 m^{2}}\right),
\end{aligned}
$$

where $T$ denotes the total isospin, $M_{\pi^{ \pm}}$and $M_{\pi^{0}}$ are the masses of the charged and neutral pions, respectively, and

$$
M_{\pi}=\frac{2}{3} M_{\pi^{+}}+\frac{1}{3} M_{\pi^{0}}=138.03 \mathrm{MeV} .
$$

The 1PE potential gets further charge independence and charge symmetry breaking contributions at NL $\varnothing$ and NNL $\varnothing$ due to isospin violating pion-nucleon couplings. The final 
expression for the 1PE potential is then of the kind: $\eta_{i} V_{1 \pi}^{(0)}$, where $\eta_{i}$ are the channeldependent constants: $\eta_{n n} \neq \eta_{n p} \neq \eta_{p p}$. Unfortunately, the actual size of isospin-violating corrections to the pion-nucleon coupling is not well determined at presence [33].

The pion mass difference in the 2PE can be incorporated as outlined in Ref. [48]. It is most convenient to consider the isoscalar and isovector 2PE piece separately,

$$
V_{2 \pi}=V_{2 \pi}^{0}+V_{2 \pi}^{1} \vec{\tau}_{1} \cdot \vec{\tau}_{2}
$$

The isoscalar part $V_{2 \pi}^{0}$ can be expressed as [48]

$$
\begin{aligned}
V_{2 \pi}^{0} & =\frac{2}{3} V_{2 \pi}^{0}\left(M_{\pi^{+}}, M_{\pi^{+}}\right)+\frac{1}{3} V_{2 \pi}^{0}\left(M_{\pi^{0}}, M_{\pi^{0}}\right) \\
& =V_{2 \pi}^{0}\left(M_{\pi}, M_{\pi}\right)+\mathcal{O}\left[\left(\frac{M_{\pi^{+}}-M_{\pi^{0}}}{M_{\pi^{+}}}\right)^{2}\right],
\end{aligned}
$$

where the arguments of $V_{2 \pi}^{0}$ denote the masses of exchanged pions. For the isovector $2 \mathrm{PE}$ $V_{2 \pi}^{1}$, one has the general structure

$$
\begin{aligned}
V_{2 \pi}^{1}= & \tau_{1}^{3} \tau_{2}^{3} V_{2 \pi}^{1}\left(M_{\pi^{+}}, M_{\pi^{+}}\right)+\left(\vec{\tau}_{1} \cdot \vec{\tau}_{2}-\tau_{1}^{3} \tau_{2}^{3}\right) V_{2 \pi}^{1}\left(M_{\pi^{+}}, M_{\pi^{0}}\right) \\
= & \left\{\begin{array}{c}
V_{2 \pi}^{1}\left(M_{\pi^{+}}, M_{\pi^{+}}\right) \quad \text { for } p p \text { and } n n, \\
2 V_{2 \pi}^{1}\left(M_{\pi^{+}}, M_{\pi^{0}}\right)-V_{2 \pi}^{1}\left(M_{\pi^{+}}, M_{\pi^{+}}\right) \sim V_{2 \pi}^{1}\left(M_{\pi^{0}}, M_{\pi^{0}}\right) \\
\quad \text { for } n p, T=1 .
\end{array}\right.
\end{aligned}
$$

The result in the last line of the above equation is valid modulo $\left(\left(M_{\pi^{+}}-M_{\pi^{0}}\right) / M_{\pi^{+}}\right)^{2}-$ corrections. For the $T=0$ case the $2 \mathrm{PE}$ potential reads $V_{2 \pi}\left(M_{\pi}, M_{\pi}\right)$.

The $\pi \gamma$ exchange diagrams have been calculated in Ref. [32] and we give below the results obtained in that paper omitting all computational details. Due to isospin, only charged pion exchange can contribute to the $\pi \gamma$ potential $V_{\pi \gamma}$ and thus it only affects the $n p$ system. The potential has the form

$$
\begin{aligned}
& V_{\pi \gamma}(\vec{q})=-\frac{g_{A}^{2}}{4 F_{\pi}^{2} M_{\pi^{+}}^{2}}\left(\vec{\tau}_{1} \cdot \vec{\tau}_{2}-\tau_{1}^{3} \tau_{2}^{3}\right) \vec{\sigma}_{1} \cdot \vec{q} \vec{\sigma}_{2} \cdot \vec{q} V_{\pi \gamma}(\beta), \\
& V_{\pi \gamma}(\beta)=\frac{\alpha}{\pi}\left[-\frac{\left(1-\beta^{2}\right)^{2}}{2 \beta^{4}\left(1+\beta^{2}\right)} \ln \left(1+\beta^{2}\right)+\frac{1}{2 \beta^{2}}-\frac{2 \bar{\gamma}}{1+\beta^{2}}\right] .
\end{aligned}
$$

Here, $\beta=|\vec{q}| / M_{\pi^{+}}$and $\bar{\gamma}$ is a regularization scheme dependent constant. The analytical form of $V_{\pi \gamma}$ is similar to the one of the 1PE potential, but it differs in strength by the factor $\alpha / \pi \simeq 1 / 400$.

Finally, the expressions for the remaining isospin-violating $2 \mathrm{PE}$ contributions at NNL $\varnothing$ have been given in [35].

\subsubsection{Long-range (soft) isospin-breaking forces}

We now discuss long-range isospin-breaking interactions which are often referred to in the literature as "electromagnetic forces". The static Coulomb force in Eq. (2.43) does certainly not completely represent the electromagnetic interaction between two nucleons but only its leading contribution. The first long-range corrections to the static Coulomb force 
are either suppressed by $m^{-2}$ (relativistic corrections to the static one-photon exchange) or by an additional power of the fine-structure constant $\alpha$ (two-photon exchange). Although all these effects are formally of higher order, we nevertheless prefer to take them into account explicitly for the following reasons. First of all, the effects of these interactions are magnified at low energy due to their long-range nature. Further, as explained above, in our analysis we have to take into account isospin-breaking effects in the same way as it is done in [38]. The electromagnetic interaction for the $p p$ and $n p$ case is given by

$$
\begin{aligned}
& V_{\mathrm{EM}}(p p)=V_{\mathrm{C} 1}+V_{\mathrm{C} 2}+V_{\mathrm{VP}}+V_{\mathrm{MM}}(p p), \\
& V_{\mathrm{EM}}(n p)=V_{\mathrm{MM}}(n p), \\
& V_{\mathrm{EM}}(n n)=V_{\mathrm{MM}}(n n),
\end{aligned}
$$

where $V_{\mathrm{C} 1}$ and $V_{\mathrm{C} 2}$ are usually referred to as "improved Coulomb potential". They take into account the relativistic $\left(1 / \mathrm{m}^{2}\right)$-corrections to the static Coulomb potential and include contributions of the two-photon-exchange diagrams [49]. The explicit coordinate-space expressions read:

$$
\begin{aligned}
& V_{\mathrm{C} 1}=\frac{\alpha^{\prime}}{r}, \\
& V_{\mathrm{C} 2}=-\frac{1}{2 m_{p}^{2}}\left[\left(\Delta+k^{2}\right) \frac{\alpha}{r}+\frac{\alpha}{r}\left(\Delta+k^{2}\right)\right] \sim-\frac{\alpha \alpha^{\prime}}{m_{p} r^{2}},
\end{aligned}
$$

where $\Delta$ denotes the Laplacian. The energy-dependent constant $\alpha^{\prime}$ is given by

$$
\alpha^{\prime}=\alpha \frac{m_{p}^{2}+2 k^{2}}{m_{p} \sqrt{m_{p}^{2}+k^{2}}} .
$$

Here $k$ is the c.m.s. scattering momentum. The term $V_{\mathrm{C} 2}$ is chosen in such a way that it leads to an exact cancellation between the proper two-photon and the iterated onephoton exchange, see [49] for more details. The approximation made in the second line of Eq. (2.53) is based upon using Coulomb distorted-wave Born approximation (CDWBA), see [50] for more details. The modified Coulomb potential $V_{\mathrm{C} 1}$ in Eq. (2.53) can be treated in momentum space in the same way as the usual static Coulomb potential as described in Appendix C. ${ }^{8}$ The magnetic moment interaction $V_{\mathrm{MM}}$ in Eq. (2.52) is given by [51]

$$
\begin{aligned}
& V_{\mathrm{MM}}(p p)=-\frac{\alpha}{4 m_{p}^{2} r^{3}}\left[\mu_{p}^{2} S_{12}+\left(6+8 \kappa_{p}\right) \vec{L} \cdot \vec{S}\right], \\
& V_{\mathrm{MM}}(n p)=-\frac{\alpha \kappa_{n}}{2 m_{n} r^{3}}\left[\frac{\mu_{p}}{2 m_{p}} S_{12}+\frac{1}{m}(\vec{L} \cdot \vec{S}+\vec{L} \cdot \vec{A})\right], \\
& V_{\mathrm{MM}}(n n)=-\frac{\alpha \mu_{n}^{2}}{4 m_{n}^{2} r^{3}} S_{12},
\end{aligned}
$$

\footnotetext{
$\overline{8}$ Clearly, one has to use the appropriately adjusted regular and irregular Coulomb functions $F_{l}(r)$ and $G_{l}(r)$.
} 
where $\mu_{p}=2.793$ and $\mu_{n}=-1.913$ are the proton and neutron magnetic moments and $\kappa_{p}=\mu_{p}-1, \kappa_{n}=\mu_{n}$ their anomalous magnetic moments. Further, $\vec{L}$ is the orbital angular momentum, $\vec{A}=\left(\vec{\sigma}_{1}-\vec{\sigma}_{2}\right) / 2$ and $S_{12}=\left(\vec{\sigma}_{1} \cdot \vec{r}\right)\left(\vec{\sigma}_{2} \cdot \vec{r}\right) / r^{2}-\vec{\sigma}_{1} \cdot \vec{\sigma}_{2} / 3$. The corresponding expressions in momentum space can be found, e.g., in [52]. Finally, the vacuum polarization potential $V_{\mathrm{VP}}$ derived by Ueling [53], see also [54], reads

$$
V_{\mathrm{VP}}=\frac{2 \alpha}{3 \pi} \frac{\alpha^{\prime}}{r} \int_{1}^{\infty} d x e^{-2 m_{e} r x}\left(1+\frac{1}{2 x^{2}}\right) \frac{\left(x^{2}-1\right)^{1 / 2}}{x^{2}},
$$

where $m_{e}$ is the electron mass. Clearly, the vacuum polarization potential is not of an infinitely long range. Its range is governed by the electron mass, which is still tiny compared to the relevant mass scales in the nucleon-nucleon problem. This is similar to the treatment of vacuum polarization in EFT approaches for hadronic bound states, see, e.g., [55].

It is important to realize that the expressions (2.52) refer to point-like nucleons and only define the long-distance asymptotics of the corresponding electromagnetic interaction. The short-distance structure is more complicated and not shown explicitly. In particular, we do not include zero-range (for point-like nucleons) terms as well as electromagnetic form factors which can, in principle, be calculated consistently in EFT. Such short-range terms with the nucleon form factors of a dipole form are, for example, included in the Argonne V18 potential. Last but not least, we note that the above consideration of the electromagnetic effects is based on the "nonrelativistic" Schrödinger equation (3.12), which will be defined in the next section. To close this section let us point out some well-known practical complications which arise due to the presence of the long-range electromagnetic forces.

- Asymptotic states are affected by electromagnetic interactions. The $S$-matrix has to be formulated in terms of asymptotic Coulomb states.

- The formally suppressed (as compared to the strong nuclear force) electromagnetic interactions are enhanced at low energy. The Coulomb interaction requires a nonperturbative treatment. Even the effects due to magnetic moment interaction might be large for certain observables under specific kinematical conditions. For example, in the $n p$ system, it gives rise to a forward-angle dip structure for the analyzing power.

- The expansion of the scattering amplitude in partial waves converges very slowly in the presence of magnetic moment interactions.

\section{Scattering equations}

We start with the relativistic Schrödinger equation (A.4) and assume the potential to be of a finite range. The treatment of the nucleon-nucleon scattering problem in the presence of the long-range Coulomb interaction is relegated to Appendix C. The scattering states are described by the Lippmann-Schwinger equation corresponding to the Schrödinger equation (A.4). The LS equation (for the $T$-matrix) projected onto states with orbital angular momentum $l$, total spin $s$ and total angular momentum $j$ is 


$$
\begin{aligned}
T_{l l^{\prime}}^{s j}\left(p, p^{\prime}\right)= & V_{l l^{\prime}}^{s j}\left(p, p^{\prime}\right)+\sum_{l^{\prime \prime}} \int_{0}^{\infty} \frac{d p^{\prime \prime} p^{\prime \prime 2}}{(2 \pi)^{3}} V_{l l^{\prime \prime}}^{s j}\left(p, p^{\prime \prime}\right) \\
& \times \frac{1}{2 \sqrt{p^{\prime 2}+m^{2}}-2 \sqrt{p^{\prime \prime 2}+m^{2}}+i \eta} T_{l^{\prime \prime} l^{\prime}}^{s j}\left(p^{\prime \prime}, p^{\prime}\right),
\end{aligned}
$$

with $\eta \rightarrow 0^{+}$. In the uncoupled case, $l$ is conserved. The partial wave projected potential $V_{l^{\prime}, l}^{s j}\left(p^{\prime}, p\right)$ can be obtained using the formulae collected in Appendix B. The relation between the $S$ - and on-the-energy shell $T$-matrix is given by

$$
S_{l l^{\prime}}^{s j}(p)=\delta_{l l^{\prime}}-\frac{i}{8 \pi^{2}} p \sqrt{p^{2}+m^{2}} T_{l l^{\prime}}^{s j}(p) .
$$

The phase shifts in the uncoupled cases can be obtained from the $S$-matrix via

$$
S_{j j}^{0 j}=e^{2 i \delta_{j}^{0 j}}, \quad S_{j j}^{1 j}=e^{2 i \delta_{j}^{1 j}},
$$

where we have used the notation $\delta_{l}^{s j}$. Throughout, we use the so-called Stapp parametrization [56] of the $S$-matrix in the coupled channels $(j>0)$ :

$$
\begin{aligned}
S & =\left(\begin{array}{cc}
S_{j-1 j-1}^{1 j} & S_{j-1 j+1}^{1 j} \\
S_{j+1 j-1}^{1 j} & S_{j+1 j+1}^{1 j}
\end{array}\right) \\
& =\left(\begin{array}{cc}
\cos (2 \epsilon) \exp \left(2 i \delta_{j-1}^{1 j}\right) & i \sin (2 \epsilon) \exp \left(i \delta_{j-1}^{1 j}+i \delta_{j+1}^{1 j}\right) \\
i \sin (2 \epsilon) \exp \left(i \delta_{j-1}^{1 j}+i \delta_{j+1}^{1 j}\right) & \cos (2 \epsilon) \exp \left(2 i \delta_{j+1}^{1 j}\right)
\end{array}\right) .
\end{aligned}
$$

For the discussion of the effective range expansion for the ${ }^{3} S_{1}$ partial wave we will use the different parametrization of the $S$-matrix, namely the one due to Blatt and Biedenharn [57]. The connection between these two sets of parameter is given by the following equations:

$$
\begin{gathered}
\delta_{j-1}+\delta_{j+1}=\hat{\delta}_{j-1}+\hat{\delta}_{j+1}, \\
\sin \left(\delta_{j-1}-\delta_{j+1}\right)=\frac{\tan (2 \epsilon)}{\tan (2 \hat{\epsilon})}, \\
\sin \left(\hat{\delta}_{j-1}-\hat{\delta}_{j+1}\right)=\frac{\sin (2 \epsilon)}{\sin (2 \hat{\epsilon})},
\end{gathered}
$$

where $\hat{\delta}$ and $\hat{\epsilon}$ denote the quantities in the Blatt-Biedenharn parametrization and we have omitted the superscripts for $\delta$ 's.

To close this section we would like to remind the reader that the Schrödinger and Lippmann-Schwinger equations (A.4) and (3.1) may be cast into a nonrelativistic form. One way to do that is using the Kamada-Glöckle transformation [58], which relates the relativistic and nonrelativistic c.m.s. momenta $\vec{p}$ and $\vec{q}$ via

$$
T_{\text {kin }}=2 \sqrt{p^{2}+m^{2}}-2 m=\frac{q^{2}}{m} .
$$

The potential $\tilde{V}$ to be used in the nonrelativistic Schrödinger equation

$$
\left[\frac{q^{2}}{m}+\tilde{V}\right] \phi=E \phi,
$$


is defined in the partial-wave projected representation as

$$
\begin{aligned}
\tilde{V}_{l l^{\prime}}^{s j}\left(q, q^{\prime}\right)= & \sqrt{\left(1+\frac{q^{2}}{2 m^{2}}\right) \sqrt{1+\frac{q^{2}}{4 m^{2}}} V_{l l^{\prime}}^{s j}}\left(\sqrt{q^{2}+\frac{q^{4}}{4 m^{2}}}, \sqrt{q^{\prime 2}+\frac{q^{\prime 4}}{4 m^{2}}}\right) \\
& \times \sqrt{\left(1+\frac{q^{\prime 2}}{2 m^{2}}\right) \sqrt{1+\frac{q^{\prime 2}}{4 m^{2}}}}
\end{aligned}
$$

where $V_{l l^{\prime}}^{s j}\left(p, p^{\prime}\right)$ is the potential entering the relativistic Schrödinger equation (A.4). The wave-function $\phi$ is related to $\Psi$ in Eq. (A.4) via

$$
\phi(q)=\sqrt{\left(1+\frac{q^{2}}{2 m^{2}}\right) \sqrt{1+\frac{q^{2}}{4 m^{2}}}} \Psi\left(\sqrt{q^{2}+\frac{q^{4}}{4 m^{2}}}\right) .
$$

The $S$-matrix is defined via

$$
\tilde{S}_{l l^{\prime}}^{s j}(q)=\delta_{l l^{\prime}}-\frac{i}{8 \pi^{2}} q m \tilde{T}_{l l^{\prime}}^{s j}(q)
$$

where the $T$-matrix $\tilde{T}_{l l^{\prime}}^{s j}$ satisfies the usual nonrelativistic Lippmann-Schwinger equation

$$
\tilde{T}_{l l^{\prime}}^{s j}\left(q, q^{\prime}\right)=\tilde{V}_{l l^{\prime}}^{s j}\left(q, q^{\prime}\right)+\sum_{l^{\prime \prime}} \int_{0}^{\infty} \frac{d q^{\prime \prime} q^{\prime \prime 2}}{(2 \pi)^{3}} \tilde{V}_{l l^{\prime \prime}}^{s j}\left(q, q^{\prime \prime}\right) \frac{m}{q^{\prime 2}-q^{\prime \prime 2}+i \eta} \tilde{T}_{l^{\prime \prime} l^{\prime}}^{s j}\left(q^{\prime \prime}, q^{\prime}\right) \text {. }
$$

It can be demonstrated [58] that the $S$-matrix $\tilde{S}_{l l^{\prime}}^{S j}$ equals for any given energy the $S$-matrix $S_{l l^{\prime}}^{s j}$ defined in Eq. (3.2), that is $\tilde{S}_{l l^{\prime}}^{s j}(q)=S_{l l^{\prime}}^{s j}(p)$. Another commonly used way to cast the relativistic Schrödinger equation (A.4) into a nonrelativistic-like form is based upon the algebraic manipulations with this equation, see [25]. More precisely, adding $2 m$ to both sides in Eq. (A.4) with subsequent squaring them, subtracting $4 m^{2}$ and dividing both sides by $4 m$ leads to

$$
\left[\frac{p^{2}}{m}+\bar{V}\right] \Psi=\frac{k^{2}}{m} \Psi
$$

where the momentum $k$ is related to the energy $E$ in Eq. (A.4) via

$$
E=2 \sqrt{k^{2}+m^{2}}-2 m,
$$

and the potential operator $\bar{V}$ is given by

$$
\bar{V}=\left\{\frac{\sqrt{p^{2}+m^{2}}}{2 m}, V\right\}+\frac{V^{2}}{4 m},
$$

or, in the partial-wave projected basis, by 


$$
\begin{aligned}
\bar{V}_{l l^{\prime}}^{s j}\left(p, p^{\prime}\right)= & \left(\frac{\sqrt{p^{2}+m^{2}}}{2 m}+\frac{\sqrt{p^{2}+m^{2}}}{2 m}\right) V_{l l^{\prime}}^{s j}\left(p, p^{\prime}\right) \\
& +\frac{1}{4 m} \sum_{l^{\prime \prime}} \int_{0}^{\infty} \frac{d p^{\prime \prime} p^{\prime \prime 2}}{(2 \pi)^{3}} V_{l l^{\prime \prime}}^{s j}\left(p, p^{\prime \prime}\right) V_{l^{\prime \prime} l^{\prime}}^{s j}\left(p^{\prime \prime}, p^{\prime}\right) .
\end{aligned}
$$

The curly bracket in Eq. (3.14) denote an anticommutator. Notice that contrary to the previously described approach, the "nonrelativistic" Schrödinger equation (3.12) still requires relativistic kinematics in relating the energy and momentum, see Eq. (3.13). The $S$ - and $T$-matrices $\bar{S}_{l l^{\prime}}^{s j}$ and $\bar{T}_{l l^{\prime}}^{s j}$ are defined via Eqs. (3.10) and (3.11), respectively (with $\tilde{S}, \tilde{T}, q$, $q^{\prime}$ being replaced by $\left.\bar{S}, \bar{T}, p, p^{\prime}\right)$. At any given momentum $p$ one has $\bar{S}_{l l^{\prime}}^{s j}(p)=S_{l l^{\prime}}^{s j}(p)$. We have also checked numerically that both equations (3.7) and (3.12) lead to identical results. It should be understood that both ways to cast the relativistic Schrödinger equation (A.4) into a nonrelativistic form discussed in this section are limited to the two-nucleon problem. To the best of our knowledge, no extension to different systems has yet been offered. Consequently, three- and more-nucleon observables calculated using a nonrelativistic approach with the NN potential $\tilde{V}$ or $\bar{V}$ will lead to different results. One should therefore use the relativistic Schrödinger equation (or Faddeev-Yakubovsky equations) with the potential $V$ in such cases. The same applies for processes with external probes.

\section{Bound state}

We now turn to bound state (i.e., deuteron) properties. The deuteron binding energy $E_{\mathrm{d}}$ and wave function $\Psi_{l}^{\mathrm{d}}(p)$ can be obtained from the homogeneous part of Eq. (3.1)

$$
\Psi_{l}^{\mathrm{d}}(p)=\frac{1}{E_{\mathrm{d}}-\left(2 \sqrt{p^{2}+m^{2}}-2 m\right)} \sum_{l^{\prime}} \int_{0}^{\infty} \frac{d p^{\prime} p^{\prime 2}}{(2 \pi)^{3}} V_{l, l^{\prime}}^{s j}\left(p, p^{\prime}\right) \Psi_{l^{\prime}}^{\mathrm{d}}\left(p^{\prime}\right),
$$

with $s=j=1$ and $l=l^{\prime}=0,2$, or, alternatively, from the nonrelativistic-like equation

$$
\Psi_{l}^{\mathrm{d}}(p)=\frac{1}{E_{\mathrm{d}}+E_{\mathrm{d}}^{2} /(4 m)-p^{2} / m} \sum_{l^{\prime}} \int_{0}^{\infty} \frac{d p^{\prime} p^{\prime 2}}{(2 \pi)^{3}} \bar{V}_{l, l^{\prime}}^{s j}\left(p, p^{\prime}\right) \Psi_{l^{\prime}}^{\mathrm{d}}\left(p^{\prime}\right),
$$

where $\bar{V}_{l, l^{\prime}}^{s j}\left(p, p^{\prime}\right)$ is related to $V_{l, l^{\prime}}^{s j}\left(p, p^{\prime}\right)$ via Eq. (3.15). Here we have used the relation (3.13) between the binding energy and momentum. In addition, one can also use the nonrelativistic Schrödinger approach as described in the previous section, which leads to

$$
\phi_{l}^{\mathrm{d}}(p)=\frac{1}{E_{\mathrm{d}}-p^{2} / m} \sum_{l^{\prime}} \int_{0}^{\infty} \frac{d p^{\prime} p^{\prime 2}}{(2 \pi)^{3}} \tilde{V}_{l, l^{\prime}}^{s j}\left(p, p^{\prime}\right) \phi_{l^{\prime}}^{\mathrm{d}}\left(p^{\prime}\right),
$$

where $\tilde{V}_{l, l^{\prime}}^{s j}\left(p, p^{\prime}\right)$ is defined via Eq. (3.8). The wave functions $\phi_{l}^{\mathrm{d}}(p)$ and $\Psi_{l}^{\mathrm{d}}(p)$ are related via Eq. (3.9). 
We will now regard the so-called static properties of the deuteron using the nonrelativistic-like equation (4.2). The latter is fully equivalent to the relativistic equation (4.1) and leads to the same wave function, but has the advantage that one can apply the standard nonrelativistic formulae to study various deuteron properties. We denote by $u(r)$ and $w(r)$ the $S$ - and $D$-wave components of the coordinate space wave function $\Psi_{l}^{\mathrm{d}}(r)$ and by $u(p)$ and $w(p)$ the momentum space representations of $u(r) / r$ and $w(r) / r$ :

$$
u(p)=\frac{2}{\pi} \int_{0}^{\infty} u(r) j_{0}(p r) r d r \quad \text { and } \quad w(p)=\frac{2}{\pi} \int_{0}^{\infty} w(r) j_{0}(p r) r d r .
$$

The wave functions $u$ and $w$ are normalized according to

$$
\int_{0}^{\infty} d p p^{2}\left[u(p)^{2}+w(p)^{2}\right]=\int_{0}^{\infty} d r\left[u(r)^{2}+w(r)^{2}\right]=1
$$

The probability $P_{\mathrm{d}}$ to find the nucleons inside of the deuteron in a $D$-state can be calculated via

$$
P_{\mathrm{d}}=\int_{0}^{\infty} d p p^{2} w(p)^{2}=\int_{0}^{\infty} d r w(r)^{2}
$$

Further, one can compute the deuteron quadrupole moment $Q_{\mathrm{d}}$ and the matter root-meansquare (rms) radius $\sqrt{\left\langle r^{2}\right\rangle_{m}^{\mathrm{d}}}$ through the following equations:

$$
\begin{aligned}
Q_{\mathrm{d}}= & \frac{1}{20} \int_{0}^{\infty} d r r^{2} w(r)[\sqrt{8} u(r)-w(r)] \\
= & -\frac{1}{20} \int_{0}^{\infty} d p\left\{\sqrt{8}\left[p^{2} \frac{d u(p)}{d p} \frac{d w(p)}{d p}+3 p w(p) \frac{d u(p)}{d p}\right]\right. \\
& \left.+p^{2}\left(\frac{d w(p)}{d p}\right)^{2}+6 w(p)^{2}\right\}
\end{aligned}
$$

and

$$
\sqrt{\left\langle r^{2}\right\rangle_{m}^{\mathrm{d}}}=\frac{1}{2}\left[\int_{0}^{\infty} d r r^{2}\left[u(r)^{2}+w(r)^{2}\right]\right]^{1 / 2}
$$

The wave functions $u(r)$ and $w(r)$ behave at large $r$ as

$$
u(r) \sim A_{S} \mathrm{e}^{-\gamma r}, \quad w(r) \sim A_{D} \mathrm{e}^{-\gamma r}\left(1+\frac{3}{\gamma r}+\frac{3}{(\gamma r)^{2}}\right),
$$


where $A_{S}$ and $A_{D}$ are the asymptotic normalization factors of the $S$ - and $D$-states, respectively, and $\gamma=\sqrt{\left|m E_{\mathrm{d}}+E_{\mathrm{d}}^{2} / 4\right|}$. Instead of the quantities $A_{S}$ and $A_{D}$, one often introduces the deuteron normalization $N_{\mathrm{d}}$ and the asymptotic $D / S$ ratio $\eta_{\mathrm{d}}$ according to

$$
N_{\mathrm{d}}^{2}=A_{S}^{2}+A_{D}^{2}, \quad \eta_{\mathrm{d}}=\frac{A_{D}}{A_{S}} .
$$

Not all of the above mentioned deuteron properties are observable and can be measured experimentally. The $D$-state probability $P_{\mathrm{d}}$ is well known to be unobservable [59]. The deuteron electric quadrupole moment corresponds to the quadrupole form factor at $|\vec{q}|=0$, where $\vec{q}$ denotes the momentum transfer. Clearly, the expression (4.7), which gives just the deuteron expectation value of the quadrupole operator $Q_{i j}$

$$
Q_{i j} \equiv \frac{1}{4}\left(3 r_{i} r_{j}-\delta_{i j} r^{2}\right)
$$

is only an approximation to the experimentally measured value for the quadrupole moment, which, i.e., does not take into account two-nucleon currents and relativistic corrections, see, e.g., [60] for more details. A related discussion in the framework of EFT can be found in [61]. The situation is similar with the deuteron matter rms-radius $\sqrt{\left\langle r^{2}\right\rangle_{m}^{\mathrm{d}}}$, which is related to the experimentally measured deuteron charge rms-radius $\sqrt{\left\langle r^{2}\right\rangle_{\mathrm{ch}}^{\mathrm{d}}}$ via [62-64]

$$
\left\langle\left. r^{2}\right|_{\mathrm{ch}} ^{\mathrm{d}}=\left\langle r^{2}\right\rangle_{\mathrm{pt}}^{\mathrm{d}}+\left\langle r^{2}\right\rangle_{\mathrm{ch}}^{p}+\left\langle r^{2}\right\rangle_{\mathrm{ch}}^{n}\right.
$$

where $\sqrt{\left\langle r^{2}\right\rangle_{\text {ch }}^{p}}=0.886(11)$ fm (taking the mean of the three recent values form Refs. [6567] and adding the errors in quadrature) and $\left\langle r^{2}\right\rangle_{\mathrm{ch}}^{n}=-0.113(5) \mathrm{fm}^{2}$ [68] are the proton and neutron ms-radii, respectively, and the "point-nucleon" radius of the deuteron $\left\langle r^{2}\right\rangle_{\mathrm{pt}}^{\mathrm{d}}$ is given by

$$
\left\langle r^{2}\right\rangle_{\mathrm{pt}}^{\mathrm{d}}=\left\langle r^{2}\right\rangle_{m}^{\mathrm{d}}+\left\langle r^{2}\right\rangle_{B}^{\mathrm{d}}
$$

Here $\left\langle r^{2}\right\rangle_{B}^{\mathrm{d}}$ subsumes the "nuclear" effects due to two-body currents as well as relativistic corrections. Notice that while the "point-nucleon" deuteron radius is measurable, the matter radius $\left\langle r^{2}\right\rangle_{m}^{\mathrm{d}}$ is clearly not an observable quantity. In particular, the separate contributions $\left\langle r^{2}\right\rangle_{m}^{\mathrm{d}}$ and $\left\langle r^{2}\right\rangle_{B}^{\mathrm{d}}$ change by a unitary transformation in the two-nucleon system, see [64] for more details. For one specific choice of such a transformation, the effects due to two-nucleon currents in $\left\langle r^{2}\right\rangle_{B}^{\mathrm{d}}$ are estimated to be of the order $\sim 0.016 \mathrm{fm}^{2}$ [64]. Contrary to the previously discussed deuteron quadrupole moment and rms-radius, the asymptotic quantities $A_{S}$ and $A_{D}$ (or, equivalently, $N_{\mathrm{d}}$ and $\eta_{\mathrm{d}}$ ) as well as the deuteron binding energy are observables related to the "pure" nucleon-nucleon system. In particular, the binding energy gives the position of the $N N S$-matrix pole, while the normalization $N_{\mathrm{d}}$ is related to the residue of the pole in the following way, see, e.g., [52,69]

$$
N_{\mathrm{d}}^{2}=\lim _{p \rightarrow k_{\mathrm{d}}} \frac{\left(p-k_{\mathrm{d}}\right)}{8 \pi^{2}} p m \hat{\bar{T}}_{00}^{11}(p) .
$$


Here $k_{\mathrm{d}} \equiv i \gamma$ and $\hat{\bar{T}}_{00}^{11}(p)$ is the $\left(l=l^{\prime}=0\right)$-component of the diagonalized $T$-matrix $\hat{\bar{T}}^{11} \equiv U \bar{T}^{11} U^{-1}$, where

$$
U=\left(\begin{array}{cc}
\cos \hat{\epsilon} & \sin \hat{\epsilon} \\
-\sin \hat{\epsilon} & \cos \hat{\epsilon}
\end{array}\right),
$$

and $\hat{\epsilon}$ is the Blatt and Biedenharn mixing angle [57]. Alternatively, one can rewrite Eq. (4.14) in terms of the Blatt and Biedenharn eigenphase shift $\hat{\delta}_{0}(p)$ as

$$
N_{\mathrm{d}}^{2}=\lim _{p \rightarrow k_{\mathrm{d}}} \frac{2 i\left(p-k_{\mathrm{d}}\right)}{1-i \tan \left[\hat{\delta}_{0}(p)\right]} .
$$

Notice that the $T$-matrix becomes real and the phase shift $\delta_{0}(p)$ imaginary at negative energies. Finally, we point out that the asymptotic $D / S$ ratio $\eta_{\mathrm{d}}$ is given by the negative of the Blatt and Biedenharn mixing angle at the deuteron pole [52]

$$
\eta_{\mathrm{d}}=-\tan \hat{\epsilon}\left(k_{\mathrm{d}}\right) .
$$

Up to now we have discussed the deuteron properties in the context of the nonrelativistic-like equation (4.2). As already pointed out before, one could alternatively use the nonrelativistic equation (4.3). Both schemes are completely equivalent for the two-nucleon system and lead to the same phase shifts and the deuteron binding energy. It is also clear from Eq. (4.17) that the asymptotic $D / S$ ratio $\eta_{\mathrm{d}}$ does not change when one uses Eq. (4.3) instead of Eq. (4.2). On the other hand, the normalization $N_{\mathrm{d}}$ or, equivalently, the asymptotic normalization $A_{S}$ will change. Eq. (4.16) takes the form

$$
\tilde{N}_{\mathrm{d}}^{2}=\lim _{\tilde{p} \rightarrow \tilde{k}_{\mathrm{d}}} \frac{2 i\left(\tilde{p}-\tilde{k}_{\mathrm{d}}\right)}{1-i \tan \left[\hat{\tilde{\delta}}_{0}(\tilde{p})\right]},
$$

if one uses the nonrelativistic Schrödinger equation (4.3). Here, $\tilde{k}_{\mathrm{d}}=i \sqrt{\left|m E_{\mathrm{d}}\right|}$ is the nonrelativistic deuteron binding momentum and $\hat{\tilde{\delta}}_{0}$ is the $S$-wave eigenphase shift calculated using Eq. (3.11). Since both schemes are phase equivalent, one has

$$
\hat{\tilde{\delta}}_{0}(\tilde{p})=\hat{\delta}_{0}\left(\tilde{p} \sqrt{1+\tilde{p}^{2} /\left(4 m^{2}\right)}\right) .
$$

Here we made use of the relation (3.6) between the relativistic and nonrelativistic momenta. We have therefore:

$$
\begin{aligned}
& \tilde{N}_{\mathrm{d}}^{2}=\lim _{\tilde{p} \rightarrow \tilde{k}_{\mathrm{d}}} \frac{2 i\left(\tilde{p}-\tilde{k}_{\mathrm{d}}\right)}{1-i \tan \left[\hat{\delta}_{0}\left(\tilde{p} \sqrt{1+\tilde{p}^{2} /\left(4 m^{2}\right)}\right)\right]} \\
& =\lim _{p \rightarrow k_{\mathrm{d}}} \frac{2 i\left(\sqrt{2 m^{2}\left(\sqrt{1+p^{2} / m^{2}}-1\right)}-\sqrt{2 m^{2}\left(\sqrt{1+k_{\mathrm{d}}^{2} / m^{2}}-1\right)}\right)}{1-i \tan \left[\hat{\delta}_{0}(p)\right]} \\
& \cong N_{\mathrm{d}}^{2}\left(1+\frac{3 E_{\mathrm{d}}}{8 m}\right) \text {. }
\end{aligned}
$$


Here we have again used the relation (3.6) between the relativistic and nonrelativistic momenta. The result in the third line of Eq. (4.20) is valid up to corrections of the order $E_{\mathrm{d}}^{2} / \mathrm{m}^{2}$. It can be rewritten in terms of the asymptotic $S$-wave normalization as follows:

$$
\tilde{A}_{S}=A_{S}\left[1+\left(1+\eta_{\mathrm{d}}^{2}\right) \frac{3 E_{\mathrm{d}}}{16 m}\right] .
$$

To end this section, we note that the other deuteron properties such as $P_{\mathrm{d}}$ in Eq. (4.6), $Q_{\mathrm{d}}$ in Eq. $(4.7)^{9}$ and $\sqrt{\left\langle r^{2}\right\rangle_{m}^{\mathrm{d}}}$ in Eq. (4.8), which are not observable, are expected to change when calculated using the nonrelativistic wave function from Eq. (4.3). For a recent reviews on the deuteron the reader is referred to Refs. [70,71].

\section{The fits}

In this section we discuss the determination and specify the values of the various LECs adopted in the present analysis. Throughout this work, we use the following values for the pion decay constant $F_{\pi}$, the pion masses $M_{\pi^{ \pm}}, M_{\pi^{0}}$ and the proton and neutron masses $m_{p}$ and $m_{n}: F_{\pi}=92.4 \mathrm{MeV}, M_{\pi^{ \pm}}=139.5702 \mathrm{MeV}, M_{\pi^{0}}=134.9766 \mathrm{MeV}$, $m_{p}=938.2720 \mathrm{MeV}, m_{n}=939.5653 \mathrm{MeV}$.

For the 1PE potential, we use the expression (2.13) with $g_{A}=1.29$. This larger value of the LEC $g_{A}$ as compared to the standard one $g_{A}=1.26$ is in order to account for the Goldberger-Treiman discrepancy as discussed in Section 2.3. Notice further that we take into account the pion mass difference as given in Eq. (2.46). The leading 2PE potential given in Eq. (2.14) is parameter-free. The NNLO and $\mathrm{N}^{3} \mathrm{LO} 2 \mathrm{PE}$ contributions in Eqs. (2.15), (2.18)-(2.20) depend on the LECs $c_{1}, c_{2}, c_{3}$ and $c_{4}$ from the second-order $\pi N$ Lagrangian as well as on $\bar{d}_{1}+\bar{d}_{2}, \bar{d}_{3}, \bar{d}_{5}$ and $\bar{d}_{14}-\bar{d}_{15}$ from the third-order $\pi N$ Lagrangian. For the LECs $c_{1,4}$ we adopt the central values from the $Q^{3}$-analysis of the $\pi N$ system [73]: $c_{1}=-0.81 \mathrm{GeV}^{-1}, c_{4}=3.40 \mathrm{GeV}^{-1}$. For the constant $c_{3}$ the value $c_{3}=-3.40 \mathrm{GeV}^{-1}$ is used, which is on the lower side but still consistent with the results from reference [73]: $c_{3}=-4.69 \pm 1.34 \mathrm{GeV}^{-1}$. The same value for $c_{3}$ has been adopted in our NNLO analysis [21]. Further, this value was found in Ref. [26] to be consistent with empirical NN phase shifts as well as the results from dispersion and conventional meson theories. Notice, however, that it is about $25 \%$ smaller in magnitude than the value extracted from the partial wave analysis of the $p p$ and $n p$ data [72]. The LEC $c_{2}$ could not be fixed accurately analyzing pion-nucleon scattering inside the Mandelstam triangle in [73]. We therefore adopt the central value found in the third-order analysis [23]: $c_{2}=3.28 \mathrm{GeV}^{-1}$. For the combinations of $d_{i}$ 's, we again use the values found in [23]: $\bar{d}_{1}+\bar{d}_{2}=3.06 \mathrm{GeV}^{-2}, \bar{d}_{3}=-3.27 \mathrm{GeV}^{-2}$, $\bar{d}_{5}=0.45 \mathrm{GeV}^{-2}$ and $\bar{d}_{14}-\bar{d}_{15}=-5.65 \mathrm{GeV}^{-2}$.

\footnotetext{
9 It would be more appropriate to introduce a special notation for the quadrupole moment defined in Eq. (4.7) using nonrelativistic impulse approximation in a way similar to the deuteron rms-radius. Unfortunately, no such notation appears in the literature, which might lead to a confusion. It should be understood that while the deuteron quadrupole moment represents the response of the deuteron to an external electromagnetic field and is certainly measurable, Eq. (4.7) gives only an approximation, which is model-dependent and not observable.
} 
We now turn to short-range contact interactions. The two $v=0$ LECs $C_{S, T}$, seven $v=2$ LECs $C_{1 \ldots 7}$ as well as fifteen $v=4$ LECs $D_{1 \ldots 15}$ in Eq. (2.4) are unknown and have to be fixed from a fit to data (i.e., to Nijmegen phase shifts). Contributions of the contact interactions to various partial waves are given in Eq. (2.5). Thus, we have to determine 8 LECs in the ${ }^{3} S_{1}-{ }^{3} D_{1}$ channel, 4 LECs in the ${ }^{1} S_{0}$ channel, 3 LECs in the ${ }^{3} P_{2}-{ }^{3} F_{2}$ channel, 2 LECs in each of the ${ }^{1} P_{1},{ }^{3} P_{1},{ }^{3} P_{0}$ partial waves and 1 LEC in each of the ${ }^{1} D_{2}$, ${ }^{3} D_{2},{ }^{3} D_{3}-{ }^{3} G_{3}$ channels. In addition to the above mentioned isospin-conserving contact interactions, we have two isospin-violating contact terms with unknown coefficients, see Eqs. (2.35) and (2.37). Both terms contribute to the ${ }^{1} S_{0}$ partial wave and provide chargedependent contributions to the LEC $\tilde{C}_{{ }^{1}}$. In the following, we will therefore distinguish between $\tilde{C}_{1}^{p p}, \tilde{C}_{{ }_{1} S_{0}}^{n p}$ and $\tilde{C}_{1}^{n n}$. We also note that we always use the proper kinematics as given in Appendix A. Let us now specify precisely our way of fixing the LECs. The LECs contributing to isovector channels ${ }^{3} P_{2}-{ }^{3} F_{2},{ }^{3} P_{1},{ }^{3} P_{0}$ and ${ }^{1} D_{2}$ have been fixed from a fit to Nijmegen $p p$ phase shifts [38], which are much more precise than the corresponding $n p$ phase shifts. The isovector $n p$ phase shifts are then extracted from the $p p$ ones in a parameter-free way by taking into account the proper 1PE potential and switching off the electromagnetic interaction. This is precisely the same procedure as used in the Nijmegen PWA [38]. In the ${ }^{1} S_{0}$ partial wave we have to take into account isospin-violating contact interactions as discussed above. We determine the LECs $\tilde{C}_{1}^{p p}, \tilde{C}_{{ }_{1} S_{0}}^{n p}, C^{{ }_{1}} S_{0}, D^{1} S_{0}$ and $D^{2} S_{0}$ from a combined fit in the ${ }^{1} S_{0} p p$ and $n p$ channels. The LEC $\tilde{C}_{1}^{n n}$ is then obtained from the requirement to reproduce the experimental value [74,75] $a_{\mathrm{nn}}=-18.9 \mathrm{fm}$ for the $n n$ scattering length. All remaining LECs are fixed from a fit to $n p$ phases from Nijmegen PWA [38]. We notice that contrary to our NLO and NNLO analysis [16,21], we had to use here a large energy interval, i.e., up to $E_{\text {lab }}=200 \mathrm{MeV}$, in order to fix the LECs. This is because of two reasons: first, the phase shifts in the ${ }^{1} S_{0}$ and in the ${ }^{3} S_{1}-{ }^{3} D_{1}$ channels simply do not show enough structure beyond $E_{\text {lab }}=100 \mathrm{MeV}$ in order to fix reliably 4 and 8 parameters, respectively. Secondly, phase shifts at low energy are not very sensitive to higher-order contact interactions except maybe in the two $S$-waves.

It remains to specify the values for the cut-offs $\Lambda$ and $\tilde{\Lambda}$ which enter the LippmannSchwinger equation and the spectral-function representation of the two-pion exchange potential, respectively. Certainly, both cut-offs are introduced in order to remove highmomentum components of the interacting nucleon and pion fields, which are beyond the range of applicability of the chiral EFT. We remind the reader that from the formal point of view, one can choose any value for the SFR cut-off which is large enough so that the relevant physics is still present. Even the choice $\tilde{\Lambda}=\infty$, which is equivalent to dimensional regularization, is formally possible since all terms with positive powers of $\tilde{\Lambda}$ (and $\propto \ln \tilde{\Lambda}$ ) can be absorbed by redefinition of the corresponding LECs. It has been argued in [21], however, that the choice $\tilde{\Lambda}=500-700 \mathrm{MeV}$ leads to a natural separation of the longand short-range parts of the nuclear force and allows to improve the convergence of the low-momentum expansion. In the present analysis we use this range for $\tilde{\Lambda}$.

While $\tilde{\Lambda}$ is related to perturbative renormalization of the pion loop integrals, the cutoff $\Lambda$ specifies the way of nonperturbative renormalization of the Lippmann-Schwinger equation. Contrary to the SFR cut-off $\tilde{\Lambda}$, one, in general, cannot arbitrarily increase the value of $\Lambda$ [76-79]. This is because one needs an infinite number of counter terms in 
order to absorb all divergences arising through iteration of the potential in the LippmannSchwinger equation. ${ }^{10}$ Keeping $\Lambda$ finite and of the order of the separation scale in the NN problem, one expects the contribution of the higher-order counter terms to be suppressed by powers of the generic low-momentum scale provided that the corresponding LECs are of natural size [76,77]. In our previous NLO and NNLO analyses [81] based on the dimensionally regularized expressions for the potential we have used $\Lambda=500-600 \mathrm{MeV}$ with the regulator function being defined as $f^{\Lambda}(p)=\exp \left[-p^{4} / \Lambda^{4}\right]$. In the more recent study [21] based on the SFR approach we have increased this range to $\Lambda=450-650 \mathrm{MeV}$ using $f^{\Lambda}(p)=\exp \left[-p^{6} / \Lambda^{6}\right]$. We have, however, found in [21], that the upper values of $\Lambda$ are already rather close to its critical value $\Lambda^{c}$, above which one encounters spurious deeplybound states. Notice that the values of various LECs start to strongly vary for $\Lambda \sim \Lambda^{c}$ leaving the natural range. In order to avoid such a situation we slightly reduce the range of variation of $\Lambda$ to $450-600 \mathrm{MeV}$ in the present analysis. To be more specific, we will use the following cut-off combinations (all values in $\mathrm{MeV}$ ):

$$
\{\Lambda, \tilde{\Lambda}\}=\{450,500\},\{600,600\},\{450,700\},\{600,700\} .
$$

For $\tilde{\Lambda}=500 \mathrm{MeV}$ the value $\Lambda=600 \mathrm{MeV}$ is already found to be close to $\Lambda^{c}$. We therefore replace the cut-off combination $\{600,500\}$ by $\{600,600\}$. Notice that further reducing of the $\Lambda$-values beyond $\Lambda=450 \mathrm{MeV}$ is, in principle, possible but leads to a strong increase of the theoretical uncertainty. We therefore refrain from doing that. Finally, we notice that a more elegant regularization prescriptions, like, e.g., lattice regularization, would allow to regularize pion loop integrals and the Lippmann-Schwinger equation in the same way without introducing two independent scales $\Lambda$ and $\tilde{\Lambda}$. For a related recent discussion of the role and optimal choice of the cut-off $\Lambda$ in the LS equation the reader is referred to Refs. [77,79].

Let us now give the precise definition of the phase shifts considered in the present work and remind the reader on the type of phase shifts used in the Nijmegen PWA [38]. We will adopt here the notation of Ref. [50] and denote by $\delta_{W}^{V}$ the phase shift generated by the potential $W$ with respect to the solution with $V$ as the interaction.

\section{1. pp phases}

The full phase shifts $\delta_{\mathrm{EM}+\mathrm{N}}$ of electromagnetic plus strong interaction can be expressed as

$$
\delta_{\mathrm{EM}+\mathrm{N}}=\delta_{\mathrm{EM}}+\delta_{\mathrm{EM}+\mathrm{N}}^{\mathrm{EM}} .
$$

\footnotetext{
10 It has been shown in [80] that $1 / r^{n}$ singular potentials, which arise, e.g., from pion exchange contributions, can be renormalized by a one-parameter square-well counterterm, see [8] for a related work. Although the authors of [80] have demonstrated that the low-energy NN observables can be made independent of the square-well width by adjusting the square-well strength, the power counting scheme adopted in the present work is not consistent with such an approach.
} 
The above expression applies to uncoupled channels. For coupled channels one has to translate the addition law for the phase shifts into a multiplication law for the corresponding $S$-matrices

$$
S_{\mathrm{EM}+\mathrm{N}}=\left(S_{\mathrm{EM}}\right)^{1 / 2} S_{\mathrm{EM}+\mathrm{N}}^{\mathrm{EM}}\left(S_{\mathrm{EM}}\right)^{1 / 2},
$$

see $[51,82]$ for further details. Such a modification for coupled channels will, however, not change the conclusions of this section. We will therefore not consider the coupled case in detail in what follows. The last term in Eq. (5.2) corresponds to the phase shifts of the electromagnetic plus nuclear interaction with respect to electromagnetic wave functions. These phase shifts $\delta_{\mathrm{EM}+\mathrm{N}}^{\mathrm{EM}}$ are the ones which are given in the Nijmegen PWA [38]. Notice that the electromagnetic phase shift $\delta_{\mathrm{EM}}$ can be represented as

$$
\delta_{\mathrm{EM}}=\delta_{\mathrm{C} 1}+\delta_{\mathrm{C} 1+\mathrm{C} 2}^{\mathrm{C} 1}+\delta_{\mathrm{C} 1+\mathrm{C} 2+\mathrm{MM}}^{\mathrm{C} 1+\mathrm{C} 2}+\delta_{\mathrm{C} 1+\mathrm{C} 2+\mathrm{MM}+\mathrm{VP}}^{\mathrm{C} 1+\mathrm{C} 2+\mathrm{MM}}=\delta_{\mathrm{C} 1}+\rho+\phi+\tau,
$$

where we have introduced the abbreviations $\rho \equiv \delta_{\mathrm{C} 1+\mathrm{C} 2}^{\mathrm{C} 1}, \phi \equiv \delta_{\mathrm{C} 1+\mathrm{C} 2+\mathrm{MM}}^{\mathrm{C} 1+\mathrm{C} 2}$ and $\tau \equiv$ $\delta_{\mathrm{C} 1}^{\mathrm{C} 1+\mathrm{C} 2}+\mathrm{MM}+\mathrm{MP}$. In practice, the quantities $\rho, \phi$ and $\tau$ are usually calculated using the CDWBA. This is justified due to the smallness of the corresponding interactions $V_{\mathrm{C} 2}, V_{\mathrm{MM}}$ and $V_{\mathrm{VP}}$. In this case one has approximately

$$
\phi \sim \delta_{\mathrm{C} 1+\mathrm{MM}}^{\mathrm{C} 1}, \quad \tau \sim \delta_{\mathrm{C} 1+\mathrm{VP}}^{\mathrm{C} 1}
$$

For more details the reader is referred to Refs. [50,51].

The phase shifts $\delta$ EM $+\mathrm{N}$ obtained in the Nijmegen PWA do, however, not correspond to the type of phase shifts, which is usually considered in practical calculations, namely the phase shifts $\delta_{\mathrm{C} 1+\mathrm{N}}^{\mathrm{C} 1}$ of the modified Coulomb plus strong interactions with respect to the phase shifts of the modified Coulomb potential. These phase shifts can easily be calculated for any given nuclear potential using, e.g., the method described in Appendix C. The $p p$ phases considered in the present work are of that type. We therefore need to relate $\delta_{\mathrm{C} 1+\mathrm{N}}^{\mathrm{Cl}}$ to the previously discussed phase shifts $\delta_{\mathrm{EM}+\mathrm{N}}^{\mathrm{EM}}$. This can be done by noting that the total phase shift $\delta_{\mathrm{EM}+\mathrm{N}}$ can be expressed in the form

$$
\delta_{\mathrm{EM}+\mathrm{N}}=\delta_{\mathrm{C} 1}+\delta_{\mathrm{C} 1+\mathrm{C} 2+\mathrm{MM}+\mathrm{VP}+\mathrm{N}}^{\mathrm{C} 1} .
$$

In the coupled case one has to modify this relation in a way analogous to Eq. (5.3). Again, due to the smallness of the potentials $V_{\mathrm{C} 2}, V_{\mathrm{MM}}$ and $V_{\mathrm{VP}}$ one can make use of the DWBA

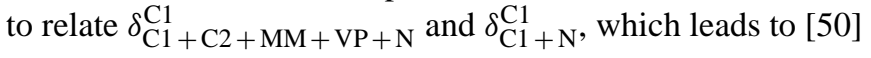

$$
\begin{aligned}
& \left(\delta_{\mathrm{C} 1}^{\mathrm{C} 1}+\mathrm{C} 2+\mathrm{MM}+\mathrm{VP}+\mathrm{N}\right)_{l}-\left(\delta_{\mathrm{C} 1+\mathrm{N}}^{\mathrm{C} 1}\right)_{l} \\
& \equiv \tilde{\Delta}_{l}=\left(\delta_{\mathrm{C} 1+\mathrm{N}+\mathrm{C} 2+\mathrm{MM}+\mathrm{VP})_{l}}^{\mathrm{C}+\mathrm{N}}\right. \\
& \quad \sim \frac{-m_{p}}{k} \int_{0}^{\infty} d r \chi_{l}(r)\left[V_{\mathrm{C} 2}(r)+V_{\mathrm{MM}}(r)+V_{\mathrm{VP}}(r)\right] \chi_{l}(r),
\end{aligned}
$$

where $l$ is the angular momentum and $\chi_{l}(r)$ is the wave function for the potential $V_{\mathrm{C} 1}+V_{\mathrm{N}}$. Combining now Eqs. (5.2)-(5.7) we end up with the following formula which relates the 
phase shifts $\delta_{\mathrm{C} 1+\mathrm{N}}^{\mathrm{C} 1}$ we are calculating to the ones $\delta_{\mathrm{EM}+\mathrm{N}}^{\mathrm{EM}}$ of the Nijmegen PWA:

$$
\left(\delta_{\mathrm{C} 1+\mathrm{N}}^{\mathrm{C} 1}\right)_{l} \sim\left(\delta_{\mathrm{EM}+\mathrm{N}}^{\mathrm{EM}}\right)_{l}-\tilde{\Delta}_{l}+\rho_{l}+\phi_{l}+\tau_{l}
$$

This formula can be further simplified if one notes that for all $l \geqslant 1$ the wave functions $\chi_{l}(r)$ near the threshold are almost not affected by the nuclear interaction [50]. Thus, one can approximately replace in Eq. (5.7) the wave functions $\chi_{l}(r)$ by the regular Coulomb wave functions, which leads to $\tilde{\Delta}_{l} \sim \rho_{l}+\phi_{l}+\tau_{l}$. Therefore, for all partial waves except ${ }^{1} S_{0}$ one has: $\left(\delta_{\mathrm{C} 1+\mathrm{N}}^{\mathrm{C} 1}\right)_{l} \sim\left(\delta_{\mathrm{EM}+\mathrm{N}}^{\mathrm{EM}}\right)_{l}$. In the case of the ${ }^{1} S_{0}$ partial wave, it has been argued based on explicit calculations that the quantity $\tilde{\Delta}_{0}$ is "sufficiently model-independent" for a wide range of nuclear forces at least in the case $V_{\mathrm{C} 2}=0$. Notice that the magnetic moment interaction in Eq. (2.55) does not contribute in that and all other spin-singlet channels. The values of $\tilde{\Delta}_{0}$ based on the Nijmegen N78 potential [83] as well as for the $\tau_{0}$ and $\rho_{0}$ are given in [50] at various energies. In the present analysis, we will use these values for the above mentioned quantities in order to relate our ${ }^{1} S_{0}$ phase shift to the one of the Nijmegen PWA via Eq. (5.8). ${ }^{11}$ Notice that a more accurate way to determine the phase shifts $\left(\delta_{\mathrm{C} 1+\mathrm{N}}^{\mathrm{C} 1}\right)_{l}$ would be to define the regularized expressions for electromagnetic interactions and to calculate the quantities $\rho, \phi, \tau$ and $\tilde{\Delta}_{l}$ explicitly (one can still use DWBA). We, however, believe that there is no need for such a refinement at the level of accuracy of $\mathrm{N}^{3} \mathrm{LO}$.

Let us now summarize our way of calculating phase shifts in the $p p$ system. We compute the phase shifts $\delta_{\mathrm{C} 1+\mathrm{N}}^{\mathrm{C} 1}$ of the modified Coulomb plus nuclear potential $V_{\mathrm{C} 1}+V_{\mathrm{N}}$ with respect to wave functions of the $V_{\mathrm{C} 1}$-potential. The strong interaction part of the chiral nuclear force at order $\mathrm{N}^{3} \mathrm{LO}$ is discussed in Section 2. It has to be used in the relativistic Schrödinger Eq. (A.4), while the electromagnetic interactions in Eq. (2.52), including the modified Coulomb force $V_{\mathrm{C} 1}$, are to be used in the nonrelativistic-like Eq. (3.12). We therefore first apply Eq. (3.15) to derive the modified strong potential for use in Eq. (3.12). ${ }^{12} \mathrm{We}$ then calculate phase shifts $\delta_{\mathrm{C} 1+\mathrm{N}}^{\mathrm{C} 1}$ in momentum space as described in Appendix C. In the fitting procedure the calculated phase shifts $\delta_{\mathrm{C} 1+\mathrm{N}}^{\mathrm{C} 1}$ are compared with the phases $\delta_{\mathrm{EM}+\mathrm{N}}^{\mathrm{EM}}$ of the Nijmegen PWA [38]. For all partial waves except ${ }^{1} S_{0}$ we use the approximation $\left(\delta_{\mathrm{C} 1+\mathrm{N}}^{\mathrm{C} 1}\right)_{l} \sim\left(\delta_{\mathrm{EM}+\mathrm{N}}^{\mathrm{EM}}\right)_{l}$. For the ${ }^{1} S_{0}$ phase, we make use of Eq. (5.8), where the quantities $\tilde{\Delta}_{l}, \rho_{l}$ and $\tau_{l}$ are taken from Ref. [50].

\footnotetext{
11 The quantity $\tilde{\Delta}_{l}$ in [50] does not contain the contribution due to magnetic moment interaction, which has been neglected in that work. In our analysis we only need to know $\tilde{\Delta}_{l}$ explicitly for $l=0$ and therefore can still use the result of [50].

12 Notice that in principle, the modified potential should contain contributions due to electromagnetic interactions. Eq. (3.15) should actually be applied to the sum of the strong interaction and electromagnetic potentials $V_{\mathrm{N}}$ and $V_{\mathrm{em}}$. The modified potential would then contain pieces $\propto V_{\mathrm{N}}, V_{\mathrm{em}},(1 / m) V_{\mathrm{N}} V_{\mathrm{N}},(1 / m) V_{\mathrm{N}} V_{\mathrm{em}}$ and $(1 / m) V_{\mathrm{em}} V_{\mathrm{em}}$. Applying Eq. (3.15) only to the strong potential $V_{\mathrm{N}}$ and adding the appropriate (modified) electromagnetic contributions, we thus miss terms $\propto(1 / m) V_{\mathrm{N}} V_{\mathrm{em}}$. Such contributions are suppressed by a factor $Q / m$ compared to the $\pi \gamma$-exchange in Eq. (2.51) and beyond the accuracy of the present calculation.
} 


\section{2. np phases}

As already pointed out in Section 2.4.2, the electromagnetic interaction in the $n p$ case is given entirely in terms of magnetic moment interaction $V_{\mathrm{MM}}(n p)$. Consequently, Eq. (5.2) takes the form

$$
\delta_{\mathrm{MM}+\mathrm{N}}=\delta_{\mathrm{MM}}+\delta_{\mathrm{MM}+\mathrm{N}}^{\mathrm{MM}} .
$$

The $n p$ phase shifts of the Nijmegen PWA as well as in our analysis correspond to the phase shifts $\delta_{\mathrm{MM}+\mathrm{N}}^{\mathrm{MM}}$ of nuclear plus magnetic moment interactions with respect to magnetic moment interaction wave functions. Notice that the term in $V_{\mathrm{MM}}(n p)$ in Eq. (2.55) proportional to $\vec{A}$ gives rise to the so-called "class IV" isospin-breaking force [84], which mixes spin-singlet and spin-triplet states. The contribution of this term is very small and usually only taken into account when constructing the magnetic moment scattering amplitude, see, e.g., [85]. In our analysis we make use of the standard approximation [85]

$$
\delta_{\mathrm{MM}+\mathrm{N}}^{\mathrm{MM}} \sim \delta_{\mathrm{N}}
$$

for all $(l \neq 0)$-states. Therefore and because of the fact that the magnetic moment interaction does not contribute to the ${ }^{1} S_{0}$ channel, we have to take into account $V_{\mathrm{MM}}(\mathrm{np})$ explicitly only in the ${ }^{3} S_{1}-{ }^{3} D_{1}$ partial wave. In that case the phase shift $\delta_{\mathrm{MM}+\mathrm{N}}^{\mathrm{MM}}$ is calculated by subtracting $\delta_{\mathrm{MM}}$ from $\delta_{\mathrm{MM}+\mathrm{N}}$, where the phase shifts $\delta_{\mathrm{MM}}$ are obtained using the Born approximation.

\section{3. nn phases}

As in the previously considered case of neutron-proton scattering, in the $n n$ system one has to take into account only magnetic moment interaction. Decomposing the phase shifts as in Eq. (5.9) one can make use of the approximation $\delta_{\mathrm{MM}+\mathrm{N}}^{\mathrm{MM}} \sim \delta_{\mathrm{N}}$ in all $n n$ partial waves. As already stated before, this approximation is accurate for partial waves with $l \neq 0$. In the case of the ${ }^{1} S_{0}$ partial wave, the phase shift is still given by $\delta_{\mathrm{N}}$ since the long-range magnetic moment interaction does not contribute to this channel. Therefore, $n n$ phase shifts in all partial waves correspond to $\delta_{\mathrm{N}}$.

\section{Results and discussion}

\subsection{Phase shifts}

In the following sections, we will show the results for the $n p$ phase shifts. Before showing the results of our analysis, let us make a simple estimate for the expected theoretical uncertainty at $\mathrm{N}^{3} \mathrm{LO}$. Following the reasoning of Ref. [21], we expect for the uncertainty of a scattering observable at c.m.s. momentum $k$ at $\mathrm{N}^{3} \mathrm{LO}$ to be of the or$\operatorname{der} \sim\left(\max \left[k, M_{\pi}\right] / \lambda\right)^{5}$. To provide a fair estimate, we identify the hard scale $\lambda$ with the smallest value of the ultraviolet cut-off, i.e., we adopt $\lambda \sim 450 \mathrm{MeV}$. This results in the following estimations for the theoretical (maximal) uncertainty: 


$$
\begin{aligned}
& \sim 0.5 \% \text { at } E_{\text {lab }} \sim 50 \mathrm{MeV} \text { and below, } \\
& \sim 7 \% \text { at } E_{\text {lab }} \sim 150 \mathrm{MeV}, \\
& \sim 25 \% \text { at } E_{\text {lab }} \sim 250 \mathrm{MeV} .
\end{aligned}
$$

One should keep in mind that the above estimations are fairly rough. For a detailed discussion on the theoretical uncertainty, especially at NLO and NNLO, the reader is referred to [21].

\subsubsection{S-waves}

The phase shifts in the ${ }^{1} S_{0}$ and ${ }^{3} S_{1}$ partial waves are shown in Fig. 3. Both are visibly improved compared to the NNLO result. For $E_{\text {lab }}=50 \mathrm{MeV}, 150 \mathrm{MeV}$ and $250 \mathrm{MeV}$ we find the phase shift in the ${ }^{1} S_{0}$ partial wave in the ranges $40.42^{\circ}-40.72^{\circ}, 16.04^{\circ}-17.03^{\circ}$ and $2.22^{\circ}-4.76^{\circ}$, respectively. These values agree well with the ones from the Nijmegen PSA: $\delta=40.54^{\circ}, \delta=16.94^{\circ}$ and $\delta=1.96^{\circ}$. The relative uncertainty of our results is in agreement with the above estimations except for $E_{\text {lab }}=250 \mathrm{MeV}$, where the phase shift is

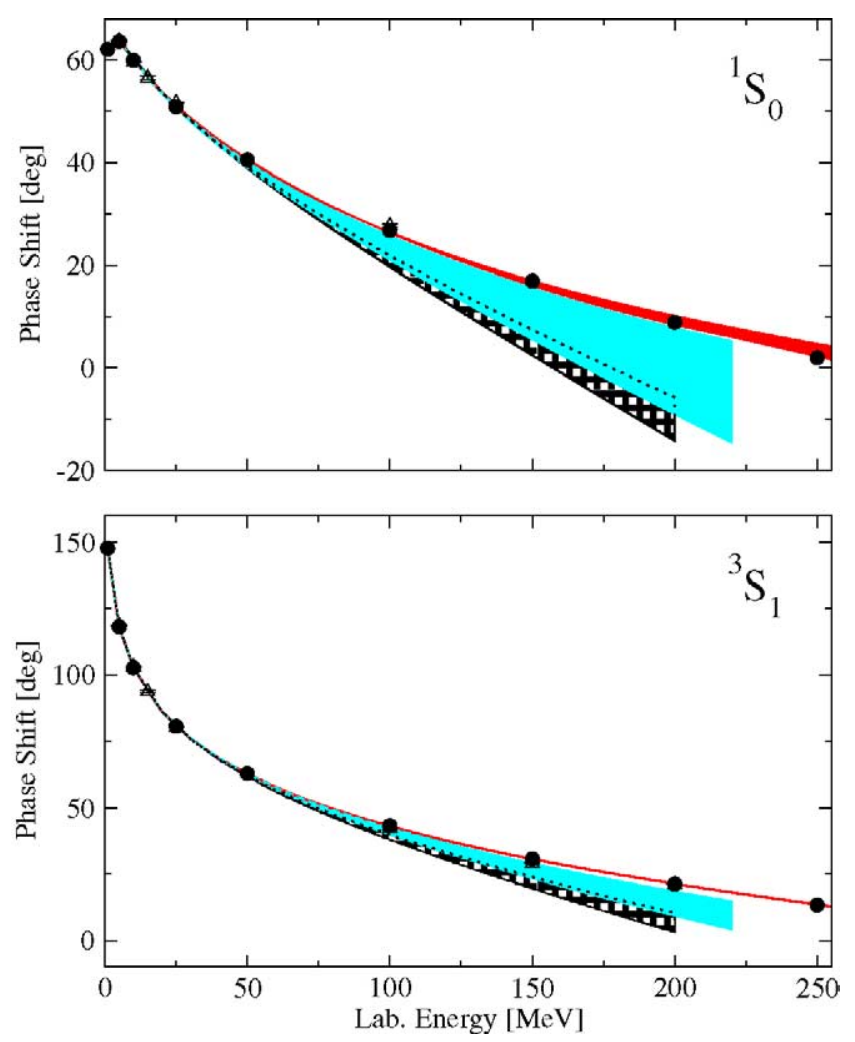

Fig. 3. $S$-wave $n p$ phase shifts versus the nucleon laboratory energy. The grid, light shaded and dark shaded bands show the NLO, NNLO [21] and $\mathrm{N}^{3}$ LO results, respectively. The cut-offs $\Lambda$ and $\tilde{\Lambda}$ at $\mathrm{N}^{3} \mathrm{LO}$ are varied as specified in Eq. (5.1). The filled circles depict the Nijmegen PWA results [38] and the open triangles are the results from the Virginia Tech PWA [106]. 
Table 2

The $S$-wave LECs $\tilde{C}_{i}, C_{i}$ and $D_{i}$ at $\mathrm{N}^{3} \mathrm{LO}$ for the different cut-off combinations $\{\Lambda[\mathrm{MeV}], \tilde{\Lambda}[\mathrm{MeV}]\}$. The values of the $\tilde{C}_{i}$ are in $10^{4} \mathrm{GeV}^{-2}$, of the $C_{i}$ in $10^{4} \mathrm{GeV}^{-4}$ and of the $D_{i}$ in $10^{4} \mathrm{GeV}^{-6}$

\begin{tabular}{lrrrr}
\hline LEC & $\{450,500\}$ & $\{600,600\}$ & $\{450,700\}$ & $\{600,700\}$ \\
\hline$\tilde{C}_{1}^{\mathrm{pp}} S_{0}$ & -0.0834 & -0.0800 & -0.1247 & -0.0436 \\
$\tilde{C}_{1}^{\mathrm{np}} S_{0}$ & -0.0913 & -0.0892 & -0.1289 & -0.0544 \\
$\tilde{C}_{1}^{\mathrm{nn}} S_{0}$ & -0.0880 & -0.0851 & -0.1272 & -0.0494 \\
$C_{1} S_{0}$ & 1.5007 & 1.8075 & 2.1217 & 1.8950 \\
$D^{1} S_{0}$ & -26.9836 & -16.7678 & -24.7288 & -17.6295 \\
$D^{2} S_{0}$ & 3.7402 & -2.5565 & 0.8214 & -2.0771 \\
$\tilde{C}_{3} S_{1}$ & -0.1498 & 0.1782 & -0.1599 & 0.0746 \\
$C_{3} S_{1}$ & 0.4144 & -0.9058 & 0.6275 & -0.3557 \\
$D_{3}^{1} S_{1}$ & -26.3516 & -13.4902 & -23.8555 & -12.4078 \\
$D_{3}^{2} S_{1}$ & 4.8091 & 2.6661 & 4.3807 & 1.8895 \\
\hline
\end{tabular}

close to 0 . The results for the ${ }^{3} S_{1}$ partial wave are similar to the ones in the ${ }^{1} S_{0}$ channel. The uncertainty due to the cut-off variation is found to be smaller in this case.

The pertinent $S$-wave LECs are tabulated in Table 2 for the four pairs of cut-offs (5.1). Here, several remarks are in order. First, we note that, in general, one has to expect multiple solutions for the LECs. This problem has already been discussed in [16] at NLO and NNLO. For the ${ }^{1} S_{0}$ channel, we have to fix five LECs $\tilde{C}_{{ }^{p} S_{0}}^{p p}, \tilde{C}^{n p}{ }_{S_{0}}^{n p}, C_{1} S_{0}, D_{1}^{1} S_{0}, D_{1}^{2} S_{0}$, from a fit to Nijmegen $p p$ and $n p$ phase shifts. We did find multiple solutions for LECs which describe the data equally well if we neglect isospin breaking and fix $\tilde{C}_{1}^{n p}, C_{S_{1}}, D_{S_{0}}^{1}, D_{S_{1}}^{2}$ from a fit to the Nijmegen $n p$ phase shift. Taking into account isospin breaking effects and performing a combined fit to both $p p$ and $n p$ phase shifts turns out to improve the situation and help to sort out the true solution. We found a single solution for the LECs for the cut-off combinations $\{\Lambda, \tilde{\Lambda}\}=\{450,700\}$ and $\{600,700\}$. For the two other cut-off combinations several local minima in the $\chi^{2}$-plot have been observed. We then adopted the values for the LECs corresponding to the global minimum. We have checked that these values result from the ones for different $\{\Lambda, \tilde{\Lambda}\}$ by a continuous change of the cut-offs.

The situation in the $\left({ }^{3} S_{1}-{ }^{3} D_{1}\right)$-channel is even more complex since one has to determine eight LECs. Our results for the LECs are shown in Tables 2-4. Due to the large dimension of the parameter space, we cannot definitely claim that the found values for the LECs correspond to a true global minimum of the $\chi^{2}$.

Let us now comment on the naturalness of the determined LECs. In general, the natural size for the LECs can be (roughly) estimated as follows:

$$
\tilde{C}_{i} \sim \frac{4 \pi}{F_{\pi}^{2}}, \quad C_{i} \sim \frac{4 \pi}{F_{\pi}^{2} \Lambda_{\mathrm{LEC}}^{2}}, \quad D_{i} \sim \frac{4 \pi}{F_{\pi}^{2} \Lambda_{\mathrm{LEC}}^{4}},
$$

where $\Lambda_{\mathrm{LEC}}$ is the scale entering the values of the LECs and the factor $4 \pi$ results from the angular integration in the partial wave decomposition, see Appendix B for the details. The $S$-wave LECs shown in Table 2 are of the natural size except the LECs $D_{{ }^{1} S_{0}}^{1}$ and $D_{3 S_{1}}^{1}$, 
Table 3

The LECs $C_{i}$ and $D_{i}$ in the $P$-waves and $\epsilon_{1}$ at $\mathrm{N}^{3} \mathrm{LO}$ for the different cut-off combinations $\{\Lambda[\mathrm{MeV}], \tilde{\Lambda}[\mathrm{MeV}]\}$. The values of the $C_{i}\left(D_{i}\right)$ are in $10^{4} \mathrm{GeV}^{-4}\left(10^{4} \mathrm{GeV}^{-6}\right)$

\begin{tabular}{lcccr}
\hline LEC & $\{450,500\}$ & $\{600,600\}$ & $\{450,700\}$ & $\{600,700\}$ \\
\hline$C_{1} P_{1}$ & 0.1862 & 0.3374 & 0.2072 & 0.3444 \\
$D_{1} P_{1}$ & 2.3257 & 1.9180 & 2.3968 & 1.9213 \\
$C_{3} P_{0}$ & 1.1729 & 1.2034 & 1.1913 & 1.2031 \\
$D^{3} P_{0}$ & 1.0892 & 1.2500 & 1.2190 & 1.4116 \\
$C_{3} P_{1}$ & -0.6334 & -0.6602 & -0.7576 & -0.7193 \\
$D_{3} P_{1}$ & 4.2369 & 3.8465 & 4.2099 & 3.8756 \\
$C_{3} P_{2}$ & -0.5542 & -0.5812 & -0.6217 & -0.6114 \\
$D_{3} P_{2}$ & 4.1956 & 4.2270 & 4.0340 & 4.1723 \\
$C_{\epsilon}$ & -0.4516 & -0.2726 & -0.5045 & -0.3352 \\
$D_{\epsilon}$ & 2.6303 & 1.7686 & 2.0296 & 1.5516 \\
\hline
\end{tabular}

Table 4

The $D$-wave LECs $D_{i}$ at $\mathrm{N}^{3} \mathrm{LO}$ for the different cut-off combinations $\{\Lambda[\mathrm{MeV}], \tilde{\Lambda}[\mathrm{MeV}]\}$. The values of the $D_{i}$ are in $10^{4} \mathrm{GeV}^{-6}$

\begin{tabular}{lcccc}
\hline LEC & $\{450,500\}$ & $\{600,600\}$ & $\{450,700\}$ & $\{600,700\}$ \\
\hline$D_{1} D_{2}$ & -2.2450 & -2.1874 & -2.3398 & -2.2203 \\
$D D_{1}$ & -1.3988 & -1.7483 & -1.2250 & -1.6620 \\
$D_{3} D_{2}$ & -1.4180 & -0.9023 & -1.3578 & -0.8580 \\
$D_{3} D_{3}$ & -2.0792 & -1.5493 & -1.7522 & -1.4841 \\
$D_{\epsilon 2}$ & 0.2333 & 0.2901 & 0.2274 & 0.2892 \\
\hline
\end{tabular}

which are somewhat large in magnitude. Indeed, estimating the scale $\Lambda_{\text {LEC }}$ as $\Lambda_{\text {LEC }} \sim$ $500 \mathrm{MeV}$ leads to $\left|D_{i}\right| \sim 2.4$ in the same units as used in Table 2. Still, the higher-order contact interactions are suppressed compared to the lower-order operators at low momenta. For example, for the cut-off combination $\{450,500\}$ and $p=p^{\prime}=M_{\pi}$ the contributions of the contact operators at various orders are given by

$$
\begin{aligned}
& \left.\left\langle{ }^{1} S_{0}\left|V_{\mathrm{cont}}^{n p}\left(p, p^{\prime}\right)\right|{ }^{1} S_{0}\right)\right|_{p=p^{\prime}=M_{\pi}} \\
& \quad=\left[\tilde{C}_{{ }^{1} S_{0}}^{n p}+C_{{ }^{1} S_{0}}\left(p^{2}+p^{\prime 2}\right)+\left(D_{{ }^{1} S_{0}}^{1} p^{2} p^{\prime 2}+D_{{ }^{1} S_{0}}^{2}\left(p^{4}+p^{\prime 4}\right)\right)\right]_{p=p^{\prime}=M_{\pi}} \\
& \quad=[-0.091+0.057+(-0.010+0.003)] \times 10^{4} \mathrm{GeV}^{-2} .
\end{aligned}
$$

\subsubsection{P-waves}

Our results for the $n p P$-waves and the mixing angle $\epsilon_{1}$ are shown in Fig. 4. All phase shifts are visibly improved compared to the NLO and NNLO results. One has, however, to keep in mind that two independent parameters appear now in each of these channels instead of one parameter at both NLO and NNLO. The results of the Nijmegen PWA are reproduced in our $\mathrm{N}^{3} \mathrm{LO}$ analysis within the theoretical uncertainty in all phase shifts with exception of ${ }^{1} P_{1}$ at larger energies. In the case of the ${ }^{3} P_{2}$ partial wave, the band is dominated by variation of the SFR cut-off. In particular, lower values for this cut-off lead to 

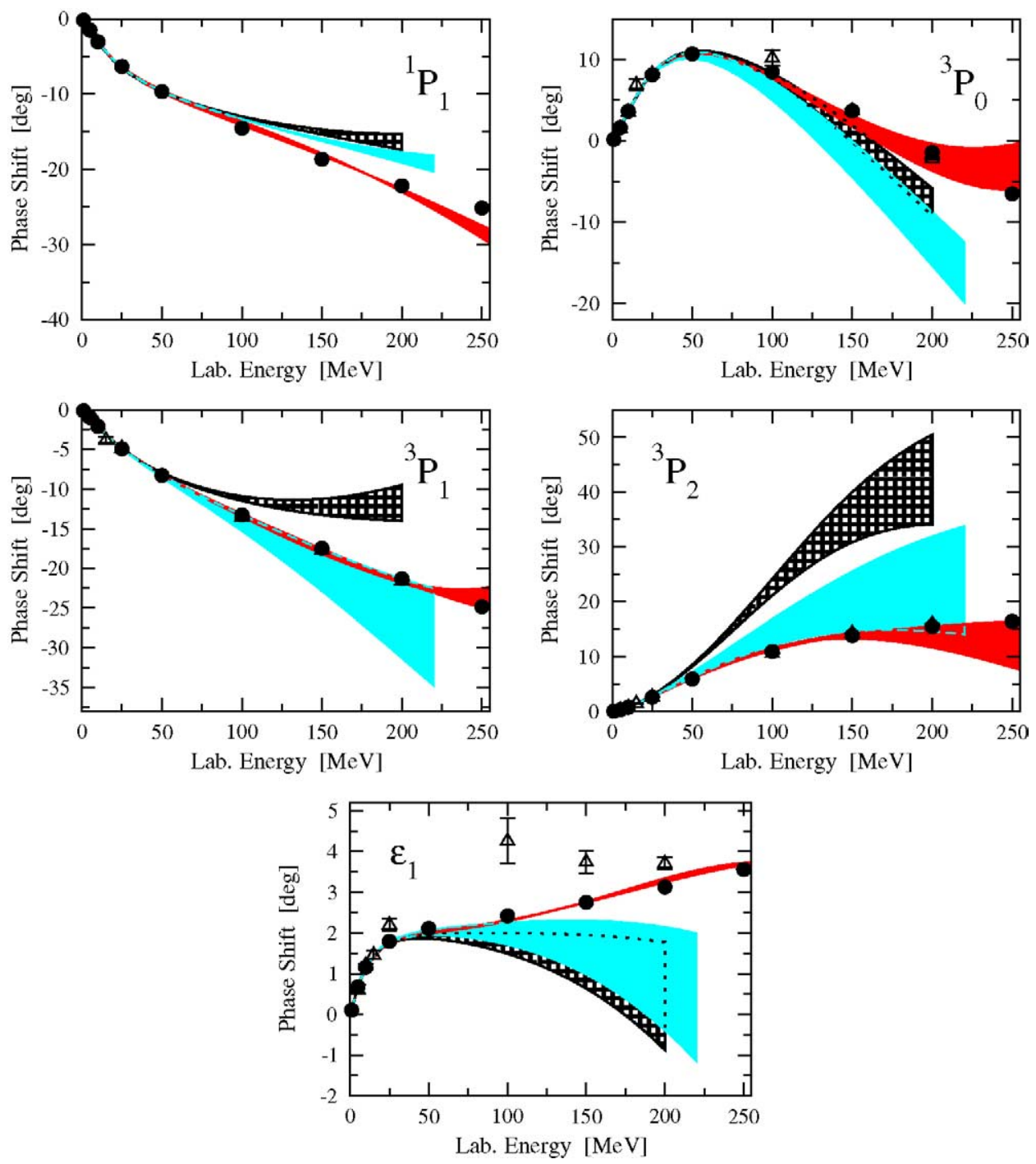

Fig. 4. $P$-wave $n p$ phase shifts and mixing angle $\epsilon_{1}$ versus the nucleon laboratory energy. For notation see Fig. 3.

a better agreement with the data at higher energies. At $E_{\text {lab }}=250 \mathrm{MeV}$, the $\mathrm{N}^{3} \mathrm{LO}$ phase shifts deviate from the data by an amount of up to $\sim 8^{\circ}$. The typical size of the $P$-wave phase shifts at this energy is of the order $\sim 25^{\circ}$. The uncertainty in the calculated phase shifts due to the cut-off variation agrees therefore fairly well with the estimation in Section 6.1. We remind the reader that the theoretical bands at NLO and NNLO are expected to have a similar width, since the effective potential at these orders contains the same set of contact interactions (counter terms). As explained in detail in [21], the uncertainty re- 


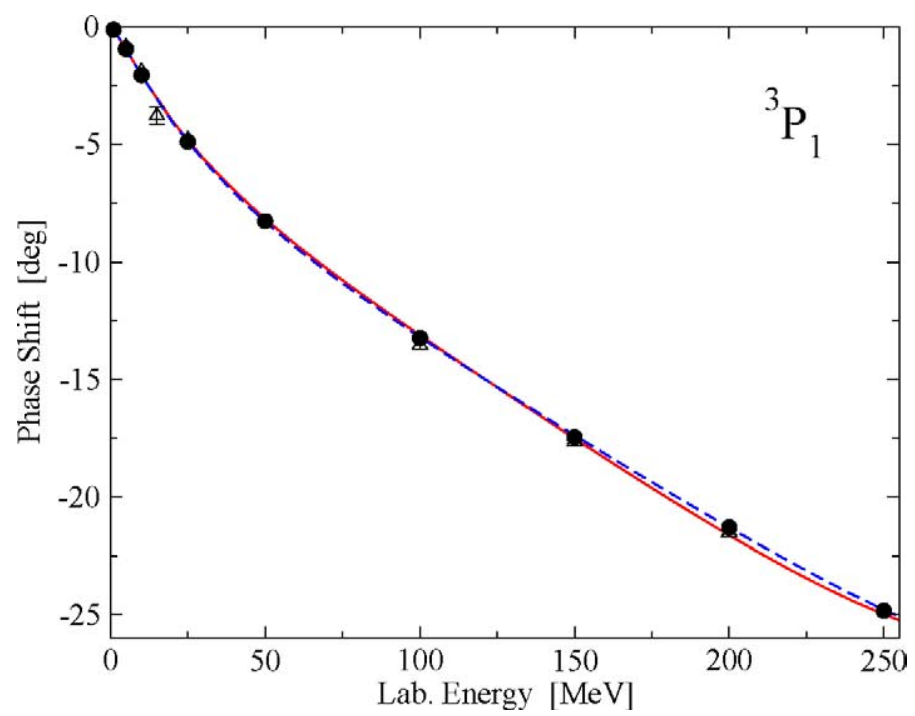

Fig. 5. ${ }^{3} P_{1}$-wave $n p$ phase shift versus the nucleon laboratory energy. The solid and dashed lines correspond to the LECs $C_{3} P_{1}$ and $D_{3} P_{1}$ from the first and second lines in Eq. (6.3), respectively. For remaining notations see Fig. 3.

sulting from the cut-off variation at NLO is smaller than the actual theoretical uncertainty at this order.

The pertinent $P$-wave LECs are tabulated in Table 3 four these four pairs of cut-offs. As in the previously discussed case of the $S$-waves, we found multiple solutions for the LECs. The physical solution can easily be determined due to the smaller number of parameters (two unknown LECs in each $P$-wave). To illustrate this point consider the ${ }^{3} P_{1}$ partial wave with the cut-offs $\{\Lambda, \tilde{\Lambda}\}=\{450,500\}$. We find two solutions for the LECs $C_{3} P_{1}$ and $D^{3} P_{1}$ fitting to the Nijmegen $p p$ phase shifts:

$$
\begin{aligned}
& C_{3 P_{1}}=-0.6334, \quad D^{3} P_{1}=4.2359, \\
& C^{C_{P_{1}}}=5.9620, \quad D^{3} P_{1}=-20.6154,
\end{aligned}
$$

where we used the same units as in Table 3. Both sets of parameters lead to an accurate description of the data, which is shown in Fig. 5. The solution in the first line of Eq. (6.3) satisfies the naturalness assumption for the LECs and has been adopted in the present analysis. Notice further that the value $C_{3} P_{1}=-0.6334 \times 10^{4} \mathrm{GeV}^{-2}$ is close to the NLO and NNLO values for these LECs (for the same cut-off combination) $C_{3} P_{1}=-0.4932 \times$ $10^{4} \mathrm{GeV}^{-2}$ and $C_{3 P_{1}}=-0.7234 \times 10^{4} \mathrm{GeV}^{-2}$, respectively. The results for other partial waves are similar. All LECs $C_{i}$ in the $P$-waves are found to be of natural size and take the values which are close to the ones at NLO and NNLO. The $P$-wave LECs $D_{i}$ are natural as well.

Let us now comment on isospin-breaking. As already explained before, the LECs in the isovector partial waves are fitted to the $p p$ phase shifts. To calculate the corresponding $n p$ phase shifts, we switch off the electromagnetic interaction and adjust for the proper pion 
Table 5

Effects (in degrees) on the phase shifts $\delta_{i}, i=\left\{{ }^{3} P_{0},{ }^{3} P_{1},{ }^{3} P_{2},{ }^{1} D_{2}\right\}$, due to removal of the Coulomb interactions $\left(\Delta_{i}^{\gamma}\right)$ and subsequently including the pion mass differences in the $1 \mathrm{PE}$ potential $\left(\Delta_{i}^{\pi}\right)$ at $E_{\text {lab }}=10 \mathrm{MeV}$. The shifts $\Delta_{i}^{\gamma}, \Delta_{i}^{\pi}$ and $\Delta_{i}$ are defined as follows: $\Delta_{i}^{\gamma}=\left(\bar{\delta}_{p p}\right)_{i}-\left(\delta_{p p}\right)_{i}, \Delta_{i}^{\pi}=\left(\delta_{n p}\right)_{i}-\left(\bar{\delta}_{p p}\right)_{i}, \Delta_{i} \equiv \Delta_{i}^{\gamma}+\Delta_{i}^{\pi}=$ $\left(\delta_{n p}\right)_{i}-\left(\delta_{p p}\right)_{i}$ and $\left(\bar{\delta}_{p p}\right)_{i}$ denotes the $p p$ phase shifts calculated in the absence of the Coulomb interaction. The Nijmegen PWA results are from [38]. The cut-offs $\Lambda$ and $\tilde{\Lambda}$ are varied as specified in Eq. (5.1)

\begin{tabular}{|c|c|c|c|c|c|c|}
\hline \multirow[t]{2}{*}{$i$} & \multicolumn{3}{|l|}{ Chiral $\mathrm{N}^{3} \mathrm{LO}$} & \multicolumn{3}{|c|}{ Nijmegen PWA } \\
\hline & $\overline{\Delta_{i}^{\gamma}}$ & $\Delta_{i}^{\pi}$ & $\Delta_{i}$ & $\overline{\Delta_{i}^{\gamma}}$ & $\Delta_{i}^{\pi}$ & $\Delta_{i}$ \\
\hline$\overline{{ }^{3} P_{0}}$ & $0.359-0.360$ & $(-0.456)-(-0.454)$ & $(-0.096)-(-0.094)$ & 0.371 & -0.447 & -0.076 \\
\hline${ }^{3} P_{1}$ & $(-0.187)-(-0.184)$ & $0.191-0.192$ & $0.005-0.008$ & -0.186 & 0.183 & -0.003 \\
\hline${ }^{3} P_{2}$ & $0.092-0.093$ & $(-0.031)-(-0.030)$ & $0.061-0.062$ & 0.092 & -0.035 & 0.057 \\
\hline${ }^{1} \mathrm{D}_{2}$ & $0.014-0.015$ & $(-0.024)-(-0.024)$ & $(-0.010)-(-0.009)$ & 0.014 & -0.023 & -0.009 \\
\hline
\end{tabular}

Table 6

Effects on the phase shifts $\delta_{i}, i=\left\{{ }^{3} P_{0},{ }^{3} P_{1},{ }^{3} P_{2},{ }^{1} D_{2}\right\}$, due to removal of the Coulomb interactions $\left(\Delta_{i}^{\gamma}\right)$ and subsequently including the pion mass differences in the $1 \mathrm{PE}$ potential $\left(\Delta_{i}^{\pi}\right)$ at $E_{\mathrm{lab}}=25 \mathrm{MeV}$. For notations see Table 5

\begin{tabular}{|c|c|c|c|c|c|c|}
\hline \multirow[t]{2}{*}{$i$} & \multicolumn{3}{|l|}{ Chiral $\mathrm{N}^{3} \mathrm{LO}$} & \multicolumn{3}{|c|}{ Nijmegen PWA } \\
\hline & $\overline{\Delta_{i}^{\gamma}}$ & $\Delta_{i}^{\pi}$ & $\Delta_{i}$ & $\overline{\Delta_{i}^{\gamma}}$ & $\Delta_{i}^{\pi}$ & $\Delta_{i}$ \\
\hline$\overline{{ }^{3} P_{0}}$ & $0.320-0.325$ & $(-0.789)-(-0.784)$ & $(-0.465)-(-0.464)$ & 0.342 & -0.785 & -0.443 \\
\hline${ }^{3} P_{1}$ & $(-0.222)-(-0.218)$ & $0.293-0.293$ & $0.071-0.075$ & -0.221 & 0.275 & 0.054 \\
\hline${ }^{3} P_{2}$ & $0.185-0.190$ & $(-0.099)-(-0.093)$ & $0.088-0.096$ & 0.184 & -0.115 & 0.069 \\
\hline${ }^{1} D_{2}$ & $0.029-0.031$ & $(-0.048)-(-0.048)$ & $(-0.020)-(-0.017)$ & 0.031 & -0.046 & -0.015 \\
\hline
\end{tabular}

Table 7

Effects on the phase shifts $\delta_{i}, i=\left\{{ }^{3} P_{0},{ }^{3} P_{1},{ }^{3} P_{2},{ }^{1} D_{2}\right\}$, due to removal of the Coulomb interactions $\left(\Delta_{i}^{\gamma}\right)$ and subsequently including the pion mass differences in the $1 \mathrm{PE}$ potential $\left(\Delta_{i}^{\pi}\right)$ at $E_{\text {lab }}=50 \mathrm{MeV}$. For notations see Table 5

\begin{tabular}{|c|c|c|c|c|c|c|}
\hline \multirow[t]{2}{*}{$i$} & \multicolumn{3}{|l|}{ Chiral $\mathrm{N}^{3} \mathrm{LO}$} & \multicolumn{3}{|c|}{ Nijmegen PWA } \\
\hline & $\overline{\Delta_{i}^{\gamma}}$ & $\Delta_{i}^{\pi}$ & $\Delta_{i}$ & $\Delta_{i}^{\gamma}$ & $\Delta_{i}^{\pi}$ & $\Delta_{i}$ \\
\hline${ }^{3} P_{0}$ & $0.091-0.109$ & $(-0.879)-(-0.866)$ & $(-0.775)-(-0.770)$ & 0.119 & -0.896 & -0.777 \\
\hline${ }^{3} P_{1}$ & $(-0.236)-(-0.227)$ & $0.320-0.323$ & $0.084-0.095$ & -0.233 & 0.297 & 0.064 \\
\hline${ }^{3} P_{2}$ & $0.251-0.260$ & $(-0.175)-(-0.161)$ & $0.078-0.098$ & 0.253 & -0.221 & 0.032 \\
\hline${ }^{1} D_{2}$ & $0.045-0.050$ & $(-0.041)-(-0.041)$ & $0.003-0.008$ & 0.049 & -0.034 & 0.015 \\
\hline
\end{tabular}

mass in the $1 \mathrm{PE}$ potential. The differences between the corresponding $p p$ and $n p$ phase shifts at three different energies $E_{\text {lab }}=10,25$ and $50 \mathrm{MeV}$ are shown in Tables 5, 6 and 7, respectively. In general, we see that the effects due to removal of the Coulomb interaction, $\Delta_{i}^{\gamma}$, agree very well with the ones of Nijmegen PWA. The uncertainty due to the cut-offs variation becomes larger at higher energies. The effects due to including the pion mass difference, $\Delta_{i}^{\pi}$, show typically somewhat larger deviations from the Nijmegen PWA. This is presumably to a large extent due to a different treatment of the 1PE force: while we use the potential in momentum space with high momenta being cut off, the Nijmegen group performs calculations in coordinate space, and chooses to cut-off the long-range potential 
at $1.4 \mathrm{fm}$. Although both methods certainly lead to the same long-distance asymptotics of the $1 \mathrm{PE}$ potential, they differ significantly in the treatment of its shorter-range part. The largest deviations from $\sim 15 \%$ at $E_{\text {lab }}=10 \mathrm{MeV}$ to $\sim 20 \%-30 \%$ at $E_{\text {lab }}=50 \mathrm{MeV}$ for $\Delta_{i}^{\pi}$ from the Nijmegen PWA are observed in the ${ }^{3} P_{2}$ partial wave. It is comforting to see that both isospin-violating effects (i.e., due to the Coulomb force and the pion mass difference in 1PE) are in most cases of the same size, as it is also expected from power counting arguments, see Section 2.4 for more details. We also note that these two effects have often opposite sign and tend to cancel. For example, one observes at $E_{\text {lab }}=10 \mathrm{MeV}$ [38]: $\Delta^{3} P_{0} \equiv \Delta_{3 P_{0}}^{\gamma}+\Delta^{\pi} P_{0}=0.371^{\circ}-0.447^{\circ}=-0.076^{\circ}$.

\subsubsection{D- and higher partial waves}

The results for $D-, F$ - and $G$-waves are shown in Figs. 6, 7 and 8, respectively. We remind the reader that at $\mathrm{N}^{3} \mathrm{LO}$ there is one adjustable constant $D_{i}$ in each of the $D$-waves, while $F$ - and higher partial waves are parameter-free. As depicted in Fig. 6, the shape of the ${ }^{3} D_{3}$ partial wave is still not properly reproduced at $\mathrm{N}^{3} \mathrm{LO}$, although it is greatly improved compared to NLO and NNLO predictions. This phase shift is, however, rather small as compared to other $D$-wave phase shifts, and thus one expects relatively small effect of this phase shift on the $N N$ scattering observables. We also note that the absolute deviation from the data in this channel is not larger than in the other $D$-waves. Most of the $F$-and $G$-waves are at $\mathrm{N}^{3} \mathrm{LO}$ in agreement with the data. One observes that the theoretical bands do not get thinner at $\mathrm{N}^{3} \mathrm{LO}$, which might at first sight appear strange. This, however, is naturally explained by the fact that there are no short-range contact terms in these channels. Such terms start to contribute to $F$-waves at $\mathrm{N}^{5} \mathrm{LO}\left(Q^{6}\right)$ and to $G$-waves at $\mathrm{N}^{7} \mathrm{LO}$ $\left(Q^{8}\right)$. Consequently, one should expect the uncertainty due to the cut-off variation to be of the same size for calculations up to these high orders in the chiral expansion. Clearly, peripheral partial waves are strongly dominated by the 1PE potential, which represents the longest-range part of the strong nuclear force. Indeed, one observes that the phase shifts are mostly well reproduced already at NLO, while NNLO and $\mathrm{N}^{3} \mathrm{LO}$ corrections only produce minor changes. Notice further that due to the smallness of the phase shifts, the Born approximation works very well in high partial waves and the phase shifts are essentially given by the diagonal (in momentum space) matrix elements of the two-nucleon potential. It is then clear that the bands arise almost completely due to multiplying the potential by the regulator function. The only exception from this rule is given by the ${ }^{1} D_{2}$ partial wave, where the SFR cut-off has a larger impact on the phase shift at higher energy than the cut-off in the Lippmann-Schwinger equation. In particular, the lower values of this cut-off lead to larger values of the phase shift.

The determined LECs $D_{i}$ are tabulated in Table 4. All of them are of natural size.

\subsection{S-wave effective range expansion}

We now regard the $S$-wave effective range parameters and begin with the $n p$ system. In that case one can make use of the usual effective range expansion for finite-range potentials, Eq. (D.1). The reason is that the long-range magnetic moment interaction does not contribute to states with $l=0$. Notice, however, that one should not use the standard effective range expansion for the ${ }^{3} D_{1}$ partial wave and mixing angle $\epsilon_{1}$, which are modified in 

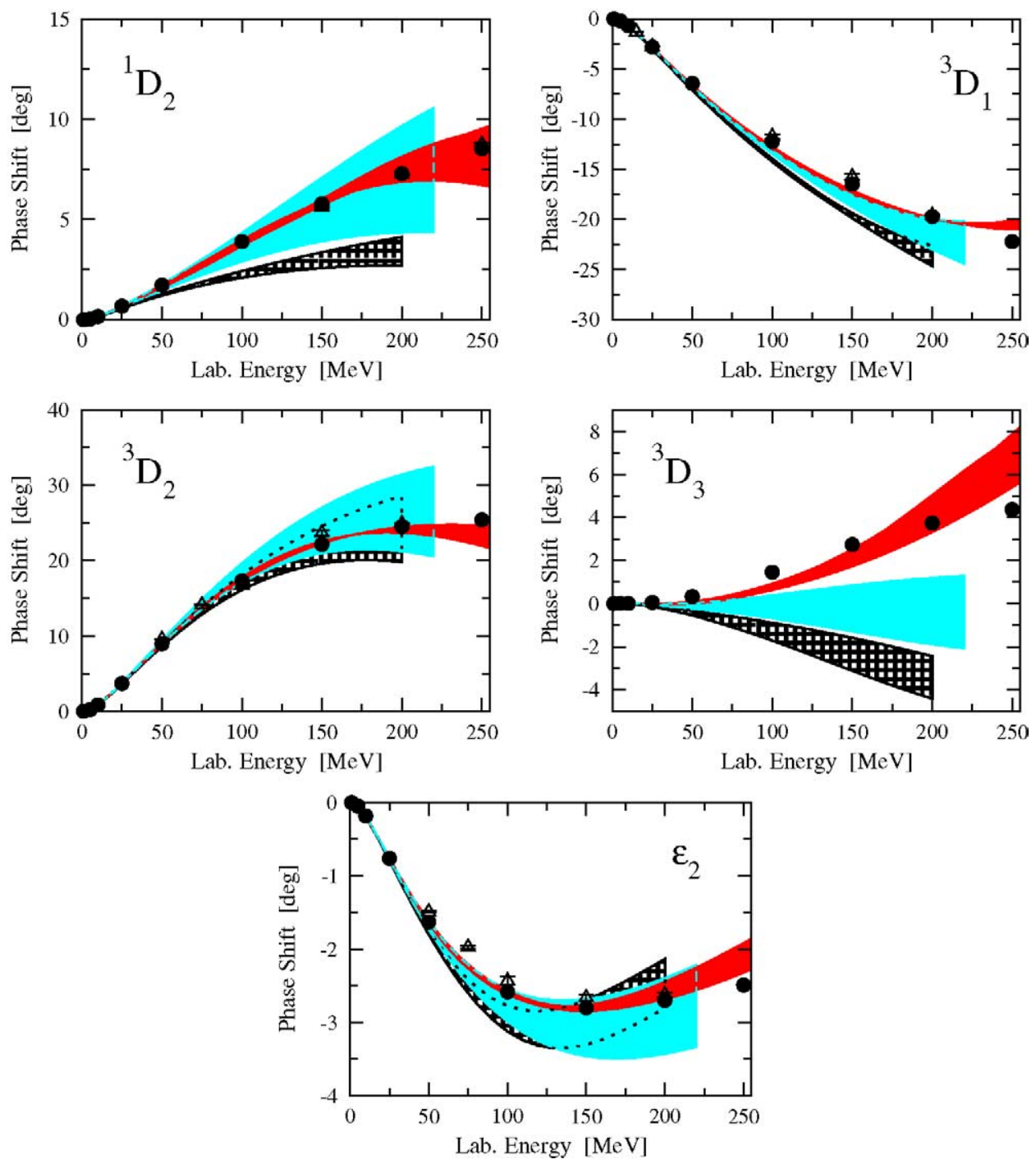

Fig. 6. $D$-wave $n p$ phase shifts and mixing angle $\epsilon_{2}$ versus the nucleon laboratory energy. For notation see Fig. 3.

the presence of the long-range $\left(\sim 1 / r^{3}\right)$ magnetic moment interaction. Our results for the ${ }^{3} S_{1}$ and ${ }^{1} S_{0}$ scattering length, effective range and shape coefficients $v_{2,3,4}$ are summarized in Tables 8 and 9. The results for the ${ }^{1} S_{0}$ scattering length and effective range are improved compared to the NLO and NNLO predictions of [21]. The $\mathrm{N}^{3} \mathrm{LO}$ result for the scattering length fills a small gap between the NNLO prediction and the value of the Nijmegen PWA. The uncertainty due to the cut-off variation for all effective range parameters turns out to be smaller at $\mathrm{N}^{3} \mathrm{LO}$ compared to NNLO, as it should. We observe a minor discrepancy for the shape coefficient $v_{2}$, which might however simply reflect the lack of numerical ac- 

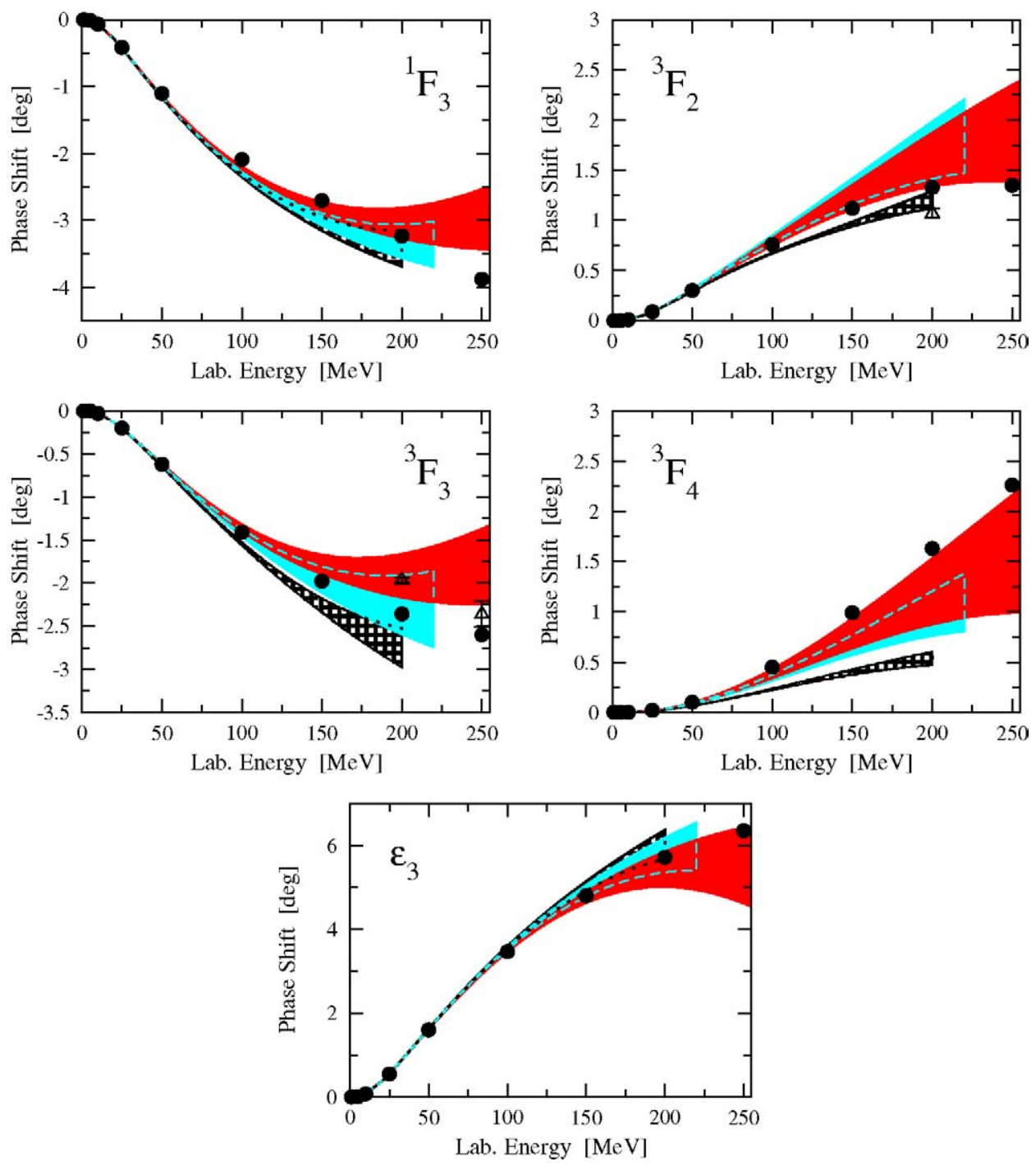

Fig. 7. $F$-wave $n p$ phase shifts and mixing angle $\epsilon_{3}$ versus the nucleon laboratory energy. For notation see Fig. 3.

curacy, with which this quantity is calculated. The description of the ${ }^{3} S_{1}$ effective range parameters is similar to the one in the ${ }^{1} S_{0}$ channel.

Next, we consider the $p p$ system. This case is much more complex since one has to account for electromagnetic interaction. Ideally, one should use the phase shifts $\delta_{\mathrm{EM}+\mathrm{N}}^{\mathrm{EM}}$ and the expression for the effective range function given in [50] to obtain the effective range expansion for the nuclear force in presence of the long-range electromagnetic interactions, which in the ${ }^{1} S_{0}$ channel are given by the improved Coulomb and vacuum polarization potentials in Eqs. (2.53) and (2.56). In the present analysis we have used a simplified de- 

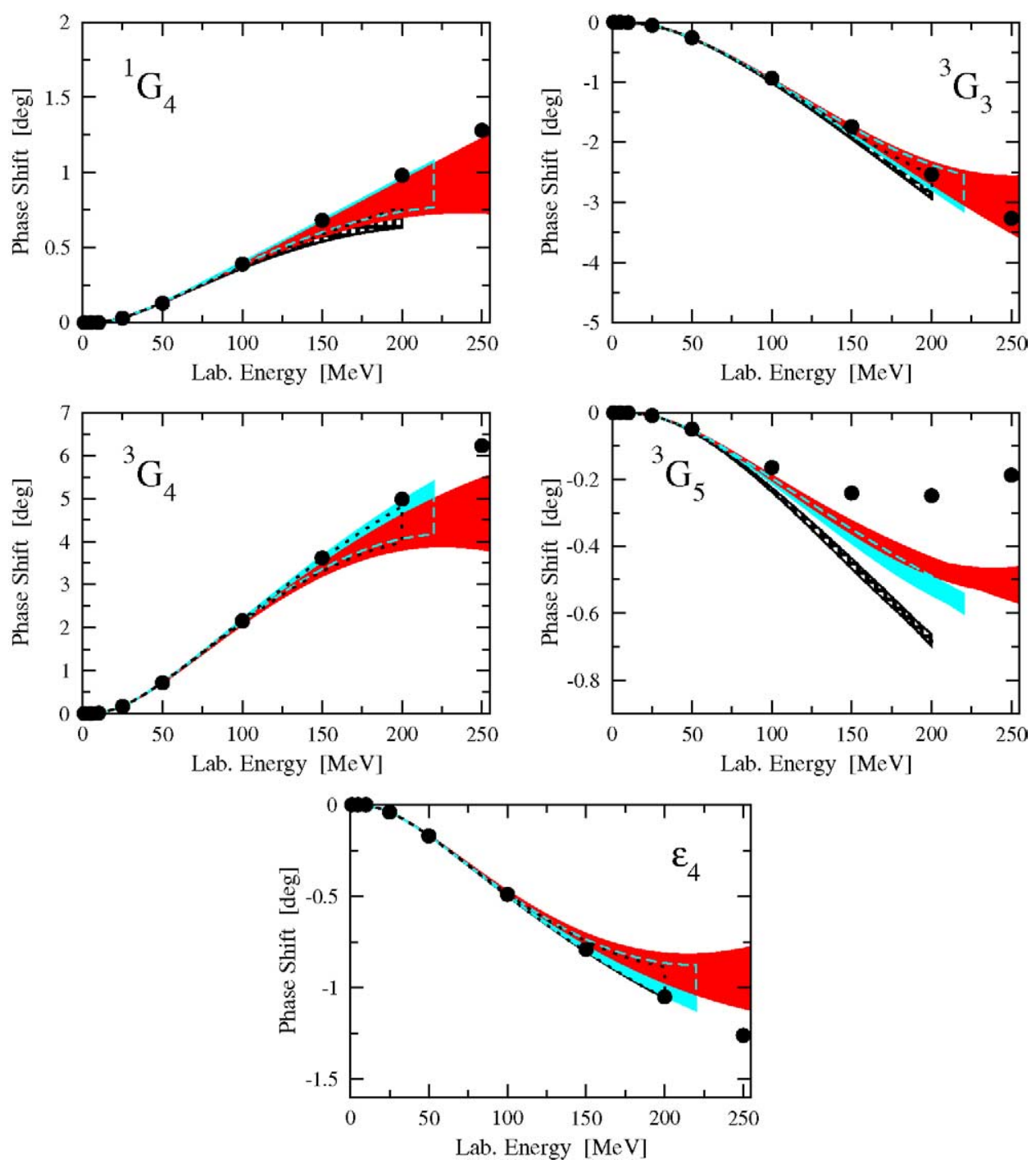

Fig. 8. $G$-wave $n p$ phase shifts and mixing angle $\epsilon_{4}$ versus the nucleon laboratory energy. For notation see Fig. 3.

scription for the $p p$ phase shift as explained in Section 5. We do not calculate explicitly the phase shifts $\delta_{\mathrm{EM}+\mathrm{N}}^{\mathrm{EM}}$ but rather the ones $\delta_{\mathrm{C} 1+\mathrm{N}}^{\mathrm{C} 1}$ of nuclear plus modified Coulomb potential with respect to Coulomb wave functions, adjusting for the difference as explained in Section 5. We have therefore made use of Eq. (D.5) to calculate the $p p$ scattering length and effective range. We obtain the following values:

$$
a_{p p}=(-7.795)-(-7.812) \mathrm{fm}, \quad r_{p p}=2.73-2.76 \mathrm{fm},
$$


Table 8

Scattering length and range parameters for the ${ }^{1} S_{0}$ partial wave using the NLO and NNLO potential [21] compared to the $\mathrm{N}^{3}$ LO results and to the Nijmegen phase shift analysis (PWA). The values $v_{2,3,4}$ are based on the $n p$ Nijm II potential and the values of the scattering length and the effective range are from Ref. [101]

\begin{tabular}{lllcc}
\hline & NLO & NNLO & $\mathrm{N}^{3} \mathrm{LO}$ & Nijmegen PWA \\
\hline$a[\mathrm{fm}]$ & $(-23.447)-(-23.522)$ & $(-23.497)-(-23.689)$ & $(-23.585)-(-23.736)$ & -23.739 \\
$r[\mathrm{fm}]$ & $2.60-2.62$ & $2.62-2.67$ & $2.64-2.68$ & 2.68 \\
$v_{2}\left[\mathrm{fm}^{3}\right]$ & $(-0.46)-(-0.47)$ & $(-0.48)-(-0.52)$ & $(-0.49)-(-0.51)$ & -0.48 \\
$v_{3}\left[\mathrm{fm}^{5}\right]$ & $4.3-4.4$ & $4.0)-4.2$ & $4.0-4.1$ & 4.0 \\
$v_{4}\left[\mathrm{fm}^{7}\right]$ & $(-20.7)-(-21.0)$ & $(-19.9)-(-20.5)$ & $(-19.8)-(-20.2)$ & -20.0 \\
\hline
\end{tabular}

Table 9

Scattering length and range parameters for the ${ }^{3} S_{1}$ partial wave using the CR NLO and NNLO potential [21] compared to the $\mathrm{N}^{3} \mathrm{LO}$ results and to the Nijmegen PWA [93]

\begin{tabular}{lcccc}
\hline & NLO & NNLO & $\mathrm{N}^{3} \mathrm{LO}$ & Nijmegen PWA \\
\hline$a[\mathrm{fm}]$ & $5.429-5.433$ & $5.424-5.427$ & $5.414-5.420$ & 5.420 \\
$r[\mathrm{fm}]$ & $1.710-1.722$ & $1.727-1.735$ & $1.743-1.746$ & 1.753 \\
$v_{2}\left[\mathrm{fm}^{3}\right]$ & $0.06-0.07$ & $0.04-0.05$ & $0.04-0.05$ & 0.04 \\
$v_{3}\left[\mathrm{fm}^{5}\right]$ & $0.77-0.81$ & $0.71-0.76$ & $0.69-0.70$ & 0.67 \\
$v_{4}\left[\mathrm{fm}^{7}\right]$ & $(-4.3)-(-4.4)$ & $(-4.1)-(-4.2)$ & $(-4.0)-(-4.1)$ & -4.0 \\
\hline
\end{tabular}

where the uncertainty is due to the cut-off variation. These values agree nicely with the experimental ones [82]:

$$
a_{p p}^{\exp }=-7.8149 \pm 0.0029 \mathrm{fm}, \quad r_{p p}^{\exp }=2.769 \pm 0.014 \mathrm{fm} .
$$

One should, however, keep in mind that we made an approximation and neglected the effects due to the long-range part of the vacuum polarization potential and the interaction in the second line of Eq. (2.53). As found in [50] neglecting these electromagnetic interactions affects the values of $a_{p p}$ and $r_{p p}$ by an amount smaller than $0.01 \mathrm{fm}$, which is within the theoretical uncertainty of the present analysis.

Finally, we consider the $n n$ system. Since no long-range electromagnetic interactions contribute to the ${ }^{1} S_{0}$ partial wave, one can use the effective range expansion (D.1). Since we have used the "standard value" for the $n n$ scattering length $a_{n n}$

$$
a_{n n}^{\text {std }}=-18.9 \pm 0.4 \mathrm{fm}
$$

as an input to fix the LEC of the leading isospin-violating short-range interaction, we can only make predictions for the effective range $r_{n n}$ :

$$
r_{n n}=2.76-2.80 \mathrm{fm} \text {. }
$$

This agrees with the experimental number [86]

$$
r_{n n}^{\exp }=2.75 \pm 0.11 \mathrm{fm} \text {. }
$$

Notice that there is still some controversy about the experimental value of the $n n$ scattering length extracted using different reactions. For example, the value $a_{n n}^{\exp }=-18.50 \pm 0.53 \mathrm{fm}$ has been reported from studying the ${ }^{2} \mathrm{H}\left(\pi^{-}, n \gamma\right) n$ process [74], while measurements of the 
neutron deuteron breakup reaction lead on one hand to $a_{n n}^{\exp }=-18.7 \pm 0.6 \mathrm{fm}$ [87] and on the other hand to different values, $a_{n n}^{\exp }=-16.1 \pm 0.4 \mathrm{fm}$ and $a_{n n}^{\exp }=-16.3 \pm 0.4 \mathrm{fm}[88]$.

Last but not least, we would like to point out that the relation between the values of the effective range parameters, which would result if there would be no electromagnetic interaction, and the observed ones is highly nontrivial. Neglecting electromagnetic nucleon mass shifts, we can, for example, switch off the Coulomb interaction in the $p p$ system and recalculate the effective range coefficients using Eq. (D.1). This leads to

$$
\tilde{a}_{p p}=(-16.00)-(-16.63) \mathrm{fm}, \quad r_{p p}=2.81-2.86 \mathrm{fm} .
$$

Although the value for $\tilde{a}_{p p}$ is fairly close to the one for the $n n$ and $n p$ scattering lengths, as one would expect from the approximate isospin invariance of the strong interaction, it should be understood that effects due to electromagnetic interaction are not completely removed from these quantities. To clarify this point let us take a look at the leading $n n, n p$ and $p p$ short-range interactions:

$$
\begin{aligned}
& \tilde{C}_{1 S_{0}}^{n n}=\left(\tilde{C}_{1 S_{0}}^{n n}\right)_{\mathrm{str}}+\beta_{n n} \frac{e^{2}}{(4 \pi)^{2}}, \\
& \tilde{C}_{1 S_{0}}^{n p}=\left(\tilde{C}_{1 S_{0}}^{n p}\right)_{\mathrm{str}}+\beta_{n p} \frac{e^{2}}{(4 \pi)^{2}}, \\
& \tilde{C}_{1 S_{0}}^{p p}=\left(\tilde{C}_{1}^{p p}\right)_{\mathrm{str}}+\beta_{p p} \frac{e^{2}}{(4 \pi)^{2}} .
\end{aligned}
$$

Here the LECs $\left(\tilde{C}_{{ }_{1} S_{0}}^{i}\right)_{\text {str }}$ are entirely due to the strong interaction. If only linear terms in the quark mass difference are included, see Eq. (2.35), these LECs are related with each other as $\left(\tilde{C}^{p p}{ }_{S_{0}}\right)_{\mathrm{str}}+\left(\tilde{C}_{{ }^{1} S_{0}}^{n n}\right)_{\mathrm{str}}=2\left(\tilde{C}_{1}^{n p} S_{0}\right)_{\mathrm{str}}$ and the difference $\left(\tilde{C}_{{ }^{1} S_{0}}^{p p}\right)_{\text {str }}-\left(\tilde{C}_{{ }^{1} S_{0}}^{n n}\right)_{\text {str }}$ is proportional to $\epsilon M_{\pi}^{2}$. The terms $\propto \beta_{i}$ in Eq. (6.10) are due to the short-range electromagnetic interactions, see Eq. (2.37). ${ }^{13}$ Since we do not know the values of the LECs $\beta_{i}$ in Eq. (6.10) and it is not possible to disentangle them from $\tilde{C}_{{ }^{1}}^{i}$ in the two-nucleon system, we cannot extract the values for $\mathrm{NN}$ observables due to the strong interaction out of the experimentally measured quantities. Notice that the LECs $\beta_{i}$ might (at least in principle) be determined from processes with external pions. Notice further that the $p p$ scattering length with the long-range Coulomb interaction being switched off is even not a well-defined quantity in an effective field theory approach since it is sensitive to details of the strong interaction at short distances. Indeed, the extracted scattering length $\tilde{a}_{p p}$ in Eq. (6.9) shows a significant cut-off dependence. Clearly, the scattering length due to pure strong interaction is perfectly well defined and the cut-off dependence is (largely) absorbed by the appropriate "running" of $\beta_{p p}$. For related discussion on the proton-proton scattering length in context of effective field theory see $[89,90]$. Furthermore, a useful approximation for the quantity $\tilde{a}_{p p}$ based

\footnotetext{
$\overline{13}$ The fact that we have three independent LECs $\beta_{n n}, \beta_{n p}$ and $\beta_{p p}$ and only two terms in Eq. (2.37) might appear confusing. In fact, we have only shown explicitly electromagnetic isospin-breaking and omitted isospin conserving terms in Eq. (2.37). One of the two electromagnetic isospin conserving contact interactions contribute to ${ }^{1} S_{0}$ and one to ${ }^{3} S_{1} N N$ scattering. Therefore, three and not two independent electromagnetic terms contribute to ${ }^{1} S_{0} N N$ scattering.
} 

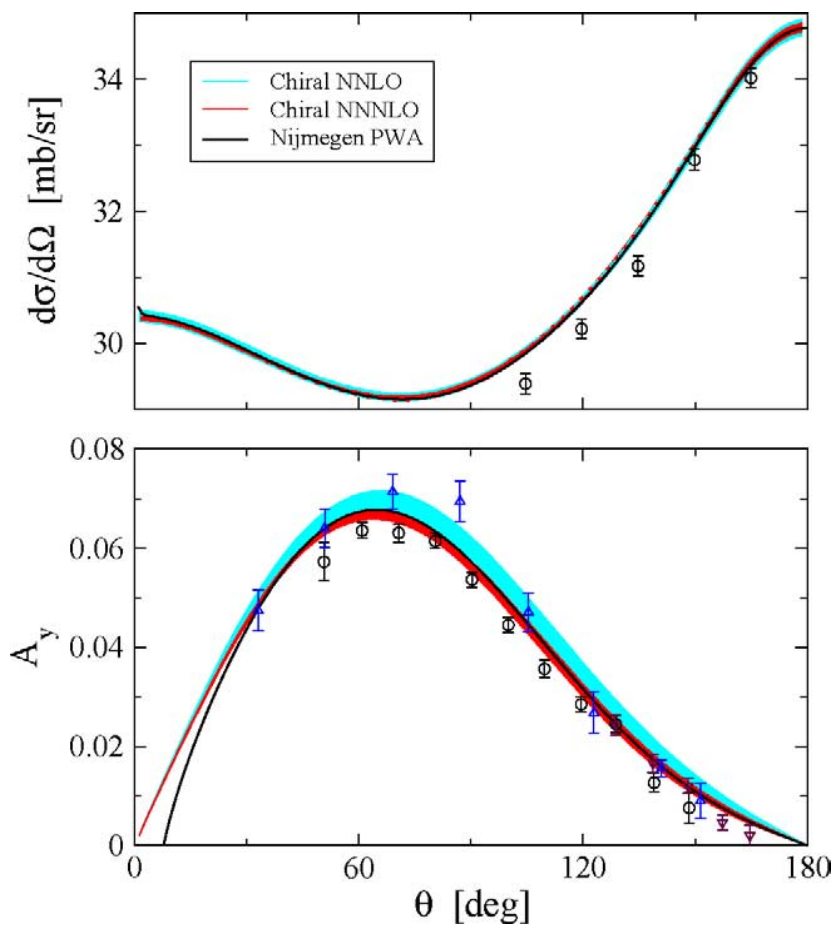

Fig. 9. $n p$ differential cross section and vector analyzing power at $E_{\text {lab }}=25 \mathrm{MeV}$. The Nijmegen PWA result is taken from [107]. Data for the cross section are taken from [108] and for the analyzing power from [109,110]. The cut-offs $\Lambda$ and $\tilde{\Lambda}$ are varied as specified in Eq. (5.1).

on the short-range nature of the strong interaction and the fact that the scattering length is large can be found in [91].

\subsection{Two-nucleon scattering observables}

Once the $N N$ phase shifts are calculated, all two-nucleon scattering observables can be obtained in a straightforward way using, e.g., the formulae collected in [52]. In Figs. 9-12 we show the $n p$ differential cross section and vector analyzing power at $E_{\text {lab }}=25,50,96$ and $143 \mathrm{MeV}$ at NNLO and $\mathrm{N}^{3} \mathrm{LO}$ in comparison with the data and the Nijmegen PWA results. In this calculation, we have included all $n p$ partial waves up to $j \leqslant 8$ and did not take into account the magnetic moment interaction. At the lowest energy we have considered, $E_{\text {lab }}=25 \mathrm{MeV}$, both NNLO and $\mathrm{N}^{3} \mathrm{LO}$ results are consistent with the ones of the Nijmegen PWA. The small disagreement with the Nijmegen PWA in the analyzing power at forward direction is due to the neglected magnetic moment interaction. At higher energies the NNLO predictions become less precise. At $\mathrm{N}^{3} \mathrm{LO}$ the uncertainty in the cross-section due to the cut-off variation at the largest energy we have calculated, $E_{\text {lab }}=143 \mathrm{MeV}$, is less than $10 \%$. It is comforting to see that NNLO and $\mathrm{N}^{3} \mathrm{LO}$ results overlap in most cases and are both in agreement with the Nijmegen PWA. We further notice that the small but visible deviations of our $\mathrm{N}^{3} \mathrm{LO}$ result for the differential cross section from the Nijmegen 

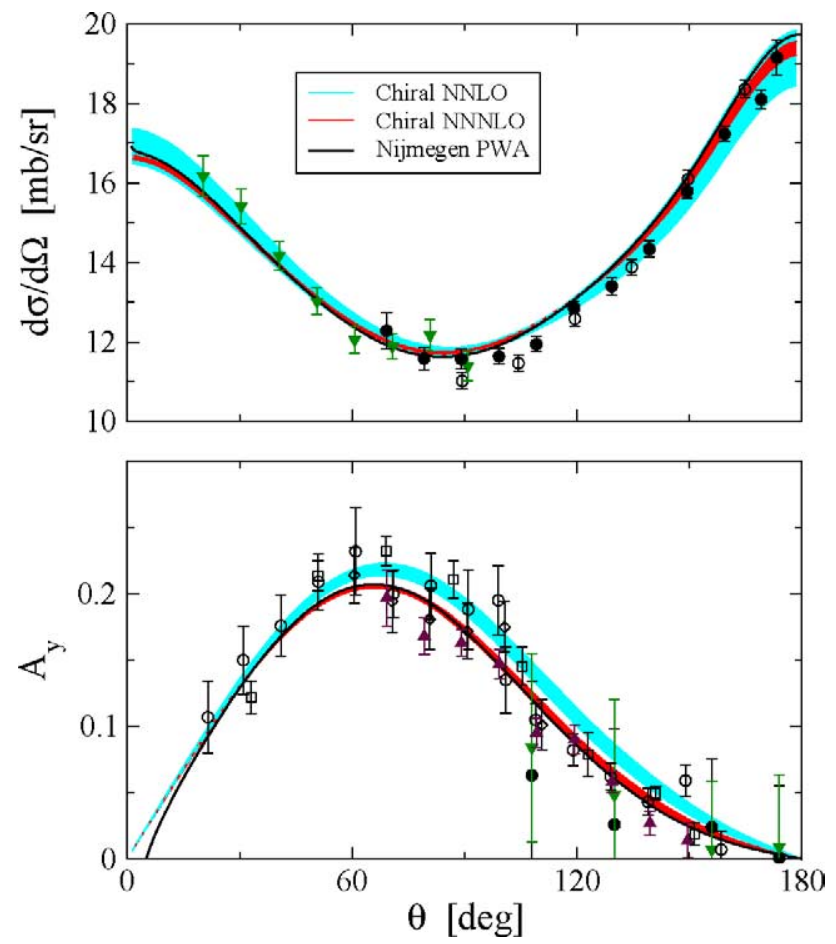

Fig. 10. $n p$ differential cross section and vector analyzing power at $E_{\text {lab }}=50 \mathrm{MeV}$. Data for the cross section are taken from $[108,111]$ and for the analyzing power from [110,112-115]. For remaining notations see Fig. 9.

PWA curve at forward and backward angles and higher energies is most probably due to the lack of partial waves with $j>8$ in our calculations. The convergence of the partial wave expansion is well known to be slow in these particular cases. For example, it has been found in [92] that a sum up to $j=16$ is needed to obtain convergence for the cross section at $E_{\text {lab }}=300 \mathrm{MeV}$ within $1 \%$.

\subsection{Deuteron properties}

We now turn to the bound state properties. We stress that we do not use the deuteron binding energy as a fit parameter as it is frequently done but rather adopt the same LECs as obtained in the fit to the low phases. In Table 10 we collect the resulting deuteron properties in comparison to the NLO and NNLO results from [21]. All results for the deuteron properties in this table have been calculated using the formulae given in section 4 based on the relativistic wave function $\Psi^{\mathrm{d}}(p)$. First, we note a clear improvement at $\mathrm{N}^{3} \mathrm{LO}$ in the chiral expansion. The predicted binding energy at $\mathrm{N}^{3} \mathrm{LO}$ is within $0.4 \%$ of the experimental value. This has to be compared with 1\%-1.5\% ( 2\%-2.5\%) deviation at NNLO (NLO). Also visibly improved is the asymptotic $S$-wave normalization strength $A_{S}$, which now deviates from the experimental (central) value by $0.3 \%$ as compared to $\sim 1.1 \%(\sim 1.9 \%$ ) at NNLO (NLO). Our predictions for the asymptotic $D / S$-ratio have a tendency to slightly 

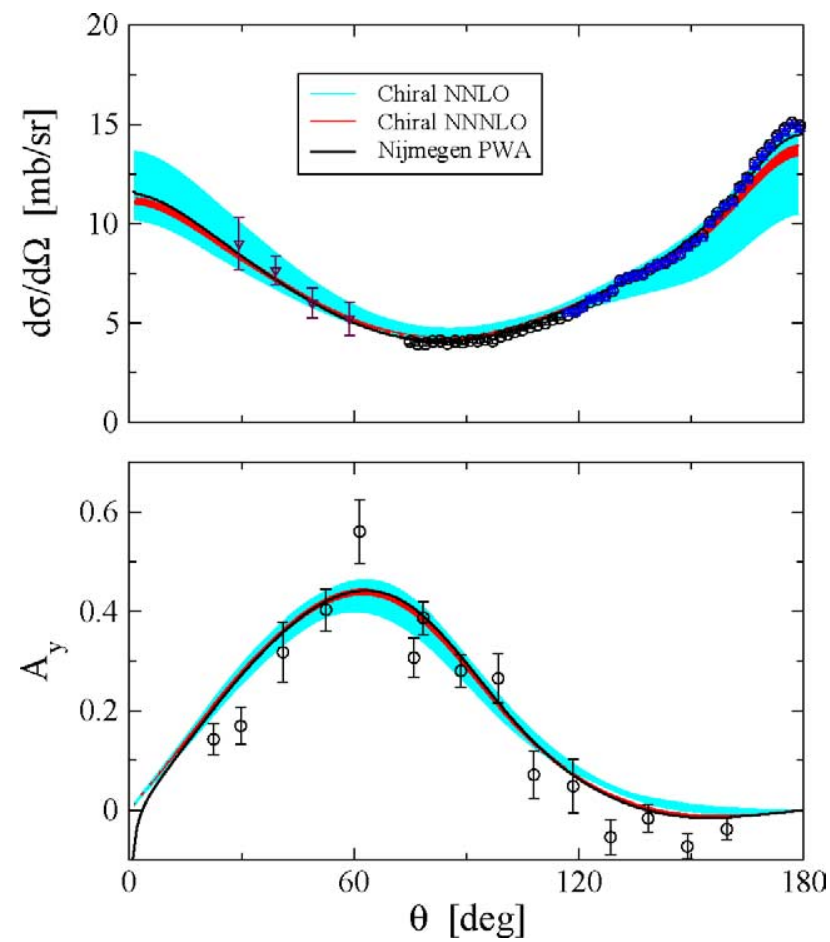

Fig. 11. $n p$ differential cross section and vector analyzing power at $E_{\text {lab }}=96 \mathrm{MeV}$. Data for the cross section are taken from [116,118,119]. Data for the analyzing power are at $E_{\text {lab }}=95 \mathrm{MeV}$ and taken from [117]. For remaining notations see Fig. 9.

Table 10

Deuteron properties derived from the chiral potential at $\mathrm{N}^{3} \mathrm{LO}$ compared to the NLO and NNLO results from [21] and the data. Here, $E_{\mathrm{d}}$ is the binding energy, $Q_{\mathrm{d}}$ the quadrupole moment, $\eta_{\mathrm{d}}$ the asymptotic $D / S$ ratio, $\sqrt{\left\langle r^{2}\right\rangle_{m}^{\mathrm{d}}}$ the root-mean-square matter radius, $A_{S}$ the strength of the asymptotic $S$-wave normalization and $P_{\mathrm{d}}$ the $D$-state probability. The data for $E_{\mathrm{d}}$ are from [102], for $Q_{\mathrm{d}}$ from [103,104], for $\eta_{\mathrm{d}}$ from [105] and for $A_{S}$ from [104]. For the rms-radius we actually show the experimental value for the deuteron "point-nucleon" rms-radius from [64]. In the $\mathrm{N}^{3} \mathrm{LO}$ calculation, the cut-offs are varied as specified in Eq. (5.1)

\begin{tabular}{lllll}
\hline & NLO & NNLO & $\mathrm{N}^{3} \mathrm{LO}$ & Exp \\
\hline$E_{\mathrm{d}}[\mathrm{MeV}]$ & $(-2.171)-(-2.186)$ & $(-2.189)-(-2.202)$ & $(-2.216)-(-2.223)$ & $-2.224575(9)$ \\
$Q_{\mathrm{d}}\left[\mathrm{fm}^{2}\right]$ & $0.273-0.275$ & $0.271-0.275$ & $0.264-0.268$ & $0.2859(3)$ \\
$\eta_{\mathrm{d}}$ & $0.0256-0.0257$ & $0.0255-0.0256$ & $0.0254-0.0255$ & $0.0256(4)$ \\
$\sqrt{\left\langle r^{2}\right\rangle_{m}^{\mathrm{d}}}[\mathrm{fm}]$ & $1.973-1.974$ & $1.970-1.972$ & $1.973-1.985$ & $1.9753(11)$ \\
$A_{S}\left[\mathrm{fm}^{-1 / 2}\right]$ & $0.868-0.873$ & $0.874-0.879$ & $0.882-0.883$ & $0.8846(9)$ \\
$P_{\mathrm{d}}[\%]$ & $3.46-4.29$ & $3.53-4.93$ & $2.73-3.63$ & - \\
\hline
\end{tabular}

reduce its value when going from NLO to NNLO to $\mathrm{N}^{3} \mathrm{LO}$. The results at all orders are in agreement with the data within the experimental uncertainty. Further, our $\mathrm{N}^{3} \mathrm{LO}$ result for $\eta_{\mathrm{d}}$ agrees well with the one of the Nijmegen PWA [93], $\eta_{\mathrm{d}}=0.0253(2)$. We do not ob- 

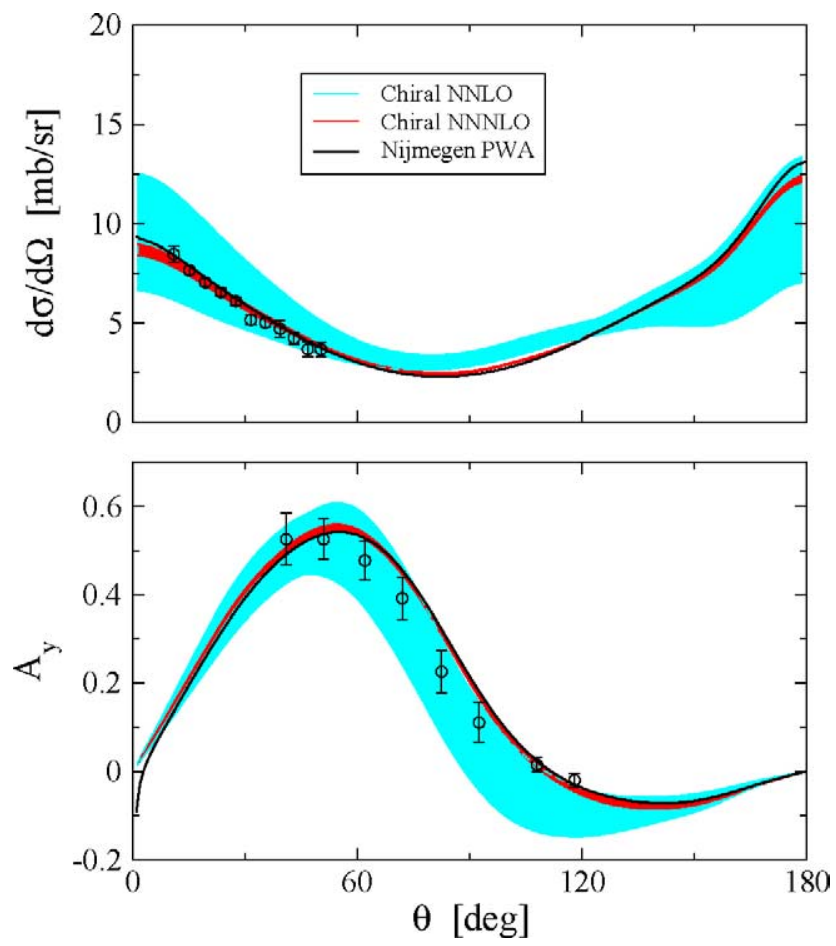

Fig. 12. $n p$ differential cross section and vector analyzing power at $E_{\text {lab }}=143 \mathrm{MeV}$. Data for the cross section are at $E_{\text {lab }}=142.8 \mathrm{MeV}$ and taken from [120] and for the analyzing power from [121]. For remaining notations see Fig. 9.

serve any improvement for the quadrupole momentum $Q_{\mathrm{d}}$ at $\mathrm{N}^{3} \mathrm{LO}$, which shows an even larger deviation from the data compared to NNLO (6\%-8\% versus $4 \%-5 \%)$. We, however, remind the reader that the present calculation of $Q_{\mathrm{d}}$ is based on formulae of Section 4 . It is incomplete and does, in particular, not take into account the contribution of the two-nucleon current. Notice that apart from the pion-exchange two-nucleon currents, there are contributions from two-nucleon contact current, where the corresponding LEC cannot be fixed from nucleon-nucleon scattering. Such current results from the operator in the effective Lagrangian with four nucleon fields, one photon field and two derivatives. It appears natural to fix the value of the accompanying LEC from the requirement to reproduce the value of the deuteron quadrupole moment. For the calculations of the various deuteron properties including the quadrupole moment as well as other two-nucleon observables in pionless EFT the reader might consult Refs. [94,95]. Notice that the situation with the quadrupole moment is analogous to the one described in [96] for the deuteron magnetic moment. In that case the corresponding short-range two-nucleon current results from the operator with just one derivative and thus appears even at a lower order. The situation with the deuteron rms-radius is similar to the one with the quadrupole moment: we observe a larger deviation from the data at $\mathrm{N}^{3} \mathrm{LO}$ as compared to the NLO and NNLO results. Notice however that the deviations at $\mathrm{N}^{3} \mathrm{LO}$ from the experimental number are still of the order of $0.5 \%$ or less. The 
above comment on the missing contributions in the quadrupole moment calculation applies to the deuteron rms-radius as well. At $\mathrm{N}^{3} \mathrm{LO}$ one should account for the contribution due to the short-range two-nucleon current, which results from the contact operator with four nucleon fields, one photon field and two derivatives, see [94] for more details. It is remarkable that the cut-off dependence of $\sqrt{\left\langle r^{2}\right\rangle_{m}^{\mathrm{d}}}$ at $\mathrm{N}^{3} \mathrm{LO}$ is significantly larger compared to NLO and NNLO. This implicitly confirms our previous statement about the necessity to incorporate the short-range current at this order. The cut-off dependence of the corresponding LEC will compensate the cut-off dependence of $\sqrt{\left\langle r^{2}\right\rangle_{m}^{\mathrm{d}}}$ making the deuteron "point-nucleon" radius cut-off independent up to higher-order corrections. The complete $\mathrm{N}^{3} \mathrm{LO}$ calculation of the quadrupole moment and the "point-nucleon" electric charge radius of the deuteron will be presented in a separate publication. As a numerical check, we have recalculated all deuteron properties using the nonrelativistic wave function $\phi^{\mathrm{d}}(p)$ in Eq. (4.3). As expected from the discussion in Section 4, we reproduce the values for $E_{\mathrm{d}}$ and $\eta_{\mathrm{d}}$. The asymptotic $S$-wave normalization $A_{S}$ changes by $\tilde{A}_{S}-A_{S}=0.00039 \mathrm{fm}^{-1 / 2}$. This has to be compared with the value $\tilde{A}_{S}-A_{S}=0.000392 \mathrm{fm}^{-1 / 2}$ from Eq. (4.21). The quadrupole moment and the rms-radius change by $0.4 \%$ and $0.1 \%$, respectively. Finally, we show the deuteron wave function in coordinate space in Fig. 13 for a particular cut-off choice, together with results obtained at NLO and NNLO. One observes a stronger suppression of the $S$-wave component at short distances compared to NLO and NNLO, as well
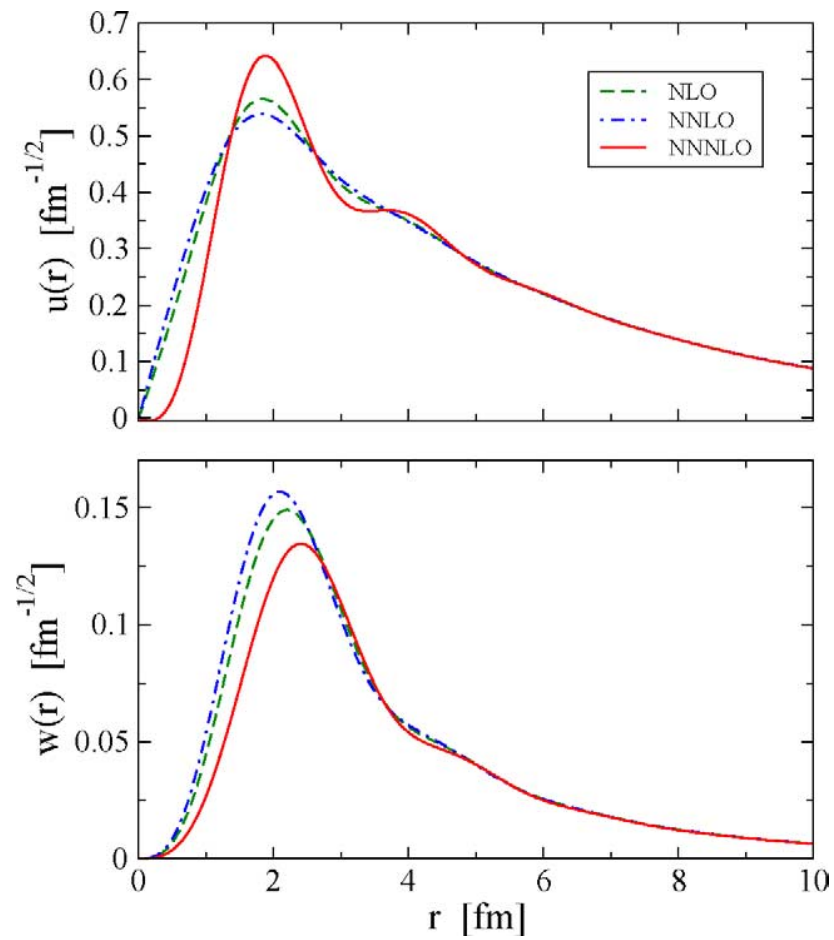

Fig. 13. Coordinate space representation of the $S$ - (upper panel) and $D$-wave (lower panel) deuteron wave functions at NLO, NNLO and $\mathrm{N}^{3} \mathrm{LO}$ for the cut-offs: $\Lambda=550 \mathrm{MeV}, \tilde{\Lambda}=600 \mathrm{MeV}$. 
as the lower probability for the deuteron to be in the $D$-state. The latter observation follows also from the smaller value of $P_{\mathrm{d}}$ quoted in Table 10. The shape of the wave function changes for different cut-off choices. We remind the reader that the deuteron wave function is not observable (except at very large distances).

\section{Summary}

In this paper, we have considered the interactions between two nucleons at $\mathrm{N}^{3} \mathrm{LO}$ in chiral effective field theory. The pertinent results of this study can be summarized as follows:

(i) The two-nucleon potential at $\mathrm{N}^{3} \mathrm{LO}$ consists of one-, two- and three-pion exchanges and a set of contact interactions with zero, two and four derivatives, respectively, according to the chiral power counting, see also Table 1. We have applied spectral function regularization to the multi-pion exchange contributions. This allows for a better separation of the low and high momentum components in the pion loop diagrams than dimensional regularization. Within this framework, we have shown that three-pion exchange can safely be neglected. The corresponding cut-off is varied from 500 to $700 \mathrm{MeV}$. The LECs related to the dimension two and three $\bar{N} N \pi \pi$ vertices are taken consistently from studies of pionnucleon scattering in chiral perturbation theory, [23,73]. In the isospin limit, there are 24 LECs related to four-nucleon interactions which feed into the $S$-, $P$ - and $D$-waves and various mixing parameters, cf. Eq. (2.5).

(ii) We have reviewed the various isospin breaking mechanisms and proposed a novel ordering scheme, based on one small parameter that collects strong as well as electromagnetic isospin violation, cf. Eq. (2.32) accompanied by a particular counting rule for photon loops, see Eq. (2.33). This differs from the scheme proposed and applied in Ref. [7]. In the actual calculations, we have included the leading charge-independence and charge-symmetry breaking four-nucleon operators, the pion mass difference in the $1 \mathrm{PE}$, the kinematical effects due to the nucleon mass difference and the same electromagnetic corrections as done by the Nijmegen group (the static Coulomb potential and various corrections to it, magnetic moment interactions and vacuum polarization). This is done because we fit to the Nijmegen partial waves. In the future, it would be important to also include isospin violation in the $2 \mathrm{PE}, \pi \gamma$-exchange and the isospin breaking corrections to the pion-nucleon scattering amplitude (which have been consistently determined in [44]).

(iii) We have discussed in some detail the form of the scattering equation that is used to iterate the potential and similar for the bound state. We use the Lippmann-Schwinger equation with the relativistic form of the kinetic energy. Such an approach can easily be extended to external probes or few-nucleon systems. We have also discussed the reduction to a nonrelativistic form which be might of easier use in some applications. The LS equation is regulated in the standard way, cf. Eq. (2.28), with the cut-off varied from 450 to $600 \mathrm{MeV}$.

(iv) The total of 26 four-nucleon LECs has been determined by a combined fit to some $n p$ and $p p$ phase shifts from the Nijmegen analysis together with the $n n$ scattering length value $a_{n n}=-18.9 \mathrm{fm}$, as detailed in Section 5. The resulting LECs are of natural size 
except $D_{{ }^{1} S_{0}}^{1}$ and $D_{3}^{1} S_{1}$. Comparing to the fits at NLO and NNLO, we had to extend the fit range to higher energies for the reasons discussed in Section 5.

(v) The description of the low phase shifts $(S, P, D)$ is excellent, see Figs. 3-6. In all cases, the $\mathrm{N}^{3} \mathrm{LO}$ result is better than the NNLO one with a sizeably reduced theoretical uncertainty. This holds in particular for the problematic ${ }^{3} P_{0}$ wave which was not well reproduced at NNLO. The peripheral waves $(F, G, H, \ldots)$, that are free of parameters, are also well described with the expected theoretical uncertainty related to the cut-off variations, see Figs. 7, 8. We stress that the description of the phases in general improves when going from $\mathrm{LO}$ to $\mathrm{NLO}$ to NNLO to $\mathrm{N}^{3} \mathrm{LO}$, as it is expected in a converging EFT.

(vi) The resulting $S$-wave scattering lengths and range parameters in the $n p$ (cf. Tables 8 and 9) and pp systems (cf. Eq. (6.4)) are in good agreement with the ones obtained in the Nijmegen PWA. In addition, we can give theoretical uncertainties for all these quantities, which are mostly in the one percent range.

(vii) The scattering observables (differential cross sections, analyzing powers) for the $n p$ system displayed in Figs. 9-12 are well described, with a small theoretical uncertainty at the order considered here.

(viii) The deuteron properties are further predictions. In particular, we have not included the binding energy in the fits, the deviation from the experimental value is in the range from 0.4 to $0.07 \%$. The asymptotic $S$-wave normalization and the asymptotic $D / S$ are also well described. The remaining discrepancies in the quadrupole moment and the rms matter radius are related to the short-ranged two-nucleon current not considered here.

In the future, these studies should be extended in various directions. In particular, one should construct the electroweak current operators to the same accuracy and work out the corresponding three-nucleon force, which is of special interest since it does not contain any novel LECs. Furthermore, a more systematic study of isospin violation in the two- and three-nucleon systems based on the formalism developed here should be pursued. Work along these lines is under way.

\section{Acknowledgements}

We are grateful to Franz Gross, Barry Holstein, Rocco Schiavilla and Bob Wiringa for several discussions and comments. E.E. also acknowledge the hospitality of the Institute for Nuclear Theory at the University of Washington, Seattle, where part of this research was conducted. This work has been supported by the U.S. Department of Energy Contract No. DE-AC05-84ER40150 under which the Southeastern Universities Research Association (SURA) operates the Thomas Jefferson National Accelerator Facility.

\section{Appendix A. Kinematics}

Consider two nucleons moving with momenta $\vec{p}_{1}$ and $\vec{p}_{2}$. We use the relativistic kinematics for relating the energy $E_{\text {lab }}$ of two nucleons in the laboratory system to the square 
of the nucleon momentum $\vec{p}$ in the center-of-mass system, which is defined by the condition $\vec{p}_{1}+\vec{p}_{2}=0 .{ }^{14}$ The relation between $E_{\text {lab }}$ and $\vec{p}^{2}$ reads (here and in what follows: $p \equiv|\vec{p}|)$ :

- Proton-proton case:

$$
p^{2}=\frac{1}{2} m_{p} E_{\mathrm{lab}}
$$

- Neutron-neutron case:

$$
p^{2}=\frac{1}{2} m_{n} E_{\mathrm{lab}}
$$

- Neutron-proton case:

$$
p^{2}=\frac{m_{p}^{2} E_{\mathrm{lab}}\left(E_{\mathrm{lab}}+2 m_{n}\right)}{\left(m_{n}+m_{p}\right)^{2}+2 E_{\mathrm{lab}} m_{p}} .
$$

The relativistic Schrödinger equation for two protons or two neutrons in the c.m. system reads

$$
\left[\left(2 \sqrt{p^{2}+m}-2 m\right)+V\right] \Psi=E \Psi
$$

where $m$ is the proton or neutron mass. For the neutron-proton system it takes the form

$$
\left[\left(\sqrt{p^{2}+m_{n}^{2}}+\sqrt{p^{2}+m_{p}^{2}}-m_{n}-m_{p}\right)+V\right] \Psi=E \Psi .
$$

The free Hamiltonian $H_{0}$ can be expressed in terms of the mass $m$ defined as

$$
m=\frac{2 m_{p} m_{n}}{m_{p}+m_{n}},
$$

in the following way

$$
H_{0}=\sqrt{p^{2}+m_{n}^{2}}+\sqrt{p^{2}+m_{p}^{2}}-m_{n}-m_{p} \simeq 2 \sqrt{p^{2}+m^{2}}-2 m,
$$

modulo terms which are proportional to $\left(m_{p}-m_{n}\right)^{2}$. Taking into account such terms goes beyond the accuracy of the present analysis. We will therefore use the approximate expression (A.7) in this work, which leads to the Schrödinger equation of the type (A.4).

\section{Appendix B. Partial wave decomposition of the $N N$ potential}

In this appendix we describe the partial wave decomposition of the two-nucleon potential. For that we first rewrite the potential $V$ in the form

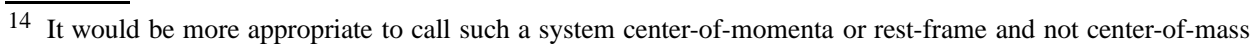
as usually done in the literature.
} 


$$
\begin{aligned}
V= & V_{C}+V_{\sigma} \vec{\sigma}_{1} \cdot \vec{\sigma}_{2}+V_{S L} i \frac{1}{2}\left(\vec{\sigma}_{1}+\vec{\sigma}_{2}\right) \cdot(\vec{k} \times \vec{q})+V_{\sigma L} \vec{\sigma}_{1} \cdot(\vec{q} \times \vec{k}) \vec{\sigma}_{2} \cdot(\vec{q} \times \vec{k}) \\
& +V_{\sigma q}\left(\vec{\sigma}_{1} \cdot \vec{q}\right)\left(\vec{\sigma}_{2} \cdot \vec{q}\right)+V_{\sigma k}\left(\vec{\sigma}_{1} \cdot \vec{k}\right)\left(\vec{\sigma}_{2} \cdot \vec{k}\right),
\end{aligned}
$$

with six functions $V_{C}\left(p, p^{\prime}, z\right), \ldots, V_{\sigma k}\left(p, p^{\prime}, z\right)$ depending on $p \equiv|\vec{p}|, p^{\prime} \equiv\left|\vec{p}^{\prime}\right|$ and the cosine of the angle between the two momenta is called $z$. These functions may depend on the isospin matrices $\tau$ as well. To perform the partial wave decomposition of $V$, i.e., to express it in the standard $l s j$ representation, we have followed the steps of Ref. [97]. In particular, we start from the helicity state representation $\left|\hat{p} \lambda_{1} \lambda_{2}\right\rangle$, where $\hat{p}=\vec{p} / p$ and $\lambda_{1}$ and $\lambda_{2}$ are the helicity quantum numbers corresponding to nucleons 1 and 2 , respectively. We then expressed the potential in the $\left|j m \lambda_{1} \lambda_{2}\right\rangle$ representation using the transformation matrix $\left\langle\hat{p} \lambda_{1} \lambda_{2} \mid j m \lambda_{1} \lambda_{2}\right\rangle$, given in Ref. [97]. The final step is to switch to the $|l s j\rangle$ representation. The corresponding transformation matrix $\left\langle l s j m \mid j m \lambda_{1} \lambda_{2}\right\rangle$ is given in Refs. [97,98].

For $j>0$, we obtain the following expressions for the nonvanishing matrix elements in the $|l s j\rangle$ representation:

$$
\begin{aligned}
& \langle j 0 j|V| j 0 j\rangle \\
& =2 \pi \int_{-1}^{1} d z\left\{V_{C}-3 V_{\sigma}+p^{\prime 2} p^{2}\left(z^{2}-1\right) V_{\sigma L}-q^{2} V_{\sigma q}-k^{2} V_{\sigma k}\right\} P_{j}(z), \\
& \langle j 1 j|V| j 1 j\rangle \\
& =2 \pi \int_{-1}^{1} d z\left\{\left[V_{C}+V_{\sigma}+2 p^{\prime} p z V_{S L}-p^{\prime 2} p^{2}\left(1+3 z^{2}\right) V_{\sigma L}\right.\right. \\
& \left.+4 k^{2} V_{\sigma q}+\frac{1}{4} q^{2} V_{\sigma k}\right] \\
& \times P_{j}(z)+\left[-p^{\prime} p V_{S L}+2 p^{\prime 2} p^{2} z V_{\sigma L}-2 p^{\prime} p\left(V_{\sigma q}-\frac{1}{4} V_{\sigma k}\right)\right] \\
& \left.\times\left(P_{j-1}(z)+P_{j+1}(z)\right)\right\}, \\
& \langle j \pm 1,1 j|V| j \pm 1,1 j\rangle \\
& =2 \pi \int_{-1}^{1} d z\left\{p^{\prime} p\left[-V_{S L} \pm \frac{2}{2 j+1}\left(-p^{\prime} p z V_{\sigma L}+V_{\sigma q}-\frac{1}{4} V_{\sigma k}\right)\right]\right. \\
& \times P_{j}(z)+\left[V_{C}+V_{\sigma}+p^{\prime} p z V_{S L}+p^{\prime 2} p^{2}\left(1-z^{2}\right) V_{\sigma L}\right. \\
& \left.\left. \pm \frac{1}{2 j+1}\left(2 p^{\prime 2} p^{2} V_{\sigma L}-\left(p^{\prime 2}+p^{2}\right)\left(V_{\sigma q}+\frac{1}{4} V_{\sigma k}\right)\right)\right] P_{j \pm 1}(z)\right\}, \\
& \langle j \pm 1,1 j|V| j \mp 1,1 j\rangle \\
& =\frac{\sqrt{j(j+1)}}{2 j+1} 2 \pi \int_{-1}^{1} d z\left\{-p^{\prime} p\left(4 V_{\sigma q}-V_{\sigma k}\right) P_{j}(z),\right.
\end{aligned}
$$




$$
\begin{aligned}
& +\left[\mp \frac{2 p^{\prime 2} p^{2}}{2 j+1} V_{\sigma L}+p^{\prime 2}\left(2 V_{\sigma q}+\frac{1}{2} V_{\sigma k}\right)\right] P_{j \mp 1}(z) \\
& \left.+\left[ \pm \frac{2 p^{\prime 2} p^{2}}{2 j+1} V_{\sigma L}+p^{2}\left(2 V_{\sigma q}+\frac{1}{2} V_{\sigma k}\right)\right] P_{j \pm 1}(z)\right\}
\end{aligned}
$$

Here, $P_{j}(z)$ are the conventional Legendre polynomials. For $j=0$ the two nonvanishing matrix elements are

$$
\begin{aligned}
\langle 000|V| 000\rangle= & 2 \pi \int_{-1}^{1} d z\left\{V_{C}-3 V_{\sigma}+p^{\prime 2} p^{2}\left(z^{2}-1\right) V_{\sigma L}-q^{2} V_{\sigma q}-k^{2} V_{\sigma k}\right\}, \\
\langle 110|V| 110\rangle= & 2 \pi \int_{-1}^{1} d z\left\{z V_{C}+z V_{\sigma}+p^{\prime} p\left(z^{2}-1\right) V_{S L}+p^{\prime 2} p^{2} z\left(1-z^{2}\right) V_{\sigma L}\right. \\
& \left.-\left(\left(p^{\prime 2}+p^{2}\right) z-2 p^{\prime} p\right) V_{\sigma q}-\frac{1}{4}\left(\left(p^{\prime 2}+p^{2}\right) z+2 p^{\prime} p\right) V_{\sigma k}\right\} .
\end{aligned}
$$

Note that sometimes another notation is used in which an additional overall minus sign enters the expressions for the off-diagonal matrix elements with $l=j+1, l^{\prime}=j-1$ and $l=j-1, l^{\prime}=j+1$.

\section{Appendix C. Momentum space treatment of the Coulomb interaction}

In this appendix we would like to explain our way of treating the nucleon-nucleon scattering problem in the presence of the Coulomb interaction in momentum space (following closely Ref. [7]). The starting point is the nonrelativistic Schrödinger equation of the form (3.7) or (3.12), where the potential consists of two pieces: the short-range one given by the strong interaction and the long-range one given by the Coulomb force.

As the Coulomb potential is of infinite range, the $S$-matrix has to be formulated in terms of asymptotic Coulomb states. Therefore, the phase shifts for a given angular momentum $l$ due to the strong potential in the presence of the long-range electromagnetic interactions, denoted by $\delta_{l}^{l}$, are defined in terms of a linear combination of (ir)regular Coulomb-functions $F(G)$ as

$$
\chi_{l}^{l}(r)=F_{l}(r)+\tan \left(\delta_{l}^{l}\right) G_{l}(r)
$$

analogously to the expression for an arbitrary potential of short range (i.e., in the absence of the Coulomb force)

$$
\chi_{l}^{s}(r)=F_{l}^{0}(r)+\tan \left(\delta_{l}^{s}\right) G_{l}^{0}(r)
$$

with $F^{0}, G^{0}$ denoting solutions of the Coulomb problem with zero charge (conventionally expressed in terms of Bessel and Neumann functions) and the corresponding phase shift is called $\delta_{l}^{s}$. So far, we have restricted ourselves to uncoupled channels. We will consider the coupled case later on. 
As Eq. (C.1) exhibits asymptotical Coulomb-states, we have to re-express our Lippmann-Schwinger equation in terms of them. A very convenient scheme for inclusion of the Coulomb force in momentum space was suggested long time ago by Vincent and Phatak [99] and is used in the present analysis. In what follows we will briefly describe this approach.

The starting point of this technique is the observation, that for a potential of the form

$$
V=V_{\mathrm{C}}+V_{\mathrm{S}}
$$

with

$$
V_{\mathrm{S}} \psi_{l}=0 \quad(r \geqslant R),
$$

and $\psi_{l}$ the two-nucleon wave-function for a given angular momentum, two exact solutions for the wave-function can be given for every point on a sphere with radius $R+\epsilon$. One is of the form as in Eq. (C.1), and another one according to Eq. (C.2) with the phase shifts calculated for the following potential as in Eq. (C.3), with $\mathrm{V}_{\mathrm{C}}$, however, being the Fouriertransformed Coulomb-potential integrated to the radius $R$,

$$
V_{\mathrm{C}}\left(\left|\vec{q}^{\prime}-\vec{q}\right|\right)=\int_{0}^{R} d^{3} r e^{i\left(\vec{q}^{\prime}-\vec{q}\right) \cdot \vec{r}} \frac{\alpha}{r}=\frac{4 \pi \alpha}{\left|\vec{q}^{\prime}-\vec{q}\right|^{2}}\left(1-\cos \left(\left|\vec{q}^{\prime}-\vec{q}\right| R\right)\right) .
$$

Here, $\vec{q}, \vec{q}^{\prime}$ are the cms momenta and $\alpha$ is the fine-structure constant. On the above-defined sphere, both wave functions describe the same system. Now we know how to obtain an expression for the strong phase shift $\delta_{l}^{l}$ in the presence of the Coulomb interaction in terms of the short-range shift $\delta_{l}^{s}$ in the absence of electromagnetism: we only have to match the two solutions. This is most conveniently done by requiring the logarithmic derivative of both solutions to be equal, what enables us to express the strong shift in the presence of the Coulomb force in a Wronskian form

$$
\tan \left(\delta_{l}^{l}\right)=\frac{\tan \left(\delta_{l}^{s}\right)\left[F, G_{0}\right]+\left[F, F_{0}\right]}{\left[F_{0}, G\right]+\tan \left(\delta_{l}^{s}\right)\left[G_{0}, G\right]}
$$

with

$$
[F, G]=\left(G \frac{d F}{d r}-F \frac{d G}{d r}\right)_{r=R} .
$$

Let us now extend the previous consideration to the coupled case. For that we replace Eq. (C.1) by the matrix equation

$$
\chi^{l}(r)=F(r)-m q K^{l} G(r),
$$

where $K^{l}$ is the $K$-matrix for the strong potential in the presence of the Coulomb interaction, $\chi^{l}(r)$ is the $2 \times 2$ matrix which contains the wave functions

$$
\chi^{l}(r)=\left(\begin{array}{ll}
\chi_{j-1, j-1}^{l}(r) & \chi_{j-1, j+1}^{l}(r) \\
\chi_{j+1, j-1}^{l}(r) & \chi_{j+1, j+1}^{l}(r)
\end{array}\right),
$$

and $F(r)$ and $G(r)$ are the $2 \times 2$ matrices which contain the Coulomb wave functions

$$
F(r)=\left(\begin{array}{cc}
F_{j-1}(r) & 0 \\
0 & F_{j+1}(r)
\end{array}\right), \quad G(r)=\left(\begin{array}{cc}
G_{j-1}(r) & 0 \\
0 & G_{j+1}(r)
\end{array}\right) .
$$


All subscripts in the above equations refer to the values of the angular momentum $l$. Analogously, Eq. (C.2) has to be replaced by

$$
\chi^{s}(r)=F^{0}(r)-m q K^{s} G^{0}(r),
$$

where $K^{s}$ is the $K$-matrix for the strong potential only.

Matching now the wave function $\chi^{l}(r)$ with $\chi^{s}(r)$, calculated from the potential $V$ defined in Eqs. (C.3) and (C.5), at some radius $R$ by equating the corresponding logarithmic derivatives as described above one obtains for the $K$-matrix $K^{l}$ :

$$
\begin{aligned}
K^{l}= & \frac{1}{m q}\left[F\left(F_{0}-m q K^{s} G_{0}\right)^{-1}\left(F_{0}^{\prime}-m q K^{s} G_{0}^{\prime}\right)-F^{\prime}\right] \\
& \times\left[G\left(F_{0}-m q K^{s} G_{0}\right)^{-1}\left(F_{0}^{\prime}-m q K^{s} G_{0}^{\prime}\right)-G^{\prime}\right]^{-1} .
\end{aligned}
$$

The only remaining difficulty is the determination of the matching radius $R$, because the given solution is wrong as long as (C.4) is not valid. On the other hand, it is not possible to extend $R$ to arbitrarily large values, because the cosine in Eq. (C.5) will cause rapid oscillations. $R \sim 10 \mathrm{fm}$ turns out to be a good choice, see [7]. We use the value $R=12 \mathrm{fm}$ in the present analysis.

\section{Appendix D. Effective range expansion}

In this appendix we collect the formulae for the $S$-wave effective range expansion. In the simplest case of the scattering with the finite-range potential, the quantity $k \cot \left(\delta_{0}\right)$, where $\delta_{0}$ is the $S$-wave phase shift and $k$ is the c.m.s. momentum, is well known to have the low-momentum (or effective range) expansion:

$$
k \cot \left(\delta_{0}\right)=-\frac{1}{a}+\frac{1}{2} r k^{2}+v_{2} k^{4}+v_{3} k^{6}+v_{4} k^{8}+\mathcal{O}\left(k^{10}\right) .
$$

Here $a$ is the scattering length, $r$ the effective range and $v_{2,3,4}$ the shape parameters.

In the presence of the long-range potential the effective range expansion has to be modified. In that case one usually defines an effective range function instead of the quantity $k \cot \left(\delta_{0}\right)$, in which the left-hand singularities due to the long-range interaction are removed, see Ref. [50] for more details. In the case of the modified Coulomb potential given in Eq. (2.53), the effective range function $F_{C}$ takes the form [50]

$$
F_{C}=C_{0}^{2}\left(\eta^{\prime}\right) k \cot \left(\delta_{0}^{C}\right)+2 k \eta^{\prime} h\left(\eta^{\prime}\right),
$$

where the quantity $\eta^{\prime}$ is given by

$$
\eta^{\prime}=\frac{m_{p}}{2 k} \alpha^{\prime}
$$

and the functions $C_{0}^{2}\left(\eta^{\prime}\right)$ (the Sommerfeld factor) and $h\left(\eta^{\prime}\right)$ read

$$
C_{0}^{2}\left(\eta^{\prime}\right)=\frac{2 \pi \eta^{\prime}}{e^{2 \pi \eta^{\prime}}-1} \quad \text { and } \quad h\left(\eta^{\prime}\right)=\operatorname{Re}\left[\Psi\left(1+i \eta^{\prime}\right)\right]-\ln \left(\eta^{\prime}\right) .
$$

Here, $\Psi$ denotes the digamma function. Notice that the phase shift $\delta_{0}^{C}$ is the $S$-wave phase shift of the finite-range plus Coulomb potential with respect to Coulomb wave functions. 
In the notation of Section $5, \delta_{0}^{C}$ should be written as $\delta_{\mathrm{C} 1+\mathrm{N}}^{\mathrm{C} 1}$. The effective range expansion for the function $F_{C}$ is

$$
F_{C}=-\frac{1}{a^{C}}+\frac{1}{2} r^{C} k^{2}+v_{2}^{C} k^{4}+v_{3}^{C} k^{6}+v_{4}^{C} k^{8}+\mathcal{O}\left(k^{10}\right) .
$$

In a general case of an arbitrary long-range interaction, the effective range function may be obtained, e.g., along the lines of Ref. [100] provided that the long-range potential is weak enough to be treated perturbatively. For more discussion on the effective range expansion in presence of electromagnetic interaction the reader is referred to [50].

\section{References}

[1] S. Weinberg, Nucl. Phys. B 363 (1991) 3.

[2] S.R. Beane, P.F. Bedaque, W.C. Haxton, D.R. Phillips, M.J. Savage, in: M. Shifman (Ed.), At the Frontier of Particle Physics, vol. 1, World Scientific, Singapore, 2001, pp. 133-269.

[3] P.F. Bedaque, U. van Kolck, Annu. Rev. Nucl. Part. Sci. 52 (2002) 339.

[4] E. Epelbaum, A. Nogga, W. Glöckle, H. Kamada, U.-G. Meißner, H. Witala, Phys. Rev. C 66 (2002) 064001.

[5] D.R. Entem, R. Machleidt, Phys. Rev. C 68 (2003) 041001.

[6] R. Machleidt, Some issues concerning the nucleon-nucleon interaction based upon chiral effective field theory, talk given at the INT program Theories of Nuclear Forces and Nuclear Systems, INT, Seattle, USA, September 29-December 5, 2003.

[7] M. Walzl, U.-G. Meißner, E. Epelbaum, Nucl. Phys. A 693 (2001) 663.

[8] S.R. Beane, P.F. Bedaque, M.J. Savage, U. van Kolck, Nucl. Phys. A 700 (2002) 377.

[9] S.R. Beane, M.J. Savage, Nucl. Phys. A 713 (2003) 148.

[10] E. Epelbaum, U.-G. Meißner, W. Glöckle, Nucl. Phys. A 714 (2003) 535.

[11] S.R. Beane, M.J. Savage, Nucl. Phys. A 717 (2003) 91.

[12] C. Ordóñez, L. Ray, U. van Kolck, Phys. Rev. C 53 (1996) 2086.

[13] J.L. Friar, S.A. Coon, Phys. Rev. C 49 (1994) 1272.

[14] N. Kaiser, R. Brockmann, W. Weise, Nucl. Phys. A 625 (1997) 758.

[15] E. Epelbaum, W. Glöckle, U.-G. Meißner, Nucl. Phys. A 637 (1998) 107.

[16] E. Epelbaum, W. Glöckle, U.-G. Meißner, Nucl. Phys. A 671 (2000) 295.

[17] E. Epelbaum, W. Glöckle, U.-G. Meißner, Eur. Phys. J. A 19 (2004) 125.

[18] N. Kaiser, Phys. Rev. C 64 (2001) 057001.

[19] N. Kaiser, Phys. Rev. C 61 (2000) 014003.

[20] N. Kaiser, Phys. Rev. C 62 (2000) 024001.

[21] E. Epelbaum, W. Glöckle, U.-G. Meißner, Eur. Phys. J. A 19 (2004) 401.

[22] E. Epelbaum, Doctoral thesis, published in: Berichte des Forschungszentrums Jülich, No. 3803 (2000).

[23] N. Fettes, U.-G. Meißner, S. Steininger, Nucl. Phys. A 640 (1998) 199.

[24] N. Kaiser, private communication.

[25] J.L. Friar, Phys. Rev. C 60 (1999) 034002.

[26] D.R. Entem, R. Machleidt, Phys. Rev. C 66 (2002) 014002.

[27] N. Kaiser, Phys. Rev. C 63 (2001) 044010.

[28] M.R. Robilotta, Phys. Rev. C 63 (2001) 044004.

[29] R. Higa, M.R. Robilotta, Phys. Rev. C 68 (2003) 024004.

[30] T. Becher, H. Leutwyler, Eur. Phys. J. C 9 (1999) 643.

[31] U. van Kolck, PhD thesis, University of Texas at Austin, UMI-94-01021-mc (1993).

[32] U. van Kolck, et al., Phys. Rev. Lett. 80 (1998) 4386.

[33] U. van Kolck, J.L. Friar, T. Goldman, Phys. Lett. B 371 (1996) 169.

[34] E. Epelbaum, U.-G. Meißner, Phys. Lett. B 461 (1999) 287.

[35] J.L. Friar, et al., Phys. Rev. C 68 (2003) 024003. 
[36] S.A. Coon, J.A. Niskanen, Phys. Rev. C 53 (1996) 1154.

[37] J.A. Niskanen, Phys. Rev. C 65 (2002) 037001.

[38] V.G.J. Stoks, et al., Phys. Rev. C 48 (1993) 792.

[39] R. Urech, Nucl. Phys. B 433 (1995) 234.

[40] U.-G. Meißner, S. Steininger, Phys. Lett. B 419 (1998) 403.

[41] G. Müller, U.-G. Meißner, Nucl. Phys. B 556 (1999) 265.

[42] N. Fettes, U.-G. Meißner, S. Steininger, Phys. Lett. B 451 (1999) 233.

[43] N. Fettes, U.-G. Meißner, Phys. Rev. C 63 (2001) 045201.

[44] N. Fettes, U.-G. Meißner, Nucl. Phys. A 693 (2001) 693.

[45] J. Gasser, M.A. Ivanov, E. Lipartia, M. Mojzis, A. Rusetsky, Eur. Phys. J. C 26 (2002) 13.

[46] J. Gasser, H. Leutwyler, Phys. Rep. 87 (1982) 77.

[47] V. Pascalutsa, D.R. Phillips, nucl-th/0308065.

[48] J. Friar, U. van Kolck, Phys. Rev. C 60 (1999) 034006.

[49] G.J.M. Ausin, J.J. de Swart, Phys. Rev. Lett. 50 (1983) 2039.

[50] J.R. Bergervoet, et al., Phys. Rev. C 38 (1988) 15.

[51] V.G. Stoks, J.J. de Swart, Phys. Rev. C 42 (1990) 1235.

[52] V.G. Stoks, PhD thesis, Nijmegen (1990).

[53] E.A. Ueling, Phys. Rev. 48 (1935) 55.

[54] L. Durand III, Phys. Rev. 108 (1957) 1597.

[55] D. Eiras, J. Soto, Phys. Lett. B 491 (2000) 101.

[56] H.P. Stapp, T.J. Ypsilantis, N. Metropolis, Phys. Rev. 105 (1957) 302.

[57] J.M. Blatt, L.C. Biedenharn, Phys. Rev. 86 (1952) 399;

J.M. Blatt, L.C. Biedenharn, Rev. Mod, Phys. 24 (1952) 258.

[58] H. Kamada, W. Glöckle, Phys. Rev. Lett. 80 (1998) 2547.

[59] J.L. Friar, Phys. Rev. C 20 (1979) 325.

[60] M. Kohno, J. Phys. G: Nucl. Phys. 9 (1983) L85.

[61] D.R. Phillips, T.D. Cohen, Nucl. Phys. A 668 (2000) 45.

[62] S. Klarsfeld, et al., Nucl. Phys. A 456 (1986) 373.

[63] J. Martorell, D.W.L. Sprung, D.C. Zheng, Phys. Rev. C 51 (1995) 1127.

[64] J.L. Friar, J. Martorell, D.W.L. Sprung, Phys. Rev. A 56 (1997) 4579.

[65] R. Rosenfelder, Phys. Lett. B 479 (2000) 381.

[66] K. Melnikov, T. van Ritbergen, Phys. Rev. Lett. 84 (2000) 1673.

[67] I. Sick, Phys. Lett. B 576 (2003) 62.

[68] S. Kopecky, et al., Phys. Rev. Lett. 74 (1995) 2427.

[69] B.K. Jennings, Phys. Rev. C 62 (2000) 027602.

[70] M. Garcon, J.W. Van Orden, Adv. Nucl. Phys. 26 (2001) 293.

[71] R. Gilman, F. Gross, J. Phys. G 28 (2002) R37.

[72] M.C.M. Rentmeester, R.G.E. Timmermans, J.J. de Swart, Phys. Rev. C 67 (2003) 044001.

[73] P. Büttiker, U.-G. Meißner, Nucl. Phys. A 668 (2000) 97.

[74] C.R. Howell, et al., Phys. Lett. B 444 (1998) 252.

[75] D.E. González Trotter, et al., Phys. Rev. Lett. 83 (1999) 3788.

[76] P. Lepage, nucl-th/9706029.

[77] P. Lepage, Tutorial: renormalizing the Schrödinger equation, Talk given at the INT program Effective Field Theories and Effective Interactions, INT, Seattle, USA, June 25-August 5, 2000.

[78] J. Gegelia, G. Japaridze, Phys. Lett. B 517 (2001) 476.

[79] J. Gegelia, S. Scherer, nucl-th/0403052.

[80] S.R. Beane, et al., Phys. Rev. A 64 (2001) 042103.

[81] E. Epelbaum, et al., Eur. Phys. J. A 15 (2002) 543.

[82] J.R. Bergervoet, et al., Phys. Rev. C 41 (1990) 1435.

[83] M.M. Nagels, T.A. Rijken, J.J. de Swart, Phys. Rev. D 17 (1978) 768.

[84] E.M. Henley, D.A. Miller, in: M. Rho, D. Wilkinson (Eds.), Mesons in Nuclei, North-Holland, 1979, p. 406.

[85] R.B. Wiringa, et al., Phys. Rev. C 51 (1995) 38.

[86] G.A. Miller, M.K. Nefkens, I. Slaus, Phys. Rep. 194 (1990) 1.

[87] D.E. Gonzales Trotter, et al., Phys. Rev. Lett. 83 (1999) 3788. 
[88] V. Huhn, et al., Phys. Rev. C 63 (2000) 014003.

[89] X. Kong, F. Ravndal, Nucl. Phys. A 665 (2000) 137.

[90] J. Gegelia, Eur. Phys. J. A 19 (2004) 355.

[91] J.D. Jackson, J.M. Blatt, Rev. Mod. Phys. 22 (1950) 77.

[92] I. Fachruddin, Ch. Elster, W. Glöckle, Nucl. Phys. A 689 (2001) 507c.

[93] J.J. de Swart, C.P.F. Terheggen, V.G.J. Stoks, nucl-th/9509032.

[94] J.-W. Chen, G. Rupak, M.J. Savage, Nucl. Phys. A 653 (1999) 386.

[95] J.-W. Chen, G. Rupak, M.J. Savage, Phys. Lett. B 464 (1999) 1.

[96] M. Walzl, U.-G. Meißner, Phys. Lett. B 513 (2001) 37.

[97] K. Erkelenz, R. Alzetta, K. Holinde, Nucl. Phys. A 176 (1971) 413.

[98] M. Jacob, G. Wick, Ann. Phys. 7 (1959) 404.

[99] C.M. Vincent, S.C. Phatak, Phys. Rev. C 10 (1974) 391

[100] O. Hinckelmann, L. Spruch, Phys. Rev. A 3 (1971) 2.

[101] M.C.M. Rentmeester, private communication, 1999.

[102] C. van der Leun, C. Alderlisten, Nucl. Phys. A 380 (1982) 261.

[103] D.M. Bishop, L.M. Cheung, Phys. Rev. A 20 (1979) 381.

[104] T.E.O. Ericson, M. Rosa-Clot, Nucl. Phys. A 405 (1983) 497.

[105] N.L. Rodning, L.D. Knutson, Phys. Rev. C 41 (1990) 898.

[106] SAID on-line program R.A. Arndt, et al., http://gwdac.phys.gwu.edu.

[107] Nijmegen NN on-line program, http://nn-online.sci.kun.nl.

[108] G. Fink, et al., Nucl. Phys. A 518 (1990) 561.

[109] J. Sromicki, et al., Phys. Rev. Lett. 57 (1986) 2359.

[110] J. Wilczynski, et al., Nucl. Phys. A 425 (1984) 458.

[111] T.C. Montgomery, et al., Phys. Rev. C 16 (1977) 499.

[112] D.H. Fitzgerald, et al., Phys. Rev. C 21 (1980) 1190.

[113] R. Garrett, et al., Phys. Rev. C 21 (1980) 1149.

[114] A. Langsford, et al., Nucl. Phys. 74 (1965) 241.

[115] J.L. Romero, et al., Phys. Rev. C 17 (1978) 468.

[116] T. Rönnqvist, et al., Phys. Rev. C 45 (1992) R496.

[117] G.H. Stafford, et al., Lett. Nuovo Cimento 5 (1957) 1589.

[118] T.C. Griffith, et al., Proc. Phys. Soc. London, Sect. A 71 (1958) 305.

[119] J. Rahm, et al., Phys. Rev. C 63 (2001) 044001.

[120] A.J. Bersbach, et al., Phys. Rev. D 13 (1976) 535.

[121] A.F. Kuckes, et al., Phys. Rev. 121 (1961) 1226. 